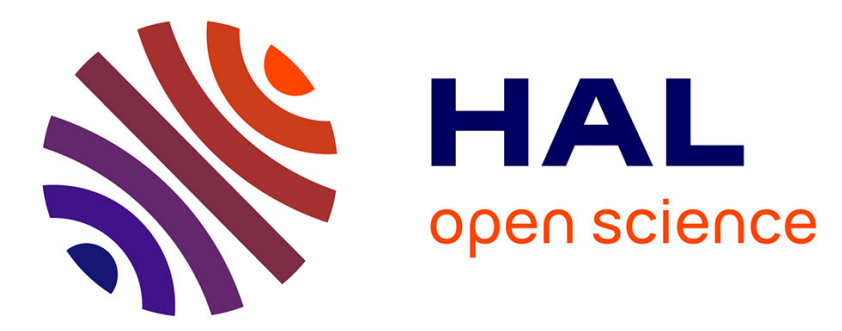

\title{
Homoparentalité et assistance médicale à la procréation : que sait-on du développement des enfants de mères lesbiennes ?
}

\author{
O. Vecho, Benoit Schneider, C. Zaouche-Gaudron
}

\section{- To cite this version:}

O. Vecho, Benoit Schneider, C. Zaouche-Gaudron. Homoparentalité et assistance médicale à la procréation : que sait-on du développement des enfants de mères lesbiennes ?. Neuropsychiatrie de l'Enfance et de l'Adolescence, 2018, 66 (6), pp.382-395. 10.1016/j.neurenf.2018.06.001 . hal02363480

\author{
HAL Id: hal-02363480 \\ https://hal.science/hal-02363480
}

Submitted on 17 Nov 2019

HAL is a multi-disciplinary open access archive for the deposit and dissemination of scientific research documents, whether they are published or not. The documents may come from teaching and research institutions in France or abroad, or from public or private research centers.
L'archive ouverte pluridisciplinaire HAL, est destinée au dépôt et à la diffusion de documents scientifiques de niveau recherche, publiés ou non, émanant des établissements d'enseignement et de recherche français ou étrangers, des laboratoires publics ou privés. 
NOTE : ce document est une version acceptée le 11/06/2018 par la revue Neuropsychiatrie de l'Enfance et de l'Adolescence.

\section{Référence :}

Vecho, O., Schneider, B., \& Zaouche-Gaudron, C. (2018). Homoparentalité et assistance médicale à la procréation : que sait-on du développement des enfants de mères lesbiennes ? Neuropsychiatrie de l'Enfance et de l'Adolescence. doi: 10.1016/j.neurenf.2018.06.001 
Homoparentalité et assistance médicale à la procréation : que sait-on du développement des enfants de mères lesbiennes ?

Same-sex parenting and assisted reproductive technology: what do we know about the development of children of lesbian mothers?

Vecho, O. ${ }^{\mathrm{a}}$, Schneider, B. ${ }^{\mathrm{b}}$ et Zaouche-Gaudron, C. ${ }^{\mathrm{c}}$

${ }^{a}$ Université Paris Nanterre, France, CliPsyD - EA 4430, ovecho@ parisnanterre.fr

${ }^{\mathrm{b}}$ Université de Lorraine, France, 2LPN - EA 7489, benoit.schneider@univ-lorraine.fr

${ }^{\mathrm{c}}$ Université Toulouse Jean Jaurès, France, UMR Lisst-Cers, chantal.zaouche @ univ-tlse2.fr

2 


\section{Homoparentalité et assistance médicale à la procréation : que sait-on du développement des enfants de mères lesbiennes ?}

\section{Résumé}

Buts de l'étude. La diversification des études relatives au développement des enfants de familles homoparentales, ainsi que les progrès méthodologiques dans leur conduite, permettent de mieux prendre en compte la spécificité des différentes méthodes disponibles aux couples de même sexe pour devenir parents. Le contexte créé par la révision des lois de bioéthique en France en 2018 invite à examiner de façon détaillée les recherches analysant spécifiquement le développement des enfants de mères lesbiennes conçus par assistance médicale à la procréation (AMP).

Méthode. Nous avons identifié et analysé 16 publications internationales (parues entre 1997 et 2017) concernant l'étude de 9 cohortes d'enfants de mères lesbiennes conçus par AMP. Cette analyse est complétée, en particulier, par l'examen de 24 publications visant les familles lesboparentales qui ne distinguent pas les situations de procréation avec ou sans assistance médicale (c'est-à-dire AMP ou auto-insémination non médicalisée du sperme d'un donneur connu), et nous avons enfin considéré ces résultats au regard des études sur les enfants adoptés par des couples de même sexe.

Résultats et conclusion. Les études révèlent essentiellement une homogénéité développementale entre enfants de mères lesbiennes et ceux de parents hétérosexuels (conçus naturellement ou par AMP), que l'on s'attache aux caractéristiques développementales des enfants ou aux relations familiales. Ces études analysent par ailleurs, de façon spécifique, les questions de l'accès aux informations relatives au donneur ainsi que de la stigmatisation dont ces familles peuvent faire l'objet.

Mots-clés : homoparentalité, mères lesbiennes, assistance médicale à la procréation, développement, enfant 


\title{
Same-sex parenting and assisted reproductive technology: what do we know about the development of children of lesbian mothers?
}

\begin{abstract}
Goals of the study. The diversification of studies about the development of children in samesex families, as well as the methodological advances in these studies, make it possible to better take into account the specificity of the different options available to same-sex couples to create a family. The revision of the bioethics laws in France in 2018 invites to examine in detail research specifically analyzing the development of children of lesbian mothers conceived by assisted reproductive technics (ART).
\end{abstract}

Method. We identified and analyzed 16 international publications (published between 1997 and 2017) concerning the study of 9 cohorts of children of lesbian mothers conceived by ART. We also supplemented this analysis with, in particular, the examination of 24 publications about planned lesbian families that do not distinguish between procreation situations with and without medical assistance (i.e., ART or self-insemination), and we also considered these results in relation to studies of children adopted by same-sex couples.

Results and conclusion. The studies essentially reveal a developmental homogeneity across children of lesbian mothers and those of heterosexual parents (conceived naturally or by ART), whether we focus on the developmental characteristics of children or on family relationships. These studies also specifically analyze the issues of access to donor information and the stigmatization that these families may face.

Keywords: same-sex parenting, lesbian mothers, assisted reproductive technics, development, children 


\section{Introduction}

\subsection{Le contexte créé par la révision des lois de bioéthique de 2011}

En France, la loi n 2011-814 du 7 juillet 2011 a chargé le Comité Consultatif National d'Éthique pour les sciences de la vie et de la santé (CCNE) d'organiser des États Généraux de la Bioéthique ${ }^{1}$. En vue d'une révision des lois de bioéthique, le CCNE était par cette voie invité à produire en 2018, à destination des pouvoirs publics, un rapport de synthèse sur 9 thèmes dont celui de l'Assistance Médicale à la Procréation (AMP). C'est donc un moment opportun pour proposer une contribution, qui s'inscrit dans un débat sociétal plus élargi, à la problématique de l'accès à la parentalité pour les couples lesbiens. En effet, les familles homoparentales ont désormais pris place dans l'espace des pluriparentalités qui rend compte des diversités croissantes des modes d'organisation de la vie privée et de l'articulation entre conjugalité et filiation. Un moment majeur a été, pour la France, la promulgation de la loi du 18 mai 2013 rendant accessible le mariage et l'adoption aux couples de même sexe. Cette évolution s'inscrit dans un mouvement très général qui concerne un grand nombre de pays, particulièrement les pays occidentaux (1). Les possibilités d'accès à la parentalité sont encadrées par des dispositions juridiques variables d'un pays à l'autre, et tolérées au plan collectif ou contournées au plan des stratégies personnelles. Une disposition générale du droit comme l'accès au mariage s'accompagne cependant parfois de nuances : les dispositions du code civil en France concernant le mariage des couples de sexes différents ont été transposées au bénéfice des couples de même sexe, mais à l'exception de quelques points importants, notamment celui concernant certaines des modalités de reconnaissance du lien de filiation ${ }^{2}$.

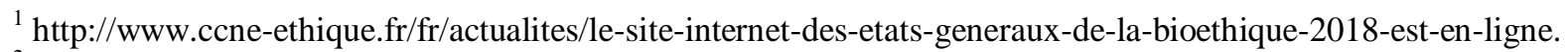

${ }^{2}$ L'adoption de l'enfant du conjoint au sein du mariage peut aujourd'hui se faire de la même manière pour les couples de sexes différents et les couples de même sexe, mais certaines procédures simples permettant rapidement et sans ambiguité la reconnaissance du lien de filiation (telles que la présomption de paternité ou la reconnaissance en mairie) restent réservées aux couples de sexes différents (2).
} 
Une revendication forte des femmes lesbiennes concerne le recours à l'AMP. Les techniques d'insémination artificielle avec donneur (IAD) ont été développées au cours des années 1970. En France, ce recours existait avant 1994 c'est-à-dire avant l'adoption de la première loi de bioéthique qui a restreint l'accès à l'AMP aux couples hétérosexuels, mais il concernait alors peu de couples (3). Les lois de bioéthique de 1994 (loi n²004-800 du 6 août 2004 relative à la bioéthique) et de 2004 (loi 2011-814 du 7 juillet 2011) ont défini les conditions actuelles d'accès à l'AMP : seuls les couples hétérosexuels vivant en couple et en âge de procréer, souffrant d'une pathologie de la fertilité ou risquant de transmettre une pathologie grave en procédant par eux-mêmes, peuvent recourir à ces techniques. Selon l'Agence de biomédecine (4), plus de 145000 tentatives d'AMP ont été réalisées en France en 2015 (toutes techniques confondues), conduisant à la naissance de près de 25000 enfants, soit 3,1\% des enfants nés la même année.

Les lois de bioéthique sont revues tous les 7 ans, mais la loi de 2011 n'a pratiquement rien changé aux dispositions retenues en 2004, en conséquence de quoi les femmes célibataires et les couples de femmes recourent aux services de pays limitrophes qui se montrent à cet égard plus ouverts (Belgique, Pays-Bas, Espagne...).

Une enquête par questionnaire menée en ligne en 2012 dans le cadre d'une étude sur le fonctionnement familial et conjugal des familles homoparentales a recueilli les réponses de 180 hommes gays et de 496 femmes lesbiennes vivant en couple et élevant un ou plusieurs enfants ou attendant l'arrivée de leur premier enfant $(5,6)$. Les auteurs ne prétendent pas à la représentativité de l'échantillon puisque sans données fiables sur la population de référence, mais ils rapportent que la moitié des enquêtés n'avaient jamais adhéré à une association de défense des droits des personnes homosexuelles. Les mères représentent $74 \%$ des répondants et, sur l'ensemble des enfants élevés par un couple lesbien, 51\% sont nés par IAD (c'est en particulier le cas pour les familles ayant les enfants les plus jeunes, et cette modalité de 
filiation semble s'imposer comme le moyen privilégié d'avoir des enfants pour les couples lesbiens). Les enfants conçus par IAD représentent donc dans cette enquête $38 \%$ des enfants c'est-à-dire plus du tiers de l'échantillon. Rappelons que l'Institut National d'Études Démographiques (INED), sur la base de statistiques allemandes et néerlandaises, estimait en 2006 qu'il existait en France entre 24000 et 40000 enfants élevés par un couple de même sexe (7) ; l'INSEE recensait, quant à elle, en 2013, 200000 personnes déclarant vivre en couple de même sexe (8) dont $10 \%$ déclaraient avoir au moins un enfant. Tout en restant prudent sur le passage d'une source de données à l'autre, mais en considérant que les pratiques se sont plutôt intensifiées depuis la recension de ces données chiffrées, on peut considérer que plusieurs milliers d'enfants en France, à l'heure actuelle, sont issus d'une IAD en contexte homoparental lesbien.

Le gouvernement français a choisi en 2013, suite à l'ouverture du mariage et de l'adoption aux couples de même sexe, de s'en remette au CCNE sur la question de l'ouverture de l'AMP aux couples de femmes. La doctrine des pouvoirs publics en France a été de longue date de définir l'AMP comme un recours médical et non « de convenance », comme une réponse médicale à un problème biologique et non à une demande sociale. Mais si la philosophie générale des lois de bioéthique a été de restreindre l'AMP à des cas relevant du «pathologique » et ainsi d'exclure tous les cas liés à l'évolution des formes de vie en couple et des familles, l'évolution des techniques de procréation a induit, de fait, de nouvelles attentes au sein des couples lesbiens (3). Dans son avis rendu public le 15 juin 2017 «sur les demandes sociétales de recours à l'AMP»(9), le CCNE, étayant sa réflexion à partir du contexte historique, juridique et international, et tenant compte des évolutions techniques ainsi que des changements dans l'organisation des relations humaines, s'est montré à la fois précautionneux et tout en prudente évolution, traversé qu'il était par les positions partagées en son sein. Dans son chapitre 3: «Réflexion sur les demandes d'assistance médicale à la 
procréation par des couples de femmes ou des femmes seules », il est conduit à conclure que «la majorité des membres du CCNE se prononcent pour la recommandation d'ouverture de l'AMP aux couples de femmes et aux femmes seules, sous réserve de la prise en compte de conditions d'accès et de faisabilité » (p. 28). D'abord, notons une évolution au regard des rappels précédents quant à la conception de la santé : il pointe que si «le législateur a consacré dans le droit de la bioéthique une vision strictement thérapeutique du rôle de la médecine », il peut être conduit à prendre en compte « la souffrance ressentie du fait d'une infécondité secondaire à des orientations personnelles. » (p. 23). Le CCNE fait ensuite part de ses questions visant la responsabilité du législateur quant aux conséquences d'un recours des couples de femmes à l'IAD concernant « les relations de l'enfant à son environnement familial » (p. 28) en mettant l'accent sur la question des origines, de l'absence de père et des repères familiaux. Mais toujours dans la partie générale du texte figure une partie spécifique faisant état des «positions divergentes au sein de CCNE» et un point concerne l'avis sur l'« accès des femmes à l'IAD ». Ses auteurs adoptent une «position recommandant le statu quo » (p. 49). Les justifications développées ( $c f$. partie «L'enfant le premiers tiers vulnérable ») apparaissent fondées sur un argumentaire peu développé qui mérite un débat contradictoire. L'annexe 5 de l'avis du CCNE propose un bilan synthétique relatif aux «Études sur le devenir des enfants dans les familles homoparentales et monoparentales » (p. 66). Il consiste essentiellement à mettre en exergue les réserves méthodologiques de certaines études disponibles pour justifier la position de prudence retenue. Ainsi, la sélection des recherches à l'appui du propos est plus que partielle, voire partiale. Pour exemples : la référence citée pour Gross (10) n'aborde en rien la question spécifique de l'AMP alors que celle mentionnée supra (5), et qui porte spécifiquement sur ce type de configuration familiale, n'est pas citée ; quant à la référence aux études de Regnerus $(11,12)$ qui ont donné lieu à une polémique scientifique de grande ampleur quant à ses conclusions sur le 
développement déficitaire des enfants de familles homoparentales, elle implique une lecture critique non retenue $\mathrm{ici}^{3}$. Le lecteur est au final renvoyé à des résultats de recherche à venir, par exemple l'étude «Homoparentalité, fonctionnement familial, développement et socialisation de l'enfant » $\mathrm{DEVHOM}^{4}$ : en France, en parallèle de l'Étude longitudinale Française depuis l'Enfance (ELFE) menée depuis 2011 et prévue pour ausculter le quotidien de 18000 familles pendant vingt ans, une étude de cohorte de familles homoparentales a en effet débuté en 2014 réunissant en particulier des chercheurs de l'INED et de l'INSERM pour apporter des connaissances sur la socialisation et le développement identitaire des enfants. Toutefois, les premiers résultats annoncés ne sont pas encore rendus publics.

Il est donc nécessaire de formuler de façon un peu plus précise les acquis de la recherche. En réalité, les acquis sont sans doute plus solides et documentés que ceux qui président à la synthèse du CCNE. Avant d'y revenir, il est utile de faire une rapide mention du contexte sociétal et scientifique dans lequel s'inscrit ce débat en France.

\subsection{L'évolution des attitudes et des opinions}

Plusieurs sondages menés en France depuis les années 1990 font apparaître une opinion publique de plus en plus favorable à l'ouverture de l'AMP aux couples lesbiens : $24 \%$ en 1990, 51\% en 2004, 60\% en 2017 (16). Ces changements rapides s'inscrivent dans une évolution plus générale des attitudes envers les droits accordés aux personnes homosexuelles puisque le même constat peut être fait concernant par exemple le mariage entre personnes de même sexe (17).

\footnotetext{
${ }^{3}$ L'étude de Regnerus a fait l'objet de vives critiques au plan méthodologique et de l'éthique de la recherche $(13,14)$ et une nouvelle analyse des données de cette étude en a invalidé les conclusions sur les effets délétères de l'homoparentalité (15).

${ }_{4} \mathrm{http}: / /$ www.agence-nationale-recherche.fr/informations/actualites/detail/devhom-premiere-etude-de-grandeampleur-sur-les-familles-homoparentales-en-france/.
} 
L’opinion en France a reflété le mouvement général vis-à-vis de l'homoparentalité en se montrant progressivement plus tolérante malgré les débats houleux au moment de l'adoption de la loi concernant le mariage entre personnes de même sexe. Si les «avis experts» ont longtemps été orientés par les tenants d'une psychanalyse 'normative', d'autres psychanalystes se sont montrés d'emblée plus ouverts, voire militants d'une approche appuyée sur une réflexion théorique et clinique argumentée $(18,19)$, qui s'interroge sur le développement des enfants de couples homosexuels en développant en particulier une déconstruction critique de l'appareil conceptuel de la psychanalyse mobilisé par certains pour s'opposer aux ouvertures législatives. Cette orientation initiale a fortement évolué comme l'atteste une pétition initiée en 2012 («Lettre ouverte des psychanalystes face à l'égalité des droits et au mariage pour tous ») $)^{5}$ soutenant «qu'il ne revient pas à la psychanalyse de se montrer moralisatrice et prédictive. Au contraire, rien dans le corpus théorique qui est le nôtre ne nous autorise à prédire le devenir des enfants quel que soit le couple qui les élève. La pratique psychanalytique nous enseigne depuis longtemps que l'on ne saurait tisser des relations de cause à effet entre un type d'organisation sociale ou familiale et une destinée psychique singulière. De plus, la clinique de nombre d'entre nous avec des enfants de couples "homosexuels" atteste que ce milieu parental n'est ni plus ni moins pathogène qu'un autre environnement. ».

Les «spécialistes» ou «experts »s'inscrivent de fait dans un mouvement général d'évolution, et on voit leurs propres réserves se lever progressivement (20-22) en même temps que les grilles de lectures théoriques sont elles-mêmes l'objet d'évolutions.

\footnotetext{
${ }^{5}$ http://petitionpublique.fr/PeticaoListaSignatarios.aspx?pi=P2012N30808.
} 


\subsection{Le développement des enfants élevés au sein des familles homoparentales}

L'approche dont nous nous revendiquons ici, complémentaire aux approches cliniques tout aussi nécessaires pour saisir la dynamique psychique des sujets, s'appuie sur les recherches «déployant une méthodologie d'évaluation explicite et apportant au moins des résultats quantitatifs traités statistiquement» et «impliquant au moins une évaluation des enfants » (23) (p. 281).

Les recherches relatives aux enfants issus d'une IAD et élevés en famille homoparentale s'inscrivent d'abord dans le cadre élargi des recherches sur les enfants élevés dans ce type de famille. Nous pouvons donc faire d'abord rappel de bilans généraux, et nous nous appuierons essentiellement pour ce faire sur la revue de littérature de deux d'entre nous, fréquemment citée, publiée en 2005 (23), bilan partiellement actualisé en 2012 (24). Les conclusions essentielles à tirer de ces bilans - et confortées par d'autres bilans du même type $(25,26)$ nous apparaissaient les suivantes :

- l'examen des études disponibles sur le développement des enfants élevés en contexte homoparental ne conduit pas au constat d'une plus grande vulnérabilité de ces enfants. Julien (25) (p. 372) indiquait : «Les résultats convergent tous vers un message clair et sans ambiguïté : lorsque les enfants de parents homosexuels ont des problèmes d'adaptation, d'autres facteurs que la simple orientation sexuelle des parents sont responsables de ces difficultés »;

- nous pointions un certain nombre de critiques méthodologiques classiquement mentionnées : taille des échantillons ou modalités de recrutement des sujets. Elles étaient souvent justifiées, exigeant précaution dans l'utilisation des résultats et leur généralisation possible, rendant aussi compte des difficultés à approcher ces situations familiales nouvelles. Mais nous faisions aussi le constat d'une rigueur méthodologique croissante. Pour exemple, le mode d'accès à la parentalité au sein des familles (par exemple adoption, 
recomposition homoparentale, IAD, etc.) était indisponible dans 4 recherches sur 11 (36\%) avant 1990, dans 4 recherches sur 24 (16\%) avant 2000, dans 3 recherches sur $35(8 \%)$ après 2000 ;

- en nous interrogeant sur «le développement de l'enfant», nous relevions une évolution des critères pris en compte pour en attester, traduisant de fait l'évolution du regard des chercheurs - donc indirectement celui de la société - sur l'homoparentalité, elle-même en transformation du fait de l'évolution des connaissances médicales et du droit, et de l'ouverture de la société à ces familles. Nous reviendrons dans la présente analyse sur le détail de ces critères d'observation.

Plus récemment, une revue de littérature spécifiquement consacrée aux enfants adoptés par des couples de même sexe conforte la littérature précédemment analysée : le contexte de l'adoption homoparentale n'apparaît pas plus «à risque » que celui de l'adoption hétéroparentale (27). En conclusion, nous écrivions : «La diversité des situations examinées par la prise en compte de ces familles, en enrichissant l'analyse des facteurs en jeu dans le processus adoptif, contribue secondairement à une meilleure compréhension de ce dernier. » (p. 412). Nous proposons donc ici de poursuivre le même type de démarche en ce qui concerne les familles de mères lesbiennes élevant des enfants conçus par IAD.

\subsection{Le développement des enfants de couples lesbiens conçus par IAD}

Que sait-on du développement des enfants de couples lesbiens conçus par IAD ? Les données de synthèse publiées jusqu'à ce jour sont limitées, et ce pour deux raisons. D’abord, et nous y reviendrons, leur nombre est encore restreint quoique tangible. Ensuite, parce que quelques synthèses sont entachées de défauts méthodologiques. Ces deux remarques préliminaires doivent être comprises comme s'inscrivant dans une évolution générale des études où la qualité méthodologique des recherches s'accroit progressivement. 
Il y a quelques années, Rozée (3) a proposé un premier bilan basé sur plusieurs recherches mais certaines d'entre elles ne figureront pas dans le bilan proposé ici parce que les sujets des études mentionnées $(28,29)$ ne relevaient pas explicitement ou strictement de l'AMP pour des couples de mères lesbiennes. Outre cette première référence, deux revues de questions ont déjà abordé le traitement des données disponibles sur le développement des enfants de couples lesbiens nés par IAD. Bos et Van Balen (30) proposent une analyse de 29 publications portant sur des recherches empiriques (publiées entre 1983 et 2010) visant les familles ayant eu recours à l'AMP (hétéroparentales, monoparentales, homoparentales), toutefois le mode de traitement des données ne permet pas d'isoler les résultats spécifiques aux enfants de mères lesbiennes conçus par IAD. Ilioi et Golombok (31) ont quant à elles centré leur analyse sur 17 publications visant uniquement les adolescents conçus par IAD et leur adaptation psychologique, dont 6 (publiées entre 2005 et 2012) concernent les familles homoparentales.

\section{Méthodologie}

Le premier auteur mène depuis le début des années 2000 une veille documentaire concernant les publications scientifiques internationales relatives à l'homoparentalité en sciences humaines et sociales, à partir des bases de données Cairn, PsycArticles, Psychology and Behavioral Sciences Collection, Psycinfo, Pubmed, ScienceDirect et SocIndex. Elle a permis d'identifier au $1^{\text {er }}$ janvier 2018 plus de 1000 publications dans ce domaine parues depuis 1972 .

Pour la présente analyse, seules les publications permettant d'isoler des résultats spécifiques aux enfants conçus par AMP et issus du projet parental de mères lesbiennes (seules ou en couple) ont été retenues, sans critère de période de publication. Ainsi, nous n'avons pas retenu les publications où les échantillons incluaient des enfants conçus par AMP 
lorsque ces derniers étaient confondus avec des enfants adoptés par des couples de même sexe ou nés d'une union hétérosexuelle antérieure et qu'il n'était pas possible d'en extraire des résultats spécifiques aux enfants qui intéressent cette revue de littérature. Au-delà du corpus de plus de 1000 publications déjà disponibles, nous avons examiné les bibliographies des articles retenus pour notre analyse et conduit une recherche bibliographique complémentaire spécifique aux situations d'AMP dans les bases de données citées plus haut et à partir des formules suivantes ou leur traduction en anglais : «insémination + mères lesbiennes », «assistance médicale à la procréation + mères lesbiennes », «procréation médicalement assistée + mères lesbiennes ». Nous avons ainsi identifié 16 publications issues de 9 recherches correspondant à 9 cohortes d'enfants. Le Tableau I présente les caractéristiques des études ainsi que les dimensions du développement de l'enfant évaluées à chaque recueil de données. Les 9 recherches y sont ordonnées par ordre chronologique de leur première publication et sont numérotées de R1 à R9.

La première publication de la recherche R2 (32-34) date de 1995, celle de la recherche R4 (35) de 2003, et la recherche R6 (36) a donné lieu à une première publication en 2010, mais aucune des publications antérieures à celles retenues ici ne concerne directement le développement des enfants. La publication R3 (37) est issue d'une étude longitudinale menée aux Pays-Bas par Bos depuis les années 2000, elle a donné lieu à 5 autres publications concernant le développement des enfants, toutefois ces dernières ne fournissent pas de résultats propres aux enfants de l'échantillon conçus par AMP qui sont confondus avec des enfants conçus par auto-insémination (sans aide médicale). Pour la même raison, nous n'avons pas retenu pour notre analyse les nombreuses publications issues de l'étude longitudinale menée par Gartrell aux États-Unis depuis les années 1990. Ces deux études longitudinales contribuent toutefois de façon majeure aux connaissances sur le développement des enfants nés du projet de mères lesbiennes et élevés en familles homoparentales, le plus 
souvent sans père, notamment sur les questions de sexualité, d'identité et comportements de genre ou encore de stigmatisation. Enfin, nous n'avons pas retenu la recherche de Baiocco et ses collègues, à nouveau par manque de distinction entre enfant conçus par AMP (représentant la majorité de l'échantillon selon les auteurs) et enfants non conçus par AMP. Toutefois, le lecteur trouvera leurs caractéristiques principales et leurs résultats en Annexe 1 ( $c f$. Tableau III), et nous examinerons si elles confortent ou non les résultats des 16 publications retenues et comment elles peuvent les compléter le cas échéant.

Le Tableau II ( $c f$. Annexe 1) présente les caractéristiques détaillées des 16 publications retenues en mentionnant les périodes de recueil des données, les caractéristiques des échantillons d'enfants de mères lesbiennes, celles des enfants des éventuels groupes de comparaison, les outils utilisés pour recueillir les données, et les résultats qui concernent au moins les enfants (par souci de simplification et pour répondre plus précisément à notre objectif, les résultats relatifs aux parents qui ne sont pas mis en lien avec ceux des enfants ne sont pas reportés). Certaines informations indisponibles dans les publications nous ont été communiquées par les auteurs, elles sont alors suivies d'un astérisque (*).

\section{Résultats de l'analyse des publications}

Comme indiqué dans le Tableau I, les 9 études ont produit 16 publications sur le développement des enfants qui, elles-mêmes, reposent sur un total de 11 recueils de données différents (3 recueils de données successifs ont été réalisés pour la recherche R1 (38-42)). Selon les indicateurs présentés, les résultats seront rapportés au nombre d'études retenues (Nét $=9)$, de publications $(\mathrm{Npub}=16)$, ou de recueils de données réalisés $(\mathrm{Nrd}=11)$.

Les 16 publications retenues sont parues entre 1997 et 2017, et leurs échantillons proviennent des États-Unis pour 6 études, d'Europe pour 2 études et d'Israël pour 1 étude.

\subsection{Caractéristiques des populations}


Les 9 recherches ont porté sur des échantillons de 12 à 283 enfants de mères lesbiennes. Selon les études, ces enfants sont, soit tous issus d'un donneur anonyme (R1, R3, R7, R8 : (37-44)), soit tous issus d'un donneur dont l'identité sera accessible à la majorité (R4, R9 : $(35,45))$. Cette information n'est pas disponible pour les autres études (R2, R5, R6 : (32$34,36,46,47))$.

$\mathrm{Au}$ moment des recueils de données, la majorité des échantillons étaient constitués uniquement d'enfants élevés par un couple lesbien (R1, R2, R3, R4, R5, R6, R9 : (32-42,4547)). Les autres échantillons étaient à la fois constitués d'enfants élevés par un couple lesbien et d'enfants élevés par une mère lesbienne seule : ces deux sous-groupes étaient soit différenciés (R2, R7 : (32-34,43)) soit non différenciés (R6, R8 : $(36,44))$ dans les traitements statistiques.

Concernant le genre des enfants, les informations disponibles pour 8 recueils de données montrent une représentation plus importante de garçons (57\%) que de filles (46\%). De plus, dans $50 \%$ des publications $(33,34,37-39,41,43,45)$, la variable «genre de l'enfant » est soit utilisée comme variable indépendante, soit contrôlée statistiquement dans les analyses ou au préalable. Cette variable est par ailleurs peu prise en compte dans les études qui délivrent des résultats fondés sur des statistiques descriptives.

Enfin, du point de l'âge des enfants, les travaux reposent sur des échantillons assez homogènes. Ainsi, parmi les 9 études, 4 concernent uniquement des enfants (R1 à R3, R7 : (32-34,37-43)), 2 uniquement des adolescents (R4, R8: $(35,44))$, et 1 implique uniquement des adultes (R9: (45)), 1 des adolescents et des adultes (R5:(46,47)) et 1 à la fois des enfants, des adolescents et des adultes (R6: (36)). 


\subsection{Caractéristiques méthodologiques des études}

Nous retiendrons ici trois critères méthodologiques: le mode de recrutement, les méthodes de recueil des données utilisées par les chercheurs et le recours à des groupes témoins.

On peut repérer trois modes de recrutement parmi les 9 études. D'abord, 5 d'entre elles ont sollicité des participants auprès de centres médicaux d'AMP (R1 à R4, R9 : (32-34,3742,45)). En marge des centres médicaux, une organisation non gouvernementale et non lucrative (Donor Sibling Registry, DSR) a été créée en 2000 aux États-Unis afin d'aider les enfants conçus par AMP à retrouver leur donneur et/ou les autres enfants issus de ce donneur en proposant un site internet de référencement des individus (à partir des numéros d'identification uniques donnés aux donneurs dans leur pays). Trois études ont ainsi diffusé leurs annonces recherche auprès des personnes inscrites au DSR (R5, R6, R8 : $(36,44,46,47))$. Enfin, deux études ont eu recours à des modes de recrutement plus classiques tels que des annonces dans la presse ou sur des forums internet, dans des centres de loisirs, des associations de soutien à la parentalité $(\mathrm{R} 3, \mathrm{R} 7:(37,43))$.

Concernant le choix des méthodes de recueil des données, il apparaît que 7 des 11 recueils de données s'appuient sur des entretiens menés avec les enfants $(64 \%, \mathrm{R} 1 / 2, \mathrm{R} 1 / 3, \mathrm{R} 4, \mathrm{R} 5 / 1$, R6, R8, R9 : (35,36,39,40,44-46)), 5 sur des questionnaires remplis par les enfants (45\%, R1/1, R1/2, R1/3, R7, R8 : (38-40,43,44)), 5 sur des questionnaires renseignés par les parents (45\%, R1/1, R1/2, R1/3, R2/1, R7 : (32,38-40,43)), 3 sur des questionnaires remplis par les enseignants des enfants $(27 \%, \mathrm{R} 1 / 1, \mathrm{R} 1 / 3, \mathrm{R} 2 / 1:(32,38,40))$ et 2 sur des entretiens menés avec les parents $(18 \%, \mathrm{R} 1 / 2, \mathrm{R} 1 / 3:(39,40))$.

La question des groupes témoins doit être envisagée du point de vue des publications et non des études, car pour une même étude on peut trouver des publications avec groupe témoin 
ou sans. Ainsi, 5 des 16 publications (31\%) ne comparent l'échantillon avec aucun groupe témoin (R1/2, R1/5, R7, R8, R9 : $(39,42-45))$, ce sont le plus souvent les études qui présentent des résultats descriptifs. Les 11 autres publications (69\%) présentent des comparaisons avec un ou des groupe(s) témoin(s) constitué(s) spécifiquement constitués pour l'étude. Les enfants de mères lesbiennes sont comparés avec des enfants de parents hétérosexuels conçus par AMP (8 publications, 73\%, R2/1, R2/2, R2/3, R4, R6 : (32$36,46,47)$ ) ou conçus naturellement (2 publications, 18\%, R1/3, R1/4 : $(40,41)$ ), avec simultanément 2 groupes d'enfants de parents hétérosexuels conçus par AMP ou naturellement (1 publication, 9\%, R1/1 : (38)), ou encore avec un groupe d'enfants de mères lesbiennes conçus par auto-insémination (non médicale) (1 publication, 9\%, R3 : (37)).

Qu'en est-il de la qualité de l'appariement entre les groupes? Parmi les 11 publications avec groupe(s) témoin(s), 3 ne permettent pas d'évaluer la qualité de l'appariement avec le groupe témoin faute d'informations détaillées (R5/1, R5/2, R6 : (36,46,47)). Les résultats qui suivent ne concernent donc que les 8 autres publications. Concernant l'âge des enfants, toutes les publications font apparaître un appariement adéquat. Dans 7 d'entre elles, les groupes sont bien appariés du point de vue de la répartition garçons / filles, avec de faibles différences de proportion, et dans 1 publication la proportion de garçons est plus élevée de $18 \%$ dans le groupe témoin (R1 : (38-42)) par rapport au groupe d'enfants de mères lesbiennes. Les 8 publications présentent également des échantillons bien appariés du point de vue du statut de singleton ou de membre d'une fratrie (le groupe d'enfants de mères lesbiennes et le groupe témoin sont tous deux soit constitués uniquement de singletons soit constitués de singletons et d'enfants en fratrie). Du point de vue du rang de naissance des enfants, 6 publications présentent un appariement correct (5 avec uniquement des aînés (R1, R2/1 à R3 : (32-34,3742)), 1 avec à la fois des aînés et des cadets dans les deux groupes (R4 : (35)) et 2 ne délivrent pas cette information $(\mathrm{R} 1 / 3, \mathrm{R} 1 / 4:(40,41))$. 
Sur l'ensemble des 11 publications avec groupe(s) témoin(s), 9 des publications avec groupe(s) témoin(s) (82\%) présentent un appariement correct dans le sens où le groupe d'enfants de mères est lesbiennes est au moins comparé à un groupe d'enfants de couples hétérosexuels (R1, R2, R4, R5 : (32-35,38,40,41,46,47)) ou lesbiens (R3 : (37)), et dans 1 publication (9\%) les informations disponibles ne permettent pas d'évaluer cette caractéristique de l'appariement (R6 : (36)).

\subsection{Thèmes abordés et principaux résultats}

Pour l'analyse des résultats, nous avons réalisé une synthèse des données essentielles issues des 9 recherches qui correspondaient à nos critères de sélection. En considérant l'ensemble des thématiques abordées, des critères d'observation et des outils retenus par les chercheurs, nous pouvons regrouper ces résultats en trois catégories de données essentielles, à savoir les données relatives au développement psychologique des enfants ${ }^{6}$ (notamment, comportements, adaptation sociale et émotions), aux relations familiales, et à la connaissance/non connaissance du donneur.

\subsubsection{Les données relatives au développement psychologique des enfants}

Ces données portent toutes sur les groupes de sujets âgés de 3 à 10 ans.

\section{- Les problèmes comportementaux et émotionnels}

Quatre études (R1, R2, R3, R7 : $(32,33,37,38,40,42,43))$ ont eu recours au Child Behaviour Checklist (CBCL), rempli par les parents et mesurant notamment les problèmes internalisés (émotionnels) et externalisés (comportementaux). Aucune d'entre elles ne relève de différence de problèmes comportementaux entre les enfants de mères lesbiennes et ceux des groupes de comparaison ou de la population de référence de l'outil. La recherche R2

\footnotetext{
${ }^{6}$ Le terme enfant est utilisé ici en termes de niveau générationnel et non d'âge, ainsi lorsque les études ont porté sur des adultes, il y sera fait référence en tant qu' « enfants » de couples de même sexe.
} 
$(32,33)$ confirme ces constats en ayant recours au Teacher's Report Form (TRF ; version du CBCL remplie par les enseignants) alors que la recherche R1 (40) conclut que les enseignants perçoivent davantage de problèmes totaux (externalisés et internalisés additionnés) et d'attention chez les enfants de mères lesbiennes que chez ceux du groupe de comparaison. La recherche R1 a également utilisé le Youth Self-Report (YSR; version du CBCL remplie par les enfants eux-mêmes) qui montre moins de comportements agressifs chez les enfants de mères lesbiennes que chez ceux du groupe de comparaison (40).

La recherche R7 (43) relève quant à elle moins de comportements antisociaux et de sentiment de solitude chez les enfants de mères lesbiennes que chez ceux de parents hétérosexuels (seuls ou en couple) (Children's Peer Relation Scale, CPRS, rempli par les enfants).

\section{- Les comportements genrés}

La recherche R1 (38) ne relève pas de différence de comportements genrés entre enfants de mères lesbiennes et ceux des groupes de comparaison (Preschoolers' Activities Inventory, PSAI, rempli par les parents).

- L'estime de soi et les compétences sociales

La recherche R1 $(40,42)$ n'observe pas de différence d'estime de soi globale, de sentiment d'acceptation sociale, de sentiment de compétences scolaires, physiques, sportives, de comportements et de relations avec les pairs, entre enfants de couples lesbiens et ceux du groupe de comparaison (Perceived Competence Scale for Children, PCSC, rempli par les enfants).

La recherche R7 (43) ne mentionne pas de différence entre enfants de mères lesbiennes et ceux de parents hétérosexuels (seuls ou en couple) concernant les sentiments de compétences 
cognitives, physiques, dans la relation à la mère et dans les relations avec les pairs (Pictorial Scale of Perceived Competence and Social Acceptance, PSPCSA, rempli par les enfants).

- Conclusion

Le type d'observation faisant apparaitre une meilleure adaptation comportementale ou sociale d'enfants élevés dans le cadre de familles homoparentale a déjà été relevé dans certaines études (27). Il doit être utilisé avec prudence dans la mesure où les écarts entre les évaluations restent modestes et que, dans l'ensemble, les résultats sont parfois en faveur ou en défaveur de chacune des configurations familiales. D'une part il s'agit d'être attentifs aux effets de sélection des familles comme dans le cadre de l'adoption, qui pourrait conduire, du fait de leur nouveauté et originalité, a un effet de sélectivité plus accru des couples homoparentaux ; d'autre part on peut envisager une attention plus accrue au plan éducatif de la part de familles exposées à un environnement plus complexe et de ce fait plus vigilantes à sensibiliser leurs enfants à des stratégies d'adaptation psycho-sociales. Au final ces observations invitent à être attentifs aux dynamiques familiales et à la spécificité de leur fonctionnement interne au-delà de leur configuration (cf. infra).

Mais le constat général est donc bien au final celui, en conformité avec les bilans précédents visant les familles homoparentales, d'une quasi absence d'effet de la configuration familiale sur les critères développementaux retenus.

\subsubsection{Les données relatives aux relations familiales}

La recherche $\mathrm{R} 1$ ne repère pas de différences de sentiments positifs et négatifs envers les parents entre enfants de mères lesbiennes et ceux des groupes de comparaison (Family Relations Test, FRT, rempli par les enfants) (38); pas de différence de qualité des relations parent-enfant (comportements et sentiments) entre les familles de mères lesbiennes et le groupe de comparaison (Parent-Child Interaction Questionnaire, rempli par les enfants) (41); 
pas de différence de qualité entre les interactions mère biologique-enfant dans les familles de mères lesbiennes et les interactions mères-enfant dans les deux groupes de comparaison (Quality of the Parent-Child Relationship, QPCR, rempli par les parents) (38). Elle mentionne cependant que la qualité des interactions mère non biologique-enfant dans les familles de mères lesbiennes est plus élevée que celle des interactions père-enfant dans les deux groupes de comparaison (38).

Dans la recherche R2, les auteurs se sont intéressés aux liens entre, d'une part, la satisfaction parentale dans la répartition des tâches quotidiennes et des prises de décision pour la famille (Who Does What, WDW) et, d'autre part, les problèmes comportementaux de l'enfant (TRF) (32) : pour tous les groupes, plus les parents non-biologiques sont satisfaits de la répartition des tâches ménagères, plus les scores de problèmes externalisés des enfants sont faibles. Pour les enfants de couples lesbiens plus précisément, plus les mères biologiques sont satisfaites de la répartition des tâches ménagères, moins elles rapportent de problèmes externalisés; plus les mères non-biologiques sont satisfaites de la répartition des tâches ménagères et des prises de décision liées à la famille, moins elles rapportent de problèmes externalisés ; plus les mères non-biologiques participent aux tâches liées à l'enfant, moins les mères biologiques rapportent de problèmes externalisés. Par ailleurs, plus les interactions parent-enfant sont décrites comme dysfonctionnelles par les parents (Parenting Stress IndexShort Form, PSI-SF) et plus leur stress parental est élevé (PSI-SF), plus les enfants ont de problèmes externalisés (CBCL, TRF) (33). Les scores de problèmes des enfants n'apparaissent pas liés aux dimensions parentales de dépression (Center for Epidemiologic Studies Depression scale, CES-D), d'ajustement conjugal (Lock-Wallace Marital Adjustment Test, LWMAT ; Partnership questionnaire, PQ) et d'estime de soi (Rosenberg Self-Esteem scale, RSE) (33). 
Au final, il apparaît là encore que la configuration familiale se révèle en tant que telle une variable faiblement explicative, mais on peut toutefois relever une tendance à une plus grande flexibilité et une meilleure communication au sein des familles homoparentales. Par ailleurs, c'est la qualité des relations au sein du couple parental indépendamment de sa configuration qui apparaît être en lien avec la manifestation de problèmes comportementaux et d'adaptation sociale chez l'enfant.

\subsubsection{Les données relatives à la connaissance/non connaissance du donneur}

En matière d'accès aux origines, les législations internationales relatives à l'AMP divergent : certains pays imposent l'anonymat, d'autres l'interdisent, et d'autres mettent à disposition des enfants, à leur majorité, des informations permettant d'identifier le donneur si celui-ci s'y est déclaré favorable.

Nous distinguerons ici les données relatives aux enfants jeunes et celles qui portent sur les adolescents et les adultes. Les premières essaient d'évaluer le lien entre certaines caractéristiques comportementales et l'information disponible chez les enfants concernant l'identité du donneur, les secondes s'attachent davantage aux modalités de la connaissance de sa situation par l'enfant et de la recherche d'information concernant le donneur.

- Les données sur les enfants jeunes

Dans la recherche R1 $(39,42)$, parmi tous les enfants conçus par AMP, 54\% préferent que le donneur soit anonyme, $27 \%$ souhaitent connaître son identité et $19 \%$ souhaitent des informations non identifiantes (physiques ou de personnalité), mais on n'observe pas de différence d'estime de soi (PCSC), de qualité des relations parents-enfants (Parent-Child Interaction Questionnaire - Child, PACHIQ-Ch, rempli par les enfants) ou de problèmes comportementaux (CBCL) entre enfants qui souhaitent avoir des informations sur l'identité du donneur et ceux qui ne le souhaitent pas. 
La recherche R3 (37) compare 52 enfants conçus par IAD (donneur anonyme) à 48 enfants conçus par auto-insémination du sperme d'un donneur connu (un ami des mères), tous de mères lesbiennes, et elle ne met pas en évidence de différence de problèmes de comportements internalisés, externalisés ou totaux (CBCL) entre les deux groupes. Toutefois, les enfants avec donneur connu ont des scores de problèmes sociaux plus élevés que ceux avec donneur anonyme, mais il n'y a pas de différence sur les autres échelles.

\section{- Les données spécifiques aux adolescents}

Dans la recherche R4 (35), 75,9\% des sujets ont le sentiment d'avoir toujours su qu'ils étaient nés par AMP, les autres se souviennent de l'avoir appris entre 4 et 9,5 ans, sans différence entre les 3 groupes constitués d'enfants de couples lesbiens, d'enfants de couples hétérosexuels conçus par AMP et d'enfants de mères seules conçus par AMP. Pour chacun des 3 groupes, apprendre et connaître l'origine de leur naissance n'a pas affecté, ou alors positivement, la relation des jeunes avec leur mère biologique, et n'a pas affecté leur relation avec leur père ou leur mère non-biologique.

La plupart des enfants - sans différence entre les trois groupes - se sentent à l'aise avec le fait d'avoir été conçus par AMP, quelques-uns sont neutres, surtout parce qu'ils estiment que cela n'affecte pas leur vie (44\%), mais aussi parce qu'ils se sentent uniques et très aimés au sein de leur famille $(40 \%)$.

Les enfants indiquent qu'ils ont modérément ou peu parlé de leur conception autour d'eux et, lorsqu'ils l'ont fait, c'est le plus souvent avec des membres de la famille étendue, un peu moins avec leurs amis et peu avec leurs enseignants. Les réactions de leur entourage sont évaluées comme plutôt positives, davantage par les enfants de couples lesbiens et de mères seules que par ceux de couples hétérosexuels. 
En moyenne, les sentiments des enfants envers le donneur sont plutôt positifs, et davantage encore chez les enfants de mères seules que chez ceux de couples lesbiens. Aucun enfant n'a décrit le donneur idéal comme un homme qui souhaiterait jouer le rôle de père auprès de lui ; 82,87\% des enfants s'interrogent sur la personnalité du donneur, mais seuls certains enfants de mères seules souhaitent connaître les motivations du donneur qui ont présidé au don. $\mathrm{Au}$ moment de la délivrance des informations relatives au donneur, en complément de son identité, les enfants souhaiteraient surtout avoir sa photo $(96,6 \%)$ ou connâitre sa situation familiale actuelle $(89,7 \%)$, mais $89,7 \%$ des enfants se disent de « modérément » à «très intéressés » d'avoir des contacts avec d'éventuels autres enfants issus du donneur et $85,7 \%$ de les rencontrer. Les enfants supposent que leurs parents réagiraient de façon plutôt positive à leur demande d'information ; 41,4\% des enfants projettent de contacter le donneur à leurs 18 ans, 20,7\% à 18 ans ou plus tardivement, et 27,6\% ne sont pas sûrs de le faire.

Dans la recherche R8 (44) (petit échantillon sans groupe comparatif), 10,5\% des enfants déclarent avoir toujours connu leur mode de conception, $47,4 \%$ ne se rappellent pas quand ils l'ont appris et $42,1 \%$ l'ont appris au plus tard à 7 ans. Aucun enfant n'a de contact avec son donneur, mais tous ont localisé au moins un autre enfant conçu à partir d'un de ses dons. Trente-sept pourcents des enfants présentent un pattern d'attachement insécure ; plus le score d'attachement sécure est élevé, plus le score de curiosité envers le donneur l'est aussi (Donor Conception Identity Questionnaire, DCIQ ; Friends and Family Interview, FFI) ; plus le score d'attachement insécure-désorganisé est élevé, plus le score d'évitement des questions liées au mode de conception l'est aussi. 
- Les données sur les adolescents et les adultes

Dans la recherche R4 (35), les enfants de couples lesbiens (36,5\%) et ceux de mères seules $(58,1 \%)$ ont davantage entamé de démarches pour obtenir des informations sur leur donneur que ceux de couples hétérosexuels $(23,3 \%)$.

Dans la recherche R5 (46,47), 56\% des enfants de couples lesbiens disent avoir toujours connu leur mode de conception, contre $45 \%$ de ceux de mères seules et $40 \%$ de ceux de couples hétérosexuels ; 63\% des enfants de couples hétérosexuels ont recherché leur donneur, contre $20 \%$ de ceux de mères seules et $13 \%$ de ceux de couples lesbiens ; $57 \%$ des enfants de couples hétérosexuels ont recherché les autres progénitures de leur donneur, contre $25 \%$ de ceux de mères seules et $14 \%$ de ceux de couples lesbiens. On ne note pas de différence de réaction quant à la recherche des autres progénitures du donneur ou du donneur lui-même entre les mères des 3 groupes (couples lesbiens, couples hétérosexuels, mères seules) ni entre les mères non-biologiques des couples lesbiens et les pères des couples hétérosexuels, mais ces réactions ne peuvent s'observer qu'à la condition de recherches effectives qui n'est pas la même dans chacun des groupes.

Dans la recherche R6 (36), 46\% des enfants de parents hétérosexuels et $79 \%$ de ceux de mères lesbiennes déclarent qu'ils ont toujours su avoir été conçus par AMP ; 24\% des enfants de parents hétérosexuels et $2 \%$ de ceux de mères lesbiennes déclarent avoir été informés de leur mode de conception après leurs 18 ans. À l'annonce de leur mode de conception, 25\% des enfants de parents hétérosexuels et $10 \%$ de ceux de mères lesbiennes déclarent avoir ressenti de la confusion ; 59\% des enfants de parents hétérosexuels et $78 \%$ de ceux de mères lesbiennes déclarent avoir eu du soutien de la part de leur mère biologique quand ils ont exprimé leur curiosité vis-à-vis du donneuralors que $20 \%$ des enfants de parents hétérosexuels et $60 \%$ de ceux de mères lesbiennes déclarent avoir eu du soutien de la part de leur père ou de leur mère non-biologique. 


\section{- Conclusion}

En conclusion, on ne note pas de lien spécifique chez les plus jeunes entre l'accès à l'information relative aux donneurs et les problèmes de comportements. Les adolescents de familles homoparentales semblent informés plus précocement de leur statut que ceux des autres familles. Cette connaissance n'affecte pas en tant que telle leurs relations avec leurs parents mais, d'une part, ils semblent peu troublés par cette annonce et, d'autre part, plus ils sont informés jeunes et plus ils semblent à l'aise pour rechercher l'information. Les enfants de familles homoparentales semblent également être plus soutenus pour le faire et ils informent plus volontiers leur entourage familial de leurs démarches. En outre, la curiosité semble être plus marquée chez les enfants des mères seules que chez ceux des couples lesbiens.

\section{Apport des recherches sur l'adoption par les couples de même sexe et sur les}

\section{«planned lesbian families »}

En conclusion à notre revue de littérature sur le développement des enfants adoptés par des couples de même sexe (27), nous constations que les recherches portant sur l'adoption confirmaient et amplifiaient les résultats des études antérieures qui portaient sur d'autres modes de construction des familles homoparentales au sens où elles ne mettaient pas à jour de difficultés spécifiques de développement chez les enfants concernés, en apportant des résultats d'autant plus nuancés que les différences peuvent parfois se révéler en faveur des enfants élevés en contexte homoparental. Nous avons vu que ces observations peuvent trouver un écho et une confirmation concernant les enfants conçus par IAD.

Nous relevions, par ailleurs, que les thématiques et les critères développementaux retenus confirmaient la tendance évolutive que nous avions observée au sujet d'autres configurations familiales : une moindre centration sur les questions d'identité sexuée ou de développement intellectuel et un intérêt plus marqué pour la qualité des liens sociaux et des relations 
d'attachement, même si le recours aux indicateurs de «problèmes comportementaux » ou « émotionnels » restaient fréquents du fait de leur simplicité d'usage et, pour certains d'entre eux, de leur adaptation à un large empan d'âge.

On peut noter ici qu'à certains égards les travaux portant sur l'IAD ne sont pas caractérisées par une ouverture aussi nette : on retrouve fréquemment examinés les problèmes d'adaptation comportementale (avec des outils comme le CBCL) et la prise en compte d'une série de variables relatives aux enfants. Mais l'étude des variables relatives au fonctionnement familial se révèle moins riche, tout en confirmant les résultats précédents, à savoir que les effets de la qualité des liens conjugaux et parentaux apparaissent davantage explicatifs du développement de l'enfant que ne l'est la configuration familiale.

En réalité, cette remarque doit être nuancée dans la mesure où un corpus de données est disponible dans la seconde partie des recherches présentées au Tableau III ( $c f$. Annexe 1) qui se réfèrent aux situations qui ne distinguent pas, au sein des couples de mère lesbiennes, les situation de recours à l'AMP et celles de recours à un donneur connu du couple en vue d'une auto-insémination non médicalisée. Dans la littérature, ces deux situations sont souvent réunies sous l'appellation de planned lesbian families (familles lesbiennes fondées sur un projet de couple). Ces recherches repérées au cours de notre recensement sont celles menées par H. Bos aux Pays-Bas, par N. Gartrell aux États-Unis et par R. Baiocco en Italie, elles constituent au total 24 publications (par la suite, respectivement dénommées RC1, RC2, qui sont des études longitudinales, et RC3).

En prenant en compte les deux sources données (adoption et familles lesbiennes planifiées), quatre thématiques méritent discussion.

\section{1. À propos de données confirmatoires}


Ces travaux confortent la tendance générale repérée précédemment concernant les problèmes comportementaux et émotionnels ( $\mathrm{RC} 1, \mathrm{RC} 2$ ) puisqu'il n'apparaît pas de différences notables avec les groupes témoins ou la population de référence.

En complément, la recherche RC3 (48) ne relève pas de différence du point de vue du fonctionnement émotionnel (labilité, régulation, symptômes) ou des problèmes comportementaux entre les enfants de couples lesbiens et ceux de couples gays et hétérosexuels. La publication RC1/5 (49) ne relève pas de différence d'estime de soi globale et de sentiment de compétences sociales entre enfants de mères lesbiennes et ceux du groupe de comparaison. Dans la publication RC1/6 (50), on observe même que les enfants de mères lesbiennes ont une estime de soi plus élevée et moins de problèmes de conduite que ceux du groupe de comparaison (qu'il s'agisse des filles ou des garçons).

\subsection{Discrimination et facteurs protecteurs}

Cette question aurait pu être abordée dans les recherches spécifiques à l'IAD, mais elle n'est traitée que dans les recherches qui ne distinguent pas les situations d'AMP et d'autoinsémination.

Des recherches constatent différentes formes de discrimination. La publication RC1/3 (51) indique que $61 \%$ des enfants ont été la cible de plaisanteries sur leur situation familiale, 57\% de questions gênantes, $45 \%$ d'expressions blessantes pour parler de l'orientation sexuelle de leurs mères, $31 \%$ de médisance, $26 \%$ d'exclusion, $21 \%$ de remarques désapprobatrices. Les publications RC2/4 (52) et RC2/16 (53) font apparaître que 41\% des sujets déclarent avoir fait l'expérience de l'homophobie (sans différence entre filles et garçons); en RC2/9 (54), 46\% des filles et 35,9\% des garçons déclarent avoir été victimes d'homophobie ; en RC2/14 (55), $50 \%$ des enfants ont fait l'expérience de l'homophobie du fait de leur configuration familiale, les filles davantage que les garçons. La stigmatisation provient surtout des pairs (essentiellement dans le contexte scolaire, sous forme d'exclusion du groupe, de rejet ou 
d'attitudes visant à les ridiculiser), parfois des membres de la famille étendue. Ces expériences de stigmatisation peuvent dépendre du contexte puisque la publication $\mathrm{RC} 1 / 4$ (56) mentionne que les garçons (15\%) et les filles $(22 \%)$ de l'échantillon néerlandais rapportent moins d'expériences homophobes que les garçons (37\%) et filles $(46 \%)$ de l'échantillon américain.

Les études indiquent aussi un lien récurent entre l'expérience de la discrimination ou du rejet perçus et les problèmes comportementaux et émotionnels. Selon la publication RC1/1 (57), plus les mères ont fait l'expérience du rejet en tant que mères lesbiennes au sein de leur environnement social, plus les niveaux de problèmes de comportements internalisés, externalisés et totaux chez les enfants sont élevés. En RC1/3 (51), les auteurs observent également que chez les garçons plus le niveau de stigmatisation est élevé, plus celui d'hyperactivité est élevé. D'après la publication RC2/1 (58), 69\% des enfants qui rapportent des expériences d'homophobie se sont sentis en colère, bouleversés ou tristes. En RC2/2 (59), $\mathrm{RC} 2 / 4$ (52), $\mathrm{RC} 2 / 14$ (55)et $\mathrm{RC} 2 / 16$ (53), les auteurs rapportent que les enfants qui mentionnent avoir fait l'expérience de l'homophobie ont des niveaux d'anxiété/dépression, de problèmes de relations sociales, d'attention, de délinquance, de comportements agressifs, internalisés et externalisés plus élevés que les autres enfants.

Enfin, les recherches font part de diverses stratégies de lutte ou de coping face à la discrimination et leurs effets. La recherche RC2 montre d'abord l'importance du soutien parental. En RC2/4 (52), 36\% des enfants déclarent que leurs mères les ont aidés à anticiper l'homophobie (pas de différence entre filles et garçons) et, en RC2/16 (53), parmi les enfants qui ont fait l'expérience de l'homophobie, ceux qui évaluent positivement leurs relations avec leurs parents et ceux qui se sentent intégrés dans leur groupe de pairs ont moins de problèmes psychologiques que les autres et sont plus satisfaits de leur vie que les autres. Ce soutien est étayé par l'environnement social et amical et le contexte pédagogique (RC2/2, (59)) : les liens 
entre expérience de l'homophobie et comportements délinquants sont moins forts chez les enfants dont les mères participent à la communauté lesbienne que chez les autres enfants ; le fait d'appartenir à une école qui délivre des enseignements sur les minorités sexuelles paraît également réduire les effets de l'homophobie sur les compétences sociales et les comportements agressifs des enfants. Les stratégies de coping observées peuvent être adaptatives et/ou non adaptatives (RC2/14, (55)) : 64\% des enfants disent avoir utilisé des stratégies de coping adaptatives (mise à distance des incidents, confrontation aux personnes qui les stigmatisent, recherche de soutien dans l'environnement social), 56\% des stratégies non adaptatives (non divulgation de la situation familiale, ignorer les incidents, éviter les personnes concernées).

\subsection{Information relative au donneur}

Ce thème est largement abordé dans les recherches centrées sur l'IAD, ce qui s'explique en particulier par le fait que les institutions qui procèdent aux inséminations ont à définir des procédures spécifiques relatives à la divulgation de l'information qui vont elles-mêmes dépendre du cadre législatif du pays dans laquelle l'IAD a lieu. Les publications RC2/5 (28) et $\mathrm{RC} 2 / 6$ (60) ne révèlent pas de différence de comportements internalisés, externalisés ou de compétences sociales selon que les enfants ont ou pourront avoir accès à l'identité du donneur et ceux qui ne le pourront pas.

\subsection{Dynamique familiale}

Le contexte familial a déjà été pris en compte dans la partie supra relative aux situations de discrimination en relavant les effets bénéfiques de l'accompagnement parental. Il s'agit plutôt ici de souligner que de nombreuses recherches, tel que nous l'avons montré dans le contexte de l'adoption, cherchent désormais à prendre en compte, comme facteur du développement, non plus tant la configuration familiale que ses modalités de fonctionnement en privilégiant les approches systémiques. De ce point de vue, la recherche RC3 (48) 
n'observe pas de différence entre les familles de mères lesbiennes et celles de pères gays (en termes de cohésion, flexibilité, désengagement, enchevêtrement, rigidité, chaos, communication, satisfaction) mais les familles de mères lesbiennes et de pères gays apparaissent plus flexibles et ont davantage recours à la communication dans leur fonctionnement que les familles de couples hétérosexuels.

La publication $\mathrm{RC} 1 / 2$ (57) met à jour de son côté les liens entre comportement parental, fonctionnement du couple et caractéristiques comportementales chez l'enfant. Alors que les auteurs ne relevaient pas de différence de problèmes de comportements internalisés et externalisés entre enfants de couples lesbiens et ceux de couples hétérosexuels, il s'avère que plus les parents sont satisfaits de l'implication parentale de leur partenaire et sont soutenus par lui/elle, moins les enfants ont de problèmes internalisés ; par ailleurs, plus les parents sont satisfaits de l'implication parentale de leur partenaire, moins les enfants ont de problèmes externalisés et totaux ; enfin, plus les parents sont perçus comme contrôlants, plus l'estime de soi des enfants est faible.

En conclusion, on note bien un ensemble de données convergentes relatives au développement des enfants conçus par IAD / auto-insémination non médicalisée ou adoption, mais on voit bien aussi que chaque contexte de recherche, tout en considérant qu'on ne peut pas tout étudier, sélectionne ses variables. Les études évoluent du fait d'un regard en renouvellement sur ces familles, en fonction de l'âge des enfants qui constituent les échantillons mais aussi en fonction des cadres de réflexion propres à chaque contexte : ainsi, les recherches centrées sur l'IAD s'attachent plus spécifiquement à la question des donneurs, celles sur l'adoption sont plus sensibles à un contexte global d'accueil. 


\section{Conclusion}

Le recours aux études relatives à l'homoparentalité dans les débats scientifiques et publics, qui eux-mêmes s'inscrivent dans un contexte culturel et moral plus général, amène classiquement à en critiquer certains aspects méthodologiques. Notre analyse des 16 publications sur l'AMP, confortée par celle des 24 publications complémentaires incluant les situations d'auto-insémination non médicalisée, révèle que les échantillons sont homogènes du point de vue de l'âge des enfants, plutôt de taille moyenne mais parfois plus conséquents, et que les résultats reposent le plus souvent sur des outils robustes éprouvés dans d'autres champs d'étude (CBCL par exemple). Certes, ces échantillons sont non probabilistes puisqu'ils ne sont pas constitués au gré de sollicitations de la population faites au hasard, mais il faut à ce titre rappeler que les enfants de couples hétérosexuels conçus par AMP constituent eux-mêmes une population peu fréquente (en France, 3\% des enfants nés par an). Dans ce contexte, le recours à un échantillonnage probabiliste des couples lesbiens ayant eu recours à l'AMP serait peu réaliste et obérerait toute tentative de mener des études. Au sujet des méthodes de recrutement qui sont classiquement critiquées, soulignons aussi qu'ici le recours aux réseaux «Lesbiennes, gays, bisexuels et transgenres »(LGBT) (associations, forums, etc.), pour autant que cela puisse constituer une limite majeure, reste ici marginal. Par ailleurs, lorsque les informations sont suffisamment détaillées, le recours aux groupes témoins apparaît dans l'ensemble pertinent, notamment au regard de leur nature (couples hétérosexuels ayant conçu naturellement ou par AMP) et de leurs caractéristiques (taille de l'échantillon, âge et genre des enfants, appartenance ou non à une fratrie, rang de naissance, statut conjugal des parents). Enfin, du point de vue du recueil de données, le plus souvent les enfants sont directement sollicités (sauf les plus jeunes), puis ce sont les parents et parfois les enseignants, ce qui permet parfois de confronter les points de vue. 
Le regard scientifique porté sur le développement des enfants de mères lesbiennes conçus par AMP doit aussi appeler à élargir les critères d'inclusion retenus ici pour examiner une littérature complémentaire, certes peu abondante pour le moment, concernant plus spécifiquement les mères elles-mêmes, au travers de leur santé psychologique, des processus qui sous-tendent le choix de celle qui portera l'enfant, de leur ajustement conjugal, du partage des tâches quotidiennes, ou encore de leur engagement dans la recherche du donneur qui a contribué à leur projet d'enfant. L'examen des études qualitatives menées auprès des enfants, le plus souvent autour des questions relatives au donneur, pourra également éclairer les processus développementaux et amener des nuances au plan clinique.

Nous pouvons aussi ajouter que, quelle que soit la configuration familiale (homo-, hétéroparentale ou femmes seules), l'AMP a induit une évolution majeure dans les modes de procréation déliée de la sexualité, et un «bouleversement des repères qu'elles [les aides médicales] introduisent au sein de la filiation » (61), puisqu'elle ne s'appuie plus seulement sur la filiation biologique et/ou génétique, mais aussi sur la filiation instituée et narcissique comme le propose Guyotat (62). Ainsi, chaque enfant devra composer avec un roman familial plus complexe qu'il devra construire à partir de plusieurs parents biologiques, géniteurs, sociaux, mais aussi à partir d'une narration la plus complète possible (63).

Du point de vue du développement des enfants, les études disponibles à ce jour n'amènent pas d'éléments qui justifieraient d'interdire l'accès de l'AMP aux couples lesbiens. Au-delà de la comparaison aux familles hétéroparentales prises pour référence, elles invitent à étendre l'analyse des processus intrafamiliaux et des effets de l'environnement social, notamment au regard de la question de l'anonymat du donneur et de la place qu'il « occupe » dans les familles homoparentales (y compris dans les familles hétéroparentales d'ailleurs), et de celle 
des effets de la stigmatisation dont les familles, et plus singulièrement les enfants, peuvent faire l'expérience dans les sociétés qui ouvrent l'AMP aux couples de même sexe.

\section{Références}

1. Carroll A, Mendos LR. State-Sponsored Homophobia 2017: A world survey of sexual orientation laws: criminalisation, protection and recognition. International Lesbian Gay Bisexual Trans and Intersex Association [Internet]. 2017. Available from: http://ilga.org/downloads/2017/ILGA_State_Sponsored_Homophobia_2017_WEB.pdf

2. Picardat M-C. Homoparentalité et PMA au regard de l'APGL. Psychol Psychol. 2017;252-253:34-8.

3. Rozée V. L'homomaternité issue de l'assistance médicale à la procréation en France : état des lieux d'un recours transnational. Raison-Publique [Internet]. 2012; Available from: http://www.raison-publique.fr/article531.html

4. Agence de biomédecine. Activité d'Assistance Médicale à la Procréation 2015 [Internet]. 2016. Available from: https://www.agence-

biomedecine.fr/annexes/bilan2016/donnees/procreation/01-amp/synthese.htm

5. Gross M, Courduriès J, de Federico A. Le recours à l'AMP dans les familles homoparentales : état des lieux. Résultats d'une enquête menée en 2012. Socio-Logos [Internet]. 2014;9. Available from: http:// journals.openedition.org/socio-logos/2870

6. Gross M, Courduriès J, de Federico A. Morphologie des familles homoparentales en France en 2012. In: Homosexualité et parenté. Paris: Dunod; p. 205-2012.

7. Festy P. Homoparentalités. Approches scientifiques et politiques. In: Cadoret A, Gross M, Mécary C, Perreau B, editors. Le recensement des familles homoparentales. Paris: PUF; 2006. p. 109-16.

8. Buisson G, Lapinte A. Le couple dans tous ses états. Non co-habitation, conjoints de même sexe, Pacs.... INSEE Prem [Internet]. 2013;1435. Available from: https://www.insee.fr/fr/statistiques/1281436

9. Comité Consultatif National d'Éthique pour les sciences de la vie et de la santé. Avis du CCNE sur les demandes sociétales de recours à l'assistance médicale à la procréation [Internet]. 2017. Available from: http://www.ccne-ethique.fr/fr/publications/avis-du-ccnedu-15-juin-2017-sur-les-demandes-societales-de-recours-lassistance\#.Wo6pga7iaUk

10. Gross M. L'homoparentalité et la transparentalité au prisme des sciences sociales : révolution ou pluralisation des formes de parenté ? Enfances Fam Génér [Internet]. 23. Available from: http://journals.openedition.org/efg/287

11. Regnerus M. How different are the adult children of parents who have same-sex relationships? Findings from the New Family Structures Study. Soc Sci Res. 2012;41(4):752-70. 
12. Regnerus M. Parental same-sex relationships, family instability, and subsequent life outcomes for adult children: Answering critics of the new family structures study with additional analyses. Soc Sci Res. 2012;41(6):1367-77.

13. Anderson E. The Need to Review Peer Review: The Regnerus Scandal as a Call to Action. J Gay Lesbian Ment Health. 2013;17(3):337-51.

14. Vecho $\mathrm{O}$, Schneider B. Homoparentalité et développement de l'enfant : réponse à Maurice Berger. Le Débat. 2015;183(1):150-6.

15. Cheng S, Powell B. Measurement, methods, and divergent patterns: Reassessing the effects of same-sex parents. Soc Sci Res. 2015;52:615-26.

16. Besmond de Sennevile L. PMA, GPA, fin de vie... la vague de fond libérale. LaCroix. 2018 Jan 3;

17. IFOP. Les Français et les droits des couples homosexuels deux ans après la 1ère manifestation de la Manif pour Tous [Internet]. 2014. Available from: http://www.ifop.com/?option=com_publication\&type=poll\&id=2839

18. Roudinesco E. La famille en désordre. Paris: Fayard; 2002.

19. Delaisi de Parceval G. Famille à tout prix. Paris: Seuil; 2008.

20. Vecho O, Schneider B. Attitudes des psychologues français à l'égard de l'homoparentalité. Psychiatr Enfant. 2012;55(1):269-92.

21. Vecho O, Schneider B. Attitudes envers l'homoparentalité: Une comparaison entre psychologues français et québécois. = Attitudes toward gay and lesbian parents: A comparison among French and Quebec psychologists. Can J Behav Sci Rev Can Sci Comport. 2015;47(1):102-12.

22. Zaouche-Gaudron C, Vecho O. Homoparentalité en questions. Andrologie. 2005;XV(3):287-94.

23. Vecho O, Schneider B. Homoparentalité et développement de l'enfant : bilan de trente ans de publications. Psychiatr Enfant. 2005;XLVIII(1):271-328.

24. Schneider B, Vecho O. Homoparentalité et développement de l'enfant : bilan de 40 ans de publications [Internet]. Colloque "Les rencontres nationales adoption et homoparentalité" organisé par le Conseil Général de l'Essonne; 2012 Apr 6; Evry. Available from: http://www.essonne.fr/fileadmin/divers/doc_adoption/Actes_Rencontres_Adoption_Hom oparentalite.pdf

25. Julien D. Trois générations de recherches empiriques sur les mères lesbiennes, les pères gais et leurs enfants. In: L'union civile Nouveaux modèles de conjugalité et de parentalité au 21ème siècle. Cowanswille: Edition von Blais; 2003. p. 359-84.

26. Patterson C. Children of lesbian and gay parents. Curr Dir Psychol Sci. 2006;15(5):241-4.

27. Schneider B, Vecho O. Le développement des enfants adoptés par des familles homoparentales: Une revue de la littérature. $=$ Development of children in same-sex 
adoptive families: A review of research. Neuropsychiatr Enfance Adolesc. 2015;63(6):401-12.

28. Gartrell N, Bos HMW. US National Longitudinal Lesbian Family Study: Psychological Adjustment of 17-Year-Old Adolescents. Pediatrics. 2010;126(1):28-36.

29. Golombok S, Badger S. Children raised in mother-headed families from infancy: a follow-up of children of lesbian and single heterosexual mothers, at early adulthood. Hum Reprod. 2010;25(1):150-7.

30. Bos H, van Balen F. Children of the new reproductive technologies: Social and genetic parenthood. Patient Educ Couns. 2010;81(3):429-35.

31. Ilioi EC, Golombok S. Psychological adjustment in adolescents conceived by assisted reproduction techniques: a systematic review. Hum Reprod Update. 2014;21(1):84-96.

32. Chan RW, Brooks RC, Raboy B, Patterson CJ. Division labor among lesbian and heterosexual parents : associations with children's adjustment. J Fam Psychol. 1998;12(3):402-19.

33. Chan RW, Raboy B, Patterson CJ. Psychosocial adjustment among children conceived by lesbian and heterosexual mothers. Child Dev. 1998;69(2):443-57.

34. Fulcher M, Chan R, Raboy B, Patterson C. Contact with grandparents among children conceived via donor insemination by lesbian and heterosexual mothers. Parent Sci Pract. 2002;2(1):61-76.

35. Scheib JE, Riordan M, Rubin S. Adolescents with open-identity sperm donors: reports from 12-17 year olds. Hum Reprod. 2005;20(1):239-52.

36. Beeson DR, Jennings PK, Kramer W. Offspring searching for their sperm donors: how family type shapes the process. Hum Reprod. 2011;26(9):2415-24.

37. Bos HMW, Hakvoort EM. Child adjustment and parenting in planned lesbian families with known and as-yet unknown donors. J Psychosom Obstet Gynecol. 2007;28(2):121-9.

38. Brewaeys A, Golombok S, Ponjaert I, Van Hall EV. Donor insemination : child development and family functioning in lesbian mother families. Hum Reprod Update. 1997;12(6):1349-59.

39. Vanfraussen K, Ponjaert-Kristoffersen I, Brewaeys A. An attempt to reconstruct children's donor concept : a comparison between children's and lesbian parents' attitudes towards donor anonymity. Hum Reprod Update. 2001;16(9):2019-25.

40. Vanfraussen K, Ponjaert-Kristoffersen I, Brewaeys A. What does it mean for youngsters to grow up in a lesbian family created by means of donor insemination? J Reprod Infant Psychol. 2002;20(4):237-54.

41. Vanfraussen K, Ponjaert-Kristoffersen I, Brewaeys A. Family functioning in lesbian families created by donor insemination. Am J Orthopsychiatry. 2003;73(1):78-90. 
42. Vanfraussen K, Ponjaert-Kristoffersen I, Brewaeys A. Why do children want to know more about the donor? The experience of youngsters raised in lesbian families. J Psychosom Obstet Gynaecol. 2003;24(1):31-8.

43. Shechner T, Slone M, Lobel TE, Shechter R. Children's adjustment in non-traditional families in Israel: the effect of parental sexual orientation and the number of parents on children's development. Child Care Health Dev. 2013;39(2):178-84.

44. Slutsky J, Jadva V, Freeman T, Persaud S, Steele M, Steele H, et al. Integrating donor conception into identity development: adolescents in fatherless families. Fertil Steril. 2016;106(1):202-8.

45. Scheib JE, Ruby A, Benward J. Who requests their sperm donor's identity? The first ten years of information releases to adults with open-identity donors. Fertil Steril. 2017;107(2):483-93.

46. Jadva V, Freeman T, Kramer W, Golombok S. The experiences of adolescents and adults conceived by sperm donation: comparisons by age of disclosure and family type. Hum Reprod. 2009;24(8):1909-1909.

47. Jadva V, Freeman T, Kramer W, Golombok S. Experiences of offspring searching for and contacting their donor siblings and donor. Reprod Biomed Online Reprod Healthc Ltd. 2010;20(4):523-32.

48. Baiocco R, Santamaria F, Ioverno S, Fontanesi L, Baumgartner E, Laghi F, et al. Lesbian mother families and gay father families in Italy: Family functioning, dyadic satisfaction, and child well-being. Sex Res Soc Policy J NSRC. 2015;12(3):202-12.

49. Bos H, Sandfort T. Children's Gender Identity in Lesbian and Heterosexual Two-Parent Families. Sex Roles. 2010;62(1/2):114-26.

50. Bos H, Gelderen L, Gartrell N. Lesbian and heterosexual two-parent families: Adolescent-parent relationship quality and adolescent well-being. J Child Fam Stud. 2015;24(4):1031-46.

51. Bos HMW, Van Balen F. Children in planned lesbian families: Stigmatisation, psychological adjustment and protective factors. Cult Health Sex. 2008;10(3):221-36.

52. Bos H, Gartrell N. Adolescents of the USA National Longitudinal Lesbian Family Study: Can Family Characteristics Counteract the Negative Effects of Stigmatization? Fam Process. 2010;49(4):559-72.

53. van Gelderen L, Gartrell NN, Bos HMW, Hermanns JMA. Stigmatization and Promotive Factors in Relation to Psychological Health and Life Satisfaction of Adolescents in Planned Lesbian Families. J Fam Issues. 2013;34(6):809-27.

54. Goldberg NG, Bos HMW, Gartrell NK. Substance use by adolescents of the USA National Longitudinal Lesbian Family Study. J Health Psychol. 2011;16(8):1231-40.

55. van Gelderen L, Gartrell N, Bos HMW, van Rooij FB, Hermanns JMA. Stigmatization associated with growing up in a lesbian-parented family: What do adolescents experience and how do they deal with it? Child Youth Serv Rev. 2012;34(5):999-1006. 
56. Bos HMW, Gartrell NK, van Balen F, Peyser H, Sandfort TGM. Children in planned lesbian families: A cross-cultural comparison between the United States and the Netherlands. Am J Orthopsychiatry. 2008;78(2):211-9.

57. Bos H, Van Balen F, Van Den Boom D, Sandfort T. Minority stress, experience of parenthood and child adjustment in lesbian families. J Reprod Infant Psychol. 2004;22(4):291-304.

58. Gartrell N, Rodas C, Deck A, Peyser H, Banks A. The National Lesbian Family Study: IV. Interviews with the 10-year-old children. Am J Orthopsychiatry. 2005;75(4):518-24.

59. Bos HMW, Gartrell NK, Peyser H, van Balen F. The USA National Longitudinal Lesbian Family Study (NLLFS): Homophobia, Psychological Adjustment, and Protective Factors. J Lesbian Stud. 2008;12(4):455-71.

60. Bos HMW, Gartrell NK. Adolescents of the US National Longitudinal Lesbian Family Study: the impact of having a known or an unknown donor on the stability of psychological adjustment. Hum Reprod. 2011;26(3):630-7.

61. Canneaux M, Missonnier S. Introduction. Spirale. 2017;84(4):11-8.

62. Guyotat J. Filiation et puerpéralité. Logiques du lien. Paris: PUF; 1995.

63. Zaouche-Gaudron C. Don de gamètes et aspect sociétal. 40 ans des CECOS, «De la naissance de l'AMP au futur »; 2013 Dec 12; Paris.

\section{Conflit d'intérêt : aucun.}


Tableau I. Caractéristiques des études et thèmes abordés.

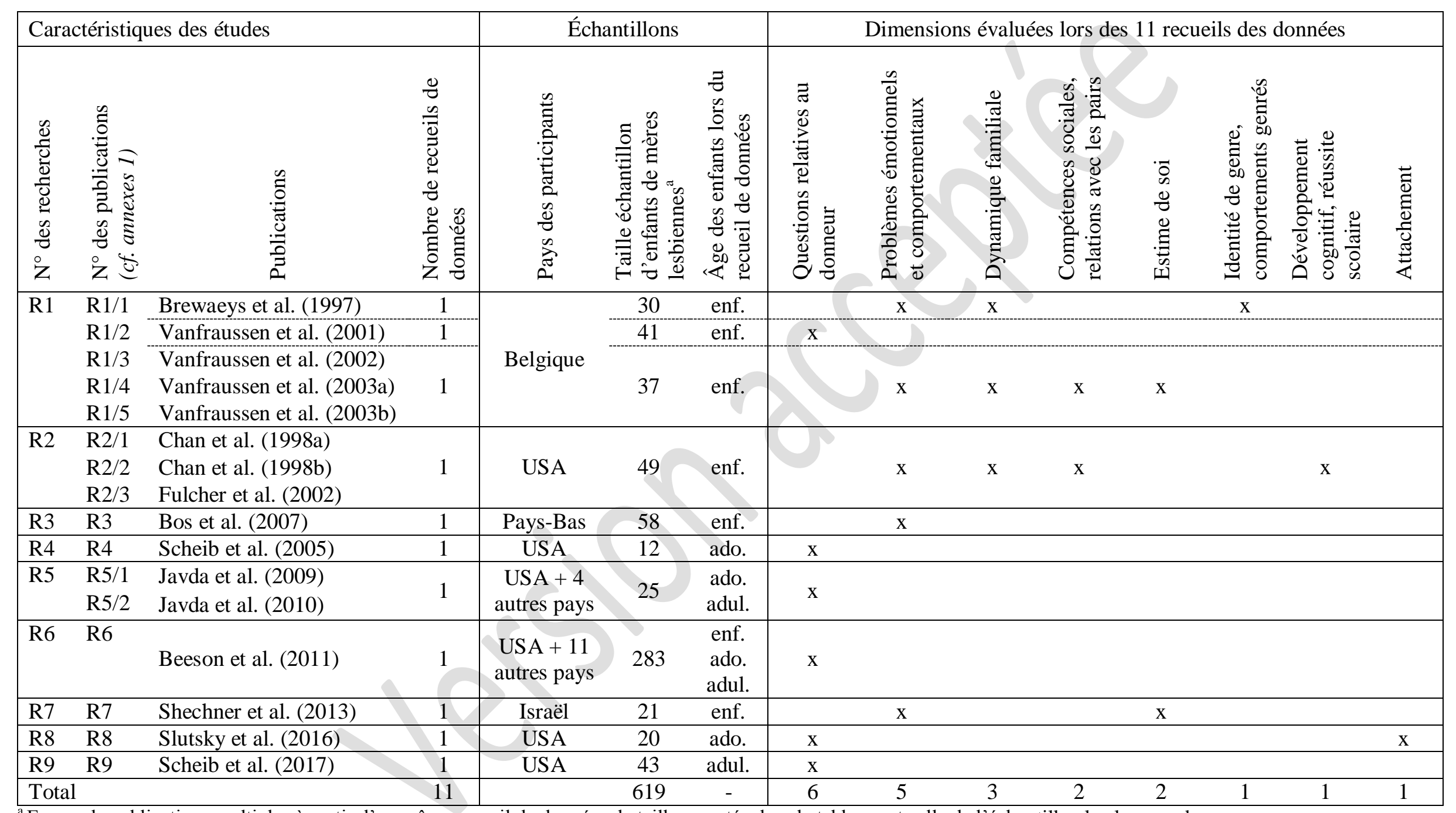

${ }^{\mathrm{a}}$ En cas de publications multiples à partir d'un même recueil de données, la taille reportée dans le tableau est celle de l'échantillon le plus grand. 


\section{Annexe 1. Principales caractéristiques des 16 publications retenues pour l'analyse et des 24 publications complémentaires}

\section{Notes pour les tableaux II et III.}

- seuls sont présentés les résultats concernant les enfants.

- les différences entre des groupes ou des scores ou les liens entre des scores mentionnés sont entendus comme « significatifs » d'un point de vue statistique.

- les astérisques $(*)$ indiquent que l'information nous a été communiquée par les auteurs ou est tirée d'une autre publication des auteurs.

Acronymes (certains créés par nous) et signes utilisés dans les tableaux II et III.

- dans les références et descriptifs des groupes : $\mathbf{F}=$ filles, $\mathbf{G}=$ garçons, $\mathbf{G P A}=$ Gestation Pour Autrui; $\mathbf{H}=$ hétérosexuels / hétérosexuelles, IND = information non disponible; L = lesbiens/lesbiennes; AMP = Assistance Médicale à la Procréation, T = Temps (phase de recueil de données).

- dans le descriptif des outils :

$\underline{\mathrm{ADP}}=$ Adolescent Disclosure to Parents; AEAA = Academics, Extracurricular Activities, and Aspirations; $\overline{\mathrm{A}} \mathrm{HS}=$ Anticipation of Homophobic Stigmatization; AQLS = Adolescent Quality of Life Scale; $\underline{\mathbf{B} S R I-R}=$ Bem Sex Role Inventory; $\underline{\mathbf{C}} \mathrm{BCL}=$ Child Behavior Checklist; $\mathrm{CCOF}=$ Contact with Children in Other Families with a lesbian or gay parent; CES-D = Center for Epidemiologic Studies Depression scale; CGOA = Contact with Grandparents and Other Adults; CPRS = Children's Peer Relation Scale; CRGL = Child-Rearing Goals List; CRPR = Child-Rearing Practices Report; DAS = Dyadic Adjustment Scale; DCIQ = Donor Conception Identity Questionnaire; DI = Disciplinary Issues; DIT = Disclosure Item; DOI = Donor Identity; DPCA $=$ Division of Professional and Childcare Activities; DWFT $=$ Division of Work and Family Tasks; $\underline{E} A T=$ Eating Attitudes Test $;$ EH $=$ Experiences with Homophobia; ERC = Emotion Regulation Checklist;

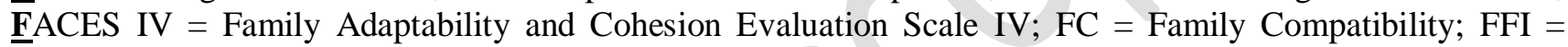
Friends and Family Interview; FI = Family Interactions; FR = Friendships; FRT = Family Relations Test;

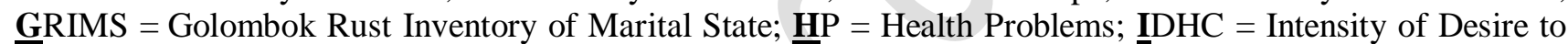
Have Child; IHP = Internalized Homophobia; IPPA = Inventory of Parent and Peer Attachment; LWMAT = Lock-Wallace Marital Adjustment Test; MGIQ = Multidimensional Gender Identity Questionnaire; MHP = Mental Health Problems; MPSAB = Mothers' Partnership Status After Breakup; MRM = Male Role Models; MSQ = Minority Stress Questionnaire; MSS = Marital Satisfaction Scale; NVOS = Nijmeegse vragenlijst voor de opvoedingssituatie (questionnaire de stress parental); OW = Overall Wellbeing; PACHIQ-P/Ch = ParentChild Interaction Questionnaire - Parent / Child; PASS = Parent Awareness Skills Survey; PCS = Parental Control Scale; $\mathrm{PCSC}=$ Perceived Competence Scale for Children; PFF = Plans For Families on their own; PGF = Peer Group Fit; PJ = Parental Justification; PSAI = Preschoolers' Activities Inventory; PSI/SF = Parenting Stress Index / Short Form; PQ = Partnership Questionnaire; PSPCSA = Pictorial Scale of Perceived Competence and Social Acceptance; $\underline{Q} P C R=$ Quality of the Parent-Child Relationship; QRSM = Quality of Relationship between Separated Mothers; $\underline{\mathbf{R} M}=$ Role Models; RSE = Rosenberg Self-Esteem scale; $\underline{\mathbf{S} A P}=$ Samen of Apart Questionnaire; SDQ = Strengths and Difficulties Questionnaire; SIAS = Social Interaction Anxiety Scale; SPRCASL = Supportive Presence, Respect for the Child's Autonomy, and Structure and Limitsetting; SQS = Sexual Questioning Scale; SS = Stigmatization Scale; STPI = State-Trait Personality Inventory; SUQ = Substance Use Questionnaire; $\underline{T} R F=$ Teacher's Report Form; $\underline{\text { WDW }}=$ Who Does What; WISC-R = Wechsler Intelligence Scale for Children-Revised; WPPSI-R = Wechsler Preschool and Primary Scale of

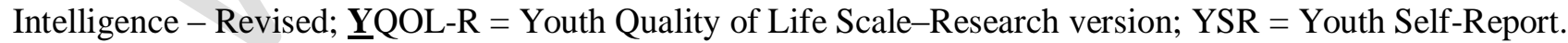

- évaluation portant au moins sur les enfants.

$\square$ évaluation portant uniquement sur les parents.

$\mathrm{QE} / \mathrm{E}=$ Questionnaire ou test rempli par les Enfants visant les Enfants.

$\mathrm{QP} / \mathrm{E}=$ Questionnaire rempli par les Parents visant les Enfants.

$\mathrm{QE} / \mathrm{E}-\mathrm{P}=$ Questionnaire ou test rempli par les Enfants visant les relations parent-enfant.

$\mathrm{QP} / \mathrm{E}-\mathrm{P}=$ Questionnaire rempli par les Parents visant les relations parents-enfants.

QObs/Enseigt $=$ Questionnaire rempli par des Observateurs ou des Enseignants/éducateurs visant les Enfants. $\mathrm{QP} / \mathrm{P}=$ Questionnaire ou test rempli par les Parents visant les Parents. 


\section{Tableau II. Principales caractéristiques des 16 publications retenues pour l'analyse}

\begin{tabular}{|c|c|c|c|}
\hline $\begin{array}{l}\mathbf{N}^{\circ} \text { de la } \\
\text { recherche / } \\
\mathrm{N}^{\circ} \text { de la } \\
\text { référence } \\
\text { - } \\
\text { Référence } \\
- \\
\text { Période de } \\
\text { recueil des } \\
\text { données }\end{array}$ & $\begin{array}{c}\text { Familles homoparentales } \\
\text { Nb enf et parents } \\
\text { Singleton vs fratrie } \\
\text { Genre } \\
\hat{\text { Age }} \\
\text { Rang de naissance } \\
\text { Identité donneur }\end{array}$ & $\begin{array}{c}\text { Groupe(s) de comparaison } \\
\text { Nb enf et parents } \\
\text { Méthode de conception } \\
\text { Singleton vs fratrie } \\
\text { Genre } \\
\text { Âge } \\
\text { Rang de naissance } \\
\text { Identité donneur }\end{array}$ & Outils \\
\hline $\begin{array}{c}\text { R1/1 } \\
(38) \\
\text { Brewaeys et } \\
\text { al. (1997) } \\
\text { T2 } \\
\text { Période de } \\
\text { recueil = IND }\end{array}$ & $\begin{array}{c}\bullet 30 \text { enf de } 30 \text { couples } \mathrm{L} \\
\text { Singletons ou issus de fratrie } \\
15 \mathrm{G} \text { et } 15 \mathrm{~F} \\
\text { Age moy. }=5 \text { ans } \\
1^{\text {ers }} \text { nés }=\text { oui } \\
\text { Donneur anonyme }\end{array}$ & $\begin{array}{c}-38 \text { enf de } 38 \text { couples } \mathrm{H} \\
\text { AMP } \\
\text { Singletons ou issus de fratrie } \\
26 \mathrm{G} \text { et } 12 \mathrm{~F} \\
\text { Age moy. }=5,4 \text { ans } \\
1^{\text {ers }} \text { nés }=\text { oui } \\
\text { Donneur anonyme } \\
\bullet 30 \text { enf de couples H } \\
\text { Conception naturelle } \\
\text { Singletons ou issus de fratrie } \\
11 \mathrm{G} \text { et } 19 \mathrm{~F} \\
\text { Age moy. }=5 \\
1^{\text {ers }} \text { nés }=\text { oui }\end{array}$ & $\begin{array}{l}\text { - } \mathrm{QE} / \mathrm{E}-\mathrm{P}=\mathrm{FRT} \\
\text { - } \mathrm{QP} / \mathrm{E}=\mathrm{CBCL}, \mathrm{PSAI} \\
\text { - } \mathrm{QP} / \mathrm{E}-\mathrm{P}=\mathrm{QPCR} \\
\square \mathrm{QP} / \mathrm{P}= \\
\mathrm{DI}, \mathrm{DPCA}, \text { GRIMS }\end{array}$ \\
\hline
\end{tabular}

- FRT : pas de différence de sentiments positifs et négatifs envers les parents entre enfants de mères L et ceux des deux groupes de comparaison.

- CBCL : pas de différence de problèmes comportementaux entre enfants de mères L et ceux des deux groupes de comparaison et ceux de la population de référence de l'outil.

- PSAI : pas de différence de comportements sexués entre les enfants de mères L et ceux des deux groupes de comparaison.

- QPCR : qualité des interactions mère non biologique-enfant dans les familles de mères L plus élevée que celle des interactions père-enfant dans les deux groupes de comparaison. Pas de différence de qualité entre interactions mère biologique-enfant dans les familles de mères lesbiennes et interactions mère-enfant dans les deux groupes de comparaison.

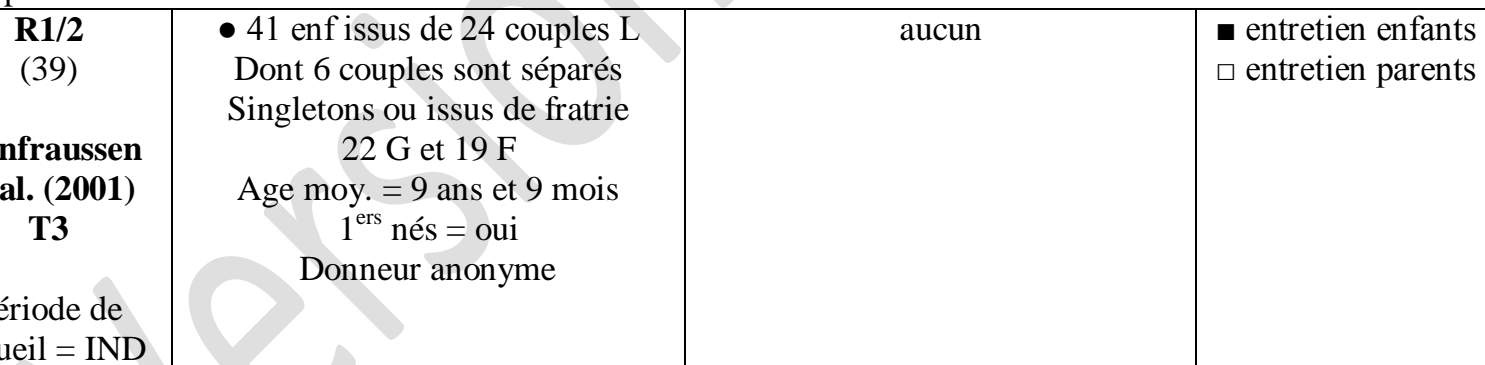

recueil $=$ IND

Tous les enfants sont informés qu'ils ont été conçus par AMP. 54\% d'entre eux préfèrent que le donneur soit anonyme, $27 \%$ souhaiteraient connaître son identité et $19 \%$ souhaiteraient des informations non identifiantes (physiques ou de personnalité). 63\% des enfants ne parlent jamais du donneur avec leurs mères. Les enfants souhaitent plus souvent que leurs mères obtenir des informations identifiantes ou non-identifiantes à propos du donneur.

\section{R1/3}

(40)

Vanfraussen et al. (2002)

T4

Période de recueil $=$ IND

- PCSC : pas de différence d'estime de soi globale, de sentiment d'acceptation sociale, de sentiment de compétences scolaires, physiques, sportives, de comportements et de relations avec les pairs) entre enfants de couples L et ceux du groupe de comparaison. 
- YSR (échantillon réduit à 14 enfants de mères L et 16 enfants de couples $\mathrm{H}$ ) : pas de différence de problèmes internalisés et externalisés, mais les enfants de mères L ont moins de comportements agressifs que ceux du groupe de comparaison. Aucun enfant de mères L n'obtient de scores dépassant le seuil pathologique déterminé pour l'outil.

- CBCL : pas de différence de problèmes internalisés, externalisés, de repli sur soi, de plainte somatique, d'anxiété/dépression, de problèmes sociaux, de problèmes de raisonnement, de problèmes d'attention ou de comportements délinquants. 7 enfants de mères L ont des scores de problèmes totaux qui dépassent le seuil pathologique, 8 au niveau des problèmes internalisés et 5 au niveau des problèmes externalisés (contre respectivement 7, 5 et 6 chez les enfants de couples $H$ ).

- TRF (échantillon réduit à 28 enfants de mères L et 31 enfants de couples $\mathrm{H}$ ) : les enseignants perçoivent davantage de problèmes totaux et de problèmes d'attention chez les enfants de mères L que chez ceux du groupe de comparaison.

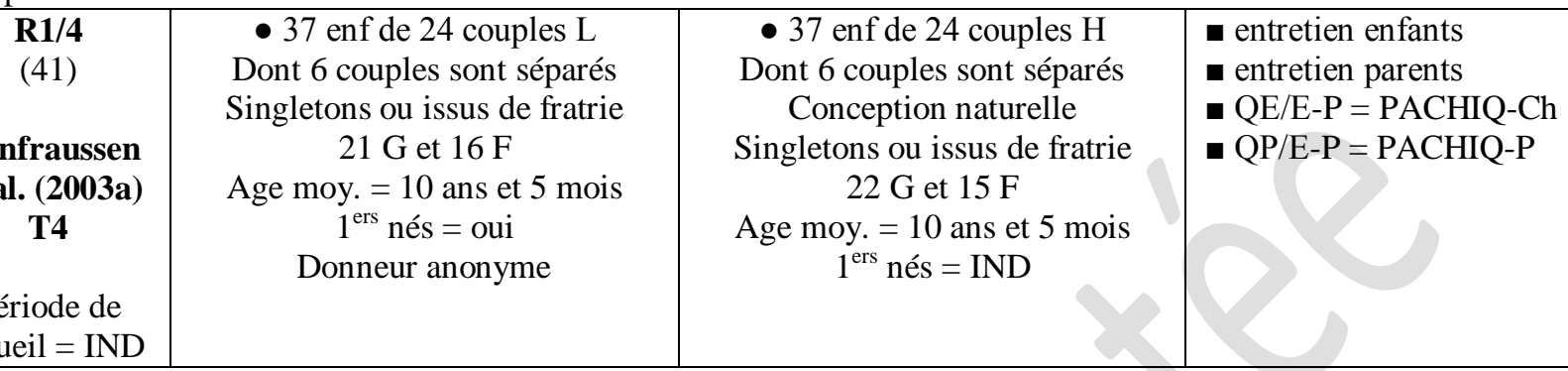

recueil $=$ IND

- PACHIQ-Ch : pas de différence de qualité des relations parent-enfant (comportements et sentiments) entre les familles de mères $\mathrm{L}$ et le groupe de comparaison.

- PACHIQ-P : pas de différence de qualité des relations parent-enfant (comportements et sentiments) entre les familles de mères L et le groupe de comparaison.

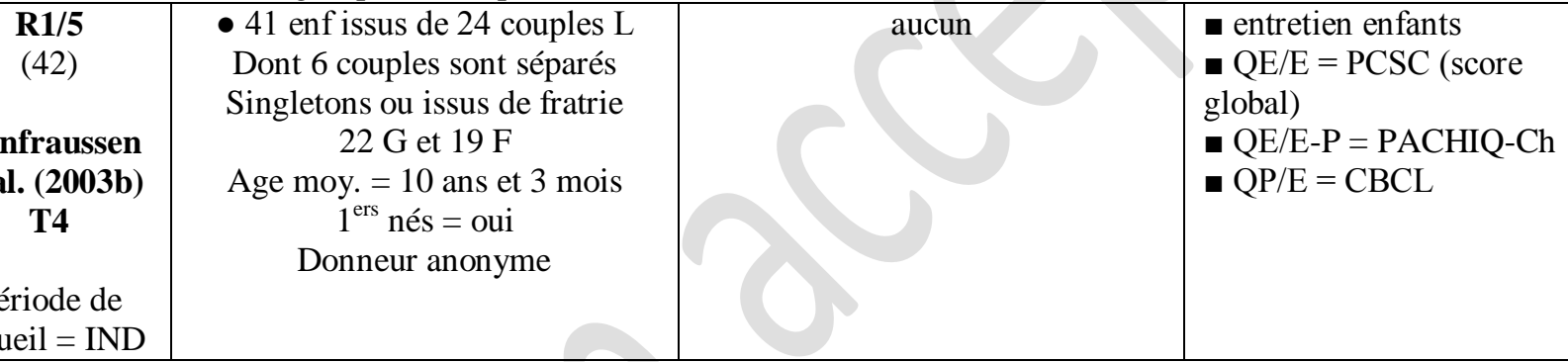

- PCSC : pas de différence d'estime de soi globale entre les enfants qui souhaitent avoir des informations sur le donneur et ceux qui ne le souhaitent pas.

- PACHIQ-Ch : pas de différence de qualité de relation parent-enfant entre les enfants qui souhaitent avoir des informations sur le donneur et ceux qui ne le souhaitent pas.

- CBCL : pas de différence de problèmes internalisés, externalisés et totaux entre les enfants qui souhaitent avoir des informations sur le donneur et ceux qui ne le souhaitent pas.

\begin{tabular}{|c|c|c|c|}
\hline $\begin{array}{c}\begin{array}{c}\mathbf{R} 2 / \mathbf{1} \\
(32)\end{array} \\
\text { Chan et al. } \\
(\mathbf{1 9 9 8 a}) \\
\text { Recueil entre } \\
1995 \text { et } 1996^{*}\end{array}$ & $\begin{array}{c}\bullet 30 \text { enf de } 30 \text { couples L } \\
\text { Singletons* } \\
20 \mathrm{G} \text { et } 10 \mathrm{~F} \\
\text { Age moy. }=7,1 \text { ans }(\mathrm{e}-\mathrm{t}=1,6) \\
1^{\text {ers }} \text { nés }=\text { oui } \\
\text { Donneur }=\text { IND } *\end{array}$ & $\begin{array}{c}\bullet 16 \text { enf de } 16 \text { couples } \mathrm{H} \\
\text { AMP } \\
\text { Singletons } * \\
10 \mathrm{G} \text { et } 6 \mathrm{~F} \\
\text { Age moy. }=7,9 \text { ans }(\mathrm{e}-\mathrm{t}=1,8) \\
1^{\text {ers }} \text { nés }=\text { oui } \\
\text { Donneur }=\text { IND } *\end{array}$ & $\begin{array}{l}\text { - } \mathrm{QP} / \mathrm{E}=\mathrm{CBCL} \\
\text { QObs/Enseigt }=\mathrm{TRF} \\
\square \mathrm{QP} / \mathrm{P}=\mathrm{LWMAT}, \\
\mathrm{WDW}\end{array}$ \\
\hline \multicolumn{4}{|c|}{$\begin{array}{l}\text { - CBCL et TRF : pas de différence entre les enfants de couples L et ceux du groupe de comparaison. En moyenne, } \\
\text { d'après les évaluations des parents et des enseignants, le fonctionnement des enfants est ajusté (les scores moyens de } \\
\text { problèmes internalisés, externalisés et totaux se situent en dessous du seuil de pathologie fixé pour les outils, les } \\
\text { scores moyens de compétences sociales et de performances scolaires sont au-dessous du seuil pathologique). } \\
\text { - TRF et WDW : pour les enfants de couples L et ceux du groupe de comparaison, plus les parents non-biologiques } \\
\text { sont satisfaits de la répartition des tâches ménagères, plus les scores de problèmes externalisés sont faibles. Pour les } \\
\text { enfants de couples L : plus les mères biologiques sont satisfaites de la répartition des tâches ménagères, moins elles } \\
\text { rapportent de problèmes externalisés; plus les mères non-biologiques sont satisfaites de la répartition des tâches } \\
\text { ménagères et des prises de décision liées à la famille, moins elles rapportent de problèmes externalisés; plus les } \\
\text { mères non-biologiques participent aux tâches liées à l'enfant, moins les mères biologiques rapportent de problèmes } \\
\text { externalisés. }\end{array}$} \\
\hline
\end{tabular}




\begin{tabular}{|c|c|c|c|}
\hline $\begin{array}{l}\qquad \begin{array}{l}\mathbf{R} 2 / \mathbf{2} \\
(33)\end{array} \\
\text { Chan et al. } \\
(\mathbf{1 9 9 8 b}) \\
\text { Recueil entre } \\
1995 \text { et } 1996^{*}\end{array}$ & $\begin{array}{c}\text { - } 34 \text { enf de } 34 \text { couples L } \\
\text { Singletons } * \\
1^{\text {ers }} \text { nés }=\text { oui } \\
\text { Donneur }=\text { IND* } \\
\text { - } 21 \text { enf de } 21 \text { mères L seules } \\
\text { Singletons* } \\
1^{\text {ers }} \text { nés }=\text { oui } \\
\text { Donneur }=\text { IND* } \\
\\
\text { Pour les deux groupes } \\
\text { confondus : } \\
37 \text { G et } 18 \mathrm{~F} \\
\text { Age moy. }=7,5 \text { ans }(e-t=1,8)\end{array}$ & $\begin{array}{c}\text { - } 16 \text { enf de } 16 \text { couples H } \\
\text { AMP } \\
\text { Singletons } \\
1^{\text {ers }} \text { nés }=\text { oui } \\
\text { Donneur }=\text { IND* } \\
\text {-9 enf de } 9 \text { mères H seules } \\
\text { AMP } \\
\text { Singletons } * \\
1^{\text {res }} \text { nés }=\text { oui } \\
\text { Donneur }=\text { IND* } \\
\\
\text { Pour les deux groupes } \\
\text { confondus : } \\
17 \text { G et } 8 \mathrm{~F} \\
\text { Age moy. }=7,8 \text { ans }(e-t=1,9)\end{array}$ & $\begin{array}{l}\text { - } \mathrm{QP} / \mathrm{E}=\mathrm{CBCL} \\
\text { QObs/Enseigt }=\mathrm{TRF} \\
\text { - } \mathrm{QP} / \mathrm{E}-\mathrm{P}=\mathrm{PSI} / \mathrm{SF} \\
\square \mathrm{QP} / \mathrm{P}=\mathrm{CES}-\mathrm{D}, \\
\text { LWMAT, PQ, RSE }\end{array}$ \\
\hline
\end{tabular}

- CBCL et TRF : pas de différence de problèmes de comportements internalisés, externalisés et totaux entre enfants de mères L (couples et mères seules confondues) et ceux de parents $\mathrm{H}$ (couples et mères seules confondus); au regard des populations normales de référence des outils, l'ensemble des enfants a un fonctionnement ajusté, les scores de compétences sociales sont plus élevés et ceux de problèmes comportementaux sont plus faibles que ceux de la population clinique de référence.

- CBCL / TRF et PSI/SF, CES-D, LWMAT, PQ, RSE : plus les interactions parent-enfant sont décrites comme dysfonctionnelles par les parents et plus le stress parental est élevé, plus les enfants ont de problèmes externalisés (les autres dimensions parentales n'apparaissent pas liées aux scores de problèmes des enfants).

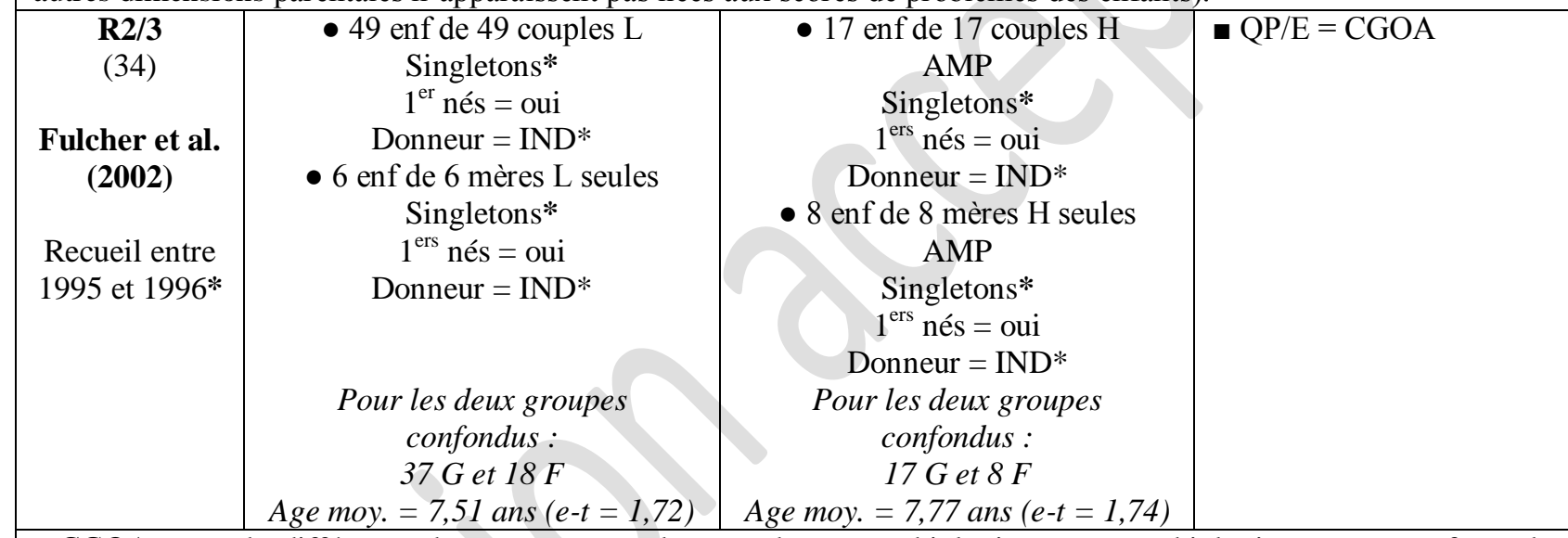

- CGOA : pas de différence de contacts avec les grands-parents biologiques et non-biologiques entre enfants de mères $\mathrm{L}$ (couples et mères seules confondus) et ceux de parents $\mathrm{H}$ (couples et mères seules confondus). Pas de différence de contacts avec d'autres adultes (amis des parents, voisins...) entre enfants de mères L (couples et mères seules confondus) et ceux de parents $\mathrm{H}$ (couples et mères seules confondus).

\begin{tabular}{|c|c|c|c|}
\hline $\begin{array}{c}\text { R3 } \\
(37) \\
\text { Bos \& } \\
\text { Hakvoort } \\
\text { (2007) } \\
\text { T1 } \\
\\
\text { Recueil en } \\
2001^{*}\end{array}$ & $\begin{array}{c}\bullet 52 \text { enf de } 52 \text { couples } \mathrm{L} \\
\text { Singletons ou issus de fratrie } \\
34 \mathrm{G} \text { et } 18 \mathrm{~F} \\
\text { Age moy. }=5,99 \text { ans }(\mathrm{e}-\mathrm{t}=1,33) \\
1^{\text {ers }} \text { nés }=\text { oui }^{*} \\
\text { Donneur anonyme* }\end{array}$ & $\begin{array}{l}-48 \text { enf de } 48 \text { couples } \mathrm{L} \\
\text { Auto-insémination } \\
\text { Singletons ou issus de fratrie } \\
18 \text { G et } 30 \mathrm{~F} \\
\text { Age moy. }=5,6 \text { ans }(\mathrm{e}-\mathrm{t}=1,40) \\
1^{\text {ers }} \text { nés }=\text { oui* } \\
\text { Donneur connu* }\end{array}$ & $\begin{array}{l}\text { - } \mathrm{QP} / \mathrm{E}=\mathrm{CBCL} \\
\square \mathrm{QP} / \mathrm{P}=\mathrm{CRPR}, \mathrm{MSQ}, \\
\mathrm{NVOS}, \mathrm{PJ}\end{array}$ \\
\hline \multicolumn{4}{|c|}{$\begin{array}{l}\text { - 52\% des enfants ont été conçus par insémination artificielle avec donneur (donneur anonyme) et } 48 \% \text { des enfants } \\
\text { ont été conçus par auto-insémination du sperme d'un donneur connu. } \\
\text { - CBCL : pas de différence de problèmes de comportements internalisés, externalisés et totaux entre enfants avec } \\
\text { donneur connu et ceux avec donneur anonyme (pour les filles comme pour les garçons). Les enfants avec donneur } \\
\text { connu ont des scores de problèmes sociaux plus élevés que ceux avec donneur anonyme (pour les garçons comme } \\
\text { pour les filles) (pas de différences sur les autres échelles). Chez les enfants avec donneur connu, les garçons ont des } \\
\text { scores de problèmes d'attention plus élevés que les filles (cette différence n'apparait pas chez les enfants avec } \\
\text { donneur anonyme). }\end{array}$} \\
\hline
\end{tabular}




\begin{tabular}{|c|c|c|c|}
\hline $\begin{array}{c}\text { R4 } \\
(35) \\
\text { Scheib, } \\
\text { Riordan, \& } \\
\text { Rubin (2005) } \\
\text { Recueil entre } \\
2000 \text { et } 2001^{*}\end{array}$ & $\begin{array}{c}\bullet 12 \text { enf de couples } \mathrm{L} \\
\text { Singletons ou issus de fratrie } \\
8 \mathrm{G} \text { et } 4 \mathrm{~F} \\
\text { Age moy. }=14,5(\mathrm{e}-\mathrm{t}=1,4) \\
1^{\text {ers }} \text { nés }=\text { une partie des enf* } \\
\text { Donneur = identité accessible à } \\
18 \text { ans }\end{array}$ & $\begin{array}{c}\bullet 6 \text { enf de couples H } \\
\text { AMP } \\
\text { Singletons ou issus de fratrie } \\
4 \text { G et } 2 \mathrm{~F} \\
\text { Age moy. }=14,6(\mathrm{e}-\mathrm{t}=1,2) \\
1^{\text {ers }} \text { nés }=\text { une partie des enf* } \\
\text { Donneur connu } \\
\text { - } 11 \text { enf de mères seules } \\
\text { (orientation sexuelle = IND*) } \\
\text { AMP } \\
\text { Singletons ou issus de fratrie } \\
\quad 6 \text { G et } 5 \mathrm{~F} \\
\text { Age moy. }=15,5(\mathrm{e}-\mathrm{t}=1,5) \\
1^{\text {ers }} \text { nés }=\text { une partie des enf* } \\
\text { Donneur connu }\end{array}$ & - entretien enfants \\
\hline
\end{tabular}

- connaissances de leur conception : 75,9\% ont le sentiment d'avoir toujours su qu'ils ont été conçus par AMP, les autres se souviennent l'avoir appris entre 4 et 9,5 ans, sans différence entre les 3 groupes. Pour chacun des 3 groupes, apprendre et savoir l'origine de leur naissance n'a pas affecté, ou alors positivement, leur relation avec leur mère biologique, et n'a pas affecté leur relation avec leur père ou leur mère non-biologique.

- affects liés à la connaissance de leur conception : la plupart des enfants se sentent à l'aise avec le fait d'avoir été conçu par AMP, quelques-uns sont neutres, surtout parce qu'ils estiment que cela n'affecte pas leur vie (44\%), mais aussi parce qu'ils se sentent très aimés dans leur famille et uniques $(40 \%)$. Certains se sentent différents mais ne ressentent pas d'affect particulier à cet égard $(8 \%)$ et d'autres se sentent différents et un peu moins à l'aise à cette idée $(8 \%)$. Pas de différence entre les 3 groupes quant à ces résultats.

- divulgation de leur conception : les enfants indiquent qu'ils ont modérément ou peu parlé de leur conception autour d'eux, et lorsqu'ils l'ont fait c'est le plus souvent avec des membres de la famille étendue, un peu moins avec leurs amis et peu avec leurs enseignants. Les réactions de leur entourage sont évaluées comme plutôt positives, davantage par les enfants de couples $\mathrm{L}$ et de mères seules que par ceux de couples $\mathrm{H}$.

- termes pour nommer le donneur : pour parler de leur donneur, $24,1 \%$ des enfants utilisent le terme de « donneur », $24,1 \%$ de «père de naissance / biologique », 24,1\% de «père / papa »; 13,8\% des enfants utilisent plusieurs de ces termes. Pas de différence entre les 3 groupes quant à ces résultats.

- sentiments à propos du donneur : en moyenne, les sentiments envers le donneur sont plutôt positifs (de neutres à très positifs). Les enfants de mères seules ont des sentiments plus positifs que ceux de couples L. 86,2\% se disent curieux à propos du donneur. 51,7\% lui sont reconnaissants, et les enfants de mères seules et de couples $\mathrm{H}$ sont plus reconnaissants que ceux de mères L. $34,5 \%$ des enfants se disent anxieux et préoccupés à l'idée de rencontrer le donneur, $31 \%$ se disent excités à l'idée de le rencontrer et d'en apprendre davantage sur lui. 10,3\% des enfants estiment que le donneur est une personne importante dans leur vie, et 21,4\% disent n'avoir jamais vraiment pensé au donneur, $6,9 \%$ disent ne rien ressentir à son égard. $17,2 \%$ des enfants disent ne pas se soucier du donneur. Un enfant $(3,4 \%)$ ressent de la colère et un enfant $(3,4 \%)$ du ressentiment envers lui.

- description du meilleur / pire donneur possible : le meilleur donneur que les enfants imaginent est décrit par 76\% d'entre eux avec des traits physiques ou de personnalité positifs (ex : intelligent, heureux) et par 44\% par des interactions positives avec lui (ex : envie de rencontrer l'enfant, partage d'intérêts), et aucun enfant n'a décrit le donneur idéal comme un homme qui souhaiterait jouer le rôle de père; le pire donneur que les enfants imaginent est décrit par $80 \%$ d'entre eux avec des traits physiques ou de personnalité négatifs (ex : violent, malheureux), et par $40 \%$ d'entre eux par des interactions négatives avec lui (ex : sans intérêt pour l'enfant, intrusif);

- questionnements à propos du donneur : $82,87 \%$ des enfants s'interrogent sur la personnalité du donneur, 41,4\% sur son apparence physique, $34,5 \%$ sur sa famille, $24,1 \%$ sur leur ressemblance avec lui, $17,2 \%$ sur son envie de les rencontrer, $10,3 \%$ sur sa santé, $10,7 \%$ sur ses motivations à être donneur de sperme, $6,9 \%$ son nom, et 1 enfant $(3,4 \%)$ s'il a déjà pensé à lui. Seuls des enfants de mères seules souhaitent connaître les motivations du donneur à l'être.

- informations souhaitées à propos du donneur : au moment de la délivrance des informations relatives au donneur, en complément de son identité, les enfants souhaiteraient surtout avoir sa photo $(96,6 \%)$, sa situation familiale actuelle $(89,7 \%)$, ses sentiments à l'idée d'être contacté par l'enfant $(79,3 \%)$ (moins chez les enfants de couples L que chez les autres), sa santé $(69 \%)$, son histoire familiale $(65,5 \%)$

- intérêt pour les autres enfants issus du même donneur : 89,7\% des enfants se disent de « modérément » à «très intéressés » d'avoir des contacts avec d'éventuels autres enfants issus du donneur et $85,7 \%$ de les rencontrer. Plus les enfants sont intéressés de rencontrer le donneur, plus ils sont intéressés de rencontrer d'éventuels autres enfants issus de lui.

- projet de demander l'identité du donneur : 86,2\% des enfants se disent de «modérément » à « très » intéressés de demander l'identité du donneur. Les enfants de mères seules se disent plus intéressés que ceux de couples L. Les enfants supposent que leurs parents réagiraient de façon plutôt positive à la leur demande d'information. 
- modalités de contact avec le donneur : 41,4\% des enfants projettent de contacter le donneur à leurs 18 ans, 20,7\% à 18 ans ou plus tard, et $27,6 \%$ ne sont pas sûrs. $60,7 \%$ des enfants espèrent qu'ils pourront contacter le donneur avec l'aide de la banque de sperme, $28,6 \%$ par courrier électronique ou postal, 7,1\% en personne et 3,6\% par téléphone.

- motivations pour contacter le donneur : 82,8\% des enfants souhaitent contacter le donneur pour obtenir plus d'informations sur lui, 82,6\% pour obtenir des informations sur sa vie, 26,1\% pour savoir s'ils lui ressemblent, $66,7 \%$ pour apprendre davantage sur eux-mêmes, $62,1 \%$ pour en apprendre davantage sur, et peut-être rencontrer, sa famille, 51,4\% pour être en relation avec lui. Les enfants de mères seules souhaitent davantage rencontrer le donneur pour apprendre davantage sur eux-mêmes que les enfants de couples $\mathrm{H}$ et $\mathrm{L}$.

\begin{tabular}{|c|c|c|c|}
\hline $\begin{array}{c}\text { RJ/1 } \\
(46) \\
\text { Jadva, } \\
\text { Freeman, } \\
\text { Kramer, \& } \\
\text { Golombok } \\
\text { (2009) }\end{array}$ & $\begin{array}{c}\bullet 25 \text { enf de couples L } \\
\text { Singletons ou issus de fratrie* } \\
1^{\text {ers }} \text { nés }=\text { IND* } \\
\text { Donneur = IND }\end{array}$ & $\begin{array}{c}\bullet 96 \text { enf de couples H } \\
\text { AMP } \\
\text { Singletons ou issus de fratrie* } \\
1^{\text {ers }} \text { nés }=\text { IND* } \\
\text { Donneur = IND } \\
\bullet 38 \text { enf de mères seules } \\
\text { AMP } \\
\text { Singletons ou issus de fratrie* } \\
1^{\text {ers }} \text { nés }=\text { IND* } \\
\text { Donneur }=\text { IND }\end{array}$ & \multirow[t]{2}{*}{$\begin{array}{l}\text { - expérience en tant } \\
\text { qu'enfant conçu par AMP } \\
\text { et recherche du donneur } \\
\text { ou de ses autres } \\
\text { progénitures }\end{array}$} \\
\hline $\begin{array}{l}\text { Recueil en } \\
\text { 2007* }\end{array}$ & \multicolumn{2}{|c|}{$\begin{array}{l}\text { Pour les } 3 \text { groupes confondus : } \\
\qquad 42 \mathrm{G} \text { et } 123 \mathrm{~F} \\
\quad \text { Age }=\text { de } 13 \text { à } 61 \text { ans } \\
\text { Age moy. }=22 \text { ans }(\mathrm{e}-\mathrm{t}=10)\end{array}$} & \\
\hline \multicolumn{4}{|c|}{$\begin{array}{l}\text { - } 59 \% \text { des enfants de couples } \mathrm{H} \text { ont appris leur mode de conception avant l'âge de } 15 \text { ans (et } 67 \% \text { avant } 18 \text { ans), } \\
\text { contre } 100 \% \text { dans les deux autres groupes. } \\
\text { - } 56 \% \text { des enfants de couples L disent avoir toujours connu leur mode de conception, contre } 45 \% \text { de ceux de mères } \\
\text { seules et } 40 \% \text { de ceux de couples } \mathrm{H} \text {. } \\
\text { - pas de différence entre les enfants des } 3 \text { groupes dans l'utilisation des termes pour parler du donneur (donneur = } \\
36 \% \text {, père }=8 \% \text {, père biologique }=5 \% \text {, papa }=2 \% \text {, père donneur }=1 \% \text {, aucun terme }=47 \% \text { ). }\end{array}$} \\
\hline $\begin{array}{l}\text { Jadva, } \\
\text { Freeman, } \\
\text { Kramer, \& } \\
\text { Golombok } \\
\quad(2010)\end{array}$ & $\begin{array}{c}\bullet 25 \text { enf de couples L } \\
\text { Singletons ou issus de fratrie* } \\
\text { 1ers nés = IND* } \\
\text { Donneur = IND }\end{array}$ & $\begin{array}{c}\bullet 96 \text { enf de couples H } \\
\text { AMP } \\
\text { Singletons ou issus de fratrie* } \\
\text { lers nés = IND* } \\
\text { Donneur = IND } \\
\bullet 38 \text { enf de mères seules } \\
\text { AMP } \\
\text { Singletons ou issus de fratrie* } \\
\text { 1ers nés = IND* } \\
\text { Donneur = IND }\end{array}$ & \multirow[t]{2}{*}{$\begin{array}{l}\text { - expérience en tant } \\
\text { qu'enfant conçu par AMP } \\
\text { et recherche du donneur } \\
\text { ou de ses autres } \\
\text { progénitures }\end{array}$} \\
\hline $\begin{array}{l}\text { Recueil en } \\
2007 *\end{array}$ & Pour les 3 gr & $\begin{array}{l}\text { confondus: } \\
23 \mathrm{~F} \\
\text { à } 61 \text { ans } \\
\mathrm{ns}(\mathrm{e}-\mathrm{t}=10)\end{array}$ & \\
\hline \multicolumn{4}{|c|}{$\begin{array}{l}\text { - tous groupes confondus, } 78 \% \text { des enfants ont informé leur mère et } 60 \% \text { ont informé leurs amis qu'ils recherchaient } \\
\text { les autres progénitures de leur donneur. } \\
\text { - tous groupes confondus, } 79 \% \text { des enfants ont informé leur mère et } 58 \% \text { ont informé leurs amis qu'ils recherchaient } \\
\text { leur donneur. } \\
\text { - } 57 \% \text { des enfants de couples } \mathrm{H} \text { ont recherché les autres progénitures de leur donneur, contre } 25 \% \text { de ceux de mères } \\
\text { seules et } 14 \% \text { de ceux de couples L. } \\
\text { - } 63 \% \text { des enfants de couples } \mathrm{H} \text { ont recherché leur donneur, contre } 20 \% \text { de ceux de mères seules et } 13 \% \text { de ceux de } \\
\text { couples L. } \\
\text { - } 89 \% \text { des enfants de couples L ont informé leur mère non-biologique et } 22 \% \text { des enfants de couples H ont informé } \\
\text { leur père non-biologique qu'ils recherchaient les autres progénitures de leur donneur. } \\
\text { - } 65 \% \text { des enfants de couples L ont informé leur mère non-biologique et } 29 \% \text { des enfants de couples H ont informé } \\
\text { leur père non-biologique qu'ils recherchaient les autres progénitures de leur donneur. } \\
\text { - pas de différence de réaction quant à la recherche des autres progénitures du donneur ou du donneur lui-même entre } \\
\text { les mères des } 3 \text { groupes ni entre les mères non-biologiques des couples L et les pères des couples H. }\end{array}$} \\
\hline
\end{tabular}




\begin{tabular}{|c|c|c|c|}
\hline \multirow{2}{*}{ 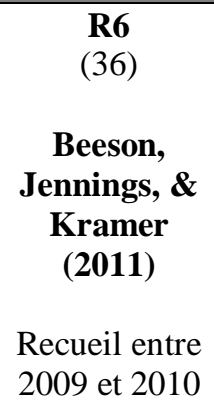 } & 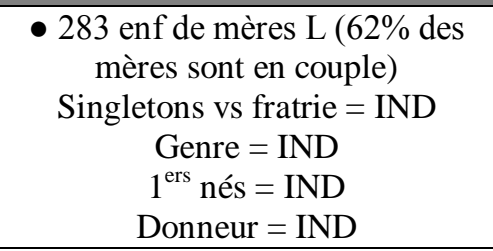 & $\begin{array}{l}-458 \text { enf de parents } \mathrm{H} \\
\text { AMP } \\
\text { Singletons vs fratrie = IND } \\
\text { Genre = IND } \\
1_{\text {ers }}^{\text {nés }=\text { IND }} \\
\text { Donneur = IND }\end{array}$ & \multirow[t]{2}{*}{$\begin{array}{l}\text { - expérience en tant } \\
\text { qu'enfant conçu par AMP } \\
\text { et recherche du donneur }\end{array}$} \\
\hline & \multicolumn{2}{|c|}{$\begin{array}{l}\text { Tous groupes confondus : } \\
\text { Age : de } 9 \text { à plus de } 40 \text { ans (53\% ont moins de } 18 \text { ans; } 60 \% \text { des } \\
\text { enfants de mères } L \text { et } 26 \% \text { de ceux de couples } \mathrm{H} \text { ont moins de } 18 \text { ans) }\end{array}$} & \\
\hline \multicolumn{4}{|c|}{ 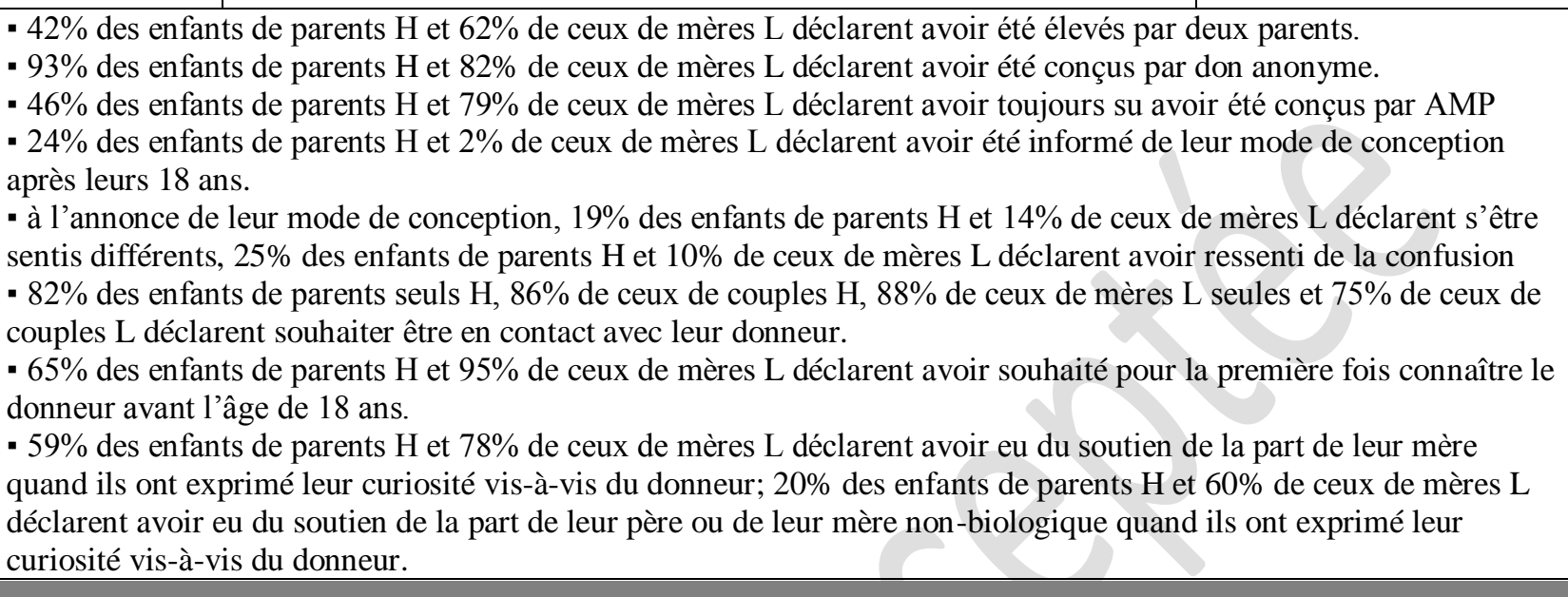 } \\
\hline $\begin{array}{c}\text { R7 } \\
\quad(43) \\
\text { Shechner, } \\
\text { Slone, Lobel, } \\
\text { \& Shechter } \\
\text { (2013) } \\
\text { Recueil entre } \\
2005 \text { et } 2008^{*}\end{array}$ & $\begin{array}{c}\bullet 21 \text { enf de } 21 \text { couples } \mathrm{L} \\
\text { Singletons vs fratrie = IND* } \\
11 \mathrm{G} \text { et } 10 \mathrm{~F} \\
\text { Age moy. }=6,07(\mathrm{e}-\mathrm{t}=1,64) \\
1^{\mathrm{ers}} \text { nés }=\text { IND* } \\
\text { Donneur anonyme } \\
\text { - } 15 \text { enf de } 15 \text { mères L seules } \\
\text { Singletons vs fratrie }=\mathrm{IND} * \\
8 \mathrm{G} \text { et } 7 \mathrm{~F} \\
\text { Age moy. }=6,70(\mathrm{e}-\mathrm{t}=1,13) \\
1^{\text {ers }} \text { nés }=\mathrm{IND} * \\
\text { Donneur anonyme }\end{array}$ & $\begin{array}{c}\text { - } 24 \text { enf de } 24 \text { couples } \mathrm{H} \\
\text { Conception naturelle } \\
\text { Singletons vs fratrie = IND* } \\
12 \mathrm{G} \text { et } 12 \mathrm{~F} \\
\text { Age moy. }=6,17(\mathrm{e}-\mathrm{t}=1,24) \\
1^{\text {ers }} \text { nés }=\mathrm{IND}^{*} \\
\text { - } 16 \text { enf de } 16 \text { mères H seules } \\
\text { AMP } \\
\text { Singletons vs fratrie }=\text { IND* } \\
8 \mathrm{G} \text { et } 8 \mathrm{~F} \\
\text { Age moy. }=6,84(\mathrm{e}-\mathrm{t}=1,37) \\
1^{\text {ers }} \text { nés }=\text { IND* } \\
\text { Donneur anonyme }\end{array}$ & $\begin{array}{l}\text { - } \mathrm{QE} / \mathrm{E}=\mathrm{CPRS} \\
\text { - } \mathrm{QE} / \mathrm{E}=\mathrm{PSPCSA} \\
\text { - } \mathrm{QP} / \mathrm{E}=\mathrm{CBCL}\end{array}$ \\
\hline \multicolumn{4}{|c|}{$\begin{array}{l}\text { - CPRS : moins de comportements antisociaux et de sentiment de solitude chez les enfants de mères L que chez ceux } \\
\text { de couples/mères H. } \\
\text { - PSPSCA : pas de différence de sentiment de compétences cognitive, physiques, dans la relation à la mère et dans } \\
\text { les relations avec les pairs entre enfants de mères L et ceux de couples H et mères H seules. } \\
\text { - CBCL : pas de différence de problèmes comportementaux internalisés et externalisés entre enfants de mères L et } \\
\text { ceux de couples/mères H, et les } 4 \text { groupes d'enfants se situent en dessous du seuil de pathologie de l'outil. }\end{array}$} \\
\hline $\begin{array}{l}\text { Slutsky, } \\
\text { Jadva, } \\
\text { Freeman, } \\
\text { Persaud, } \\
\text { Steele, Steele, } \\
\text {. . Golombok } \\
\quad \text { (2016) } \\
\text { Recueil entre }\end{array}$ & $\begin{array}{c}-7 \text { enf de couples } \mathrm{L} \text { et } 12 \text { enf de } \\
\text { mères L seules } \\
\text { Singletons ou issus de fratrie* } \\
4 \mathrm{G} \text { et } 15 \mathrm{~F} \\
\text { Age moy. }=14,18 \text { ans }(\mathrm{e}-\mathrm{t}=2,20) \\
1^{\text {ers }} \text { nés }=\text { IND* } \\
\text { Donneur anonyme }\end{array}$ & aucun & $\begin{array}{l}\text { - entretiens enfants } \\
\text { - QE/E = DCIQ, FFI }\end{array}$ \\
\hline
\end{tabular}


- $10,5 \%$ des enfants déclarent avoir toujours connu leur mode de conception, $47,4 \%$ ne se rappellent pas quand ils l'ont appris et $42,1 \%$ l'ont appris au plus tard à 7 ans.

- aucun enfant n'a de contact avec son donneur mais tous ont localisé au moins un autre enfant conçu à partir d'un don du même donneur.

- DCIQ : 37\% des enfants présentent un pattern d'attachement insécure

- DCIQ et FFI : plus le score d'attachement sécure est élevé, plus le score de curiosité envers le donneur l'est aussi; plus le score d'attachement insécure désorganisé est élevé, plus le score d'évitement des questions liées au mode de conception l'est aussi.

\begin{tabular}{|c|c|c|c|}
\hline $\begin{array}{c}\text { Scheib, Ruby, } \\
\text { \& Benward } \\
(2017)\end{array}$ & $\begin{array}{c}\bullet 117 \text { enf de couples L } \\
\text { Singletons ou fratrie } \\
\text { Genre }=\text { IND* } \\
18 \text { à } 29 \text { ans } \\
1^{\text {ers }} \text { nés }=\text { IND* } \\
\text { Donneur }=\text { identité accessible à } \\
18 \text { ans }\end{array}$ & $\begin{array}{c}\text { - } 73 \text { enf de couples H } \\
\text { AMP } \\
\text { Singletons ou fratrie } \\
\text { Genre = IND* } \\
18 \text { à } 29 \text { ans } \\
1^{\text {ers }} \text { nés }=\text { IND* } \\
\text { - } 43 \text { enf de mères seules } \\
\text { AMP } \\
\text { Singletons ou fratrie } \\
\text { Genre }=\text { IND* } \\
18 \text { à } 29 \text { ans } \\
1^{\text {ers }} \text { nés }=\text { IND* }\end{array}$ & - entretiens enfants \\
\hline \multicolumn{4}{|c|}{$\begin{array}{l}\text { - sur } 233 \text { enfants, } 85 \text { ( } 43 \text { enfants de couples L ( } 22 \mathrm{G} \text { et } 21 \mathrm{~F}), 17 \text { de couples } \mathrm{H} \text { (5G et } 12 \mathrm{~F}) \text { et } 25 \text { de mères seules }(6 \mathrm{G} \\
\text { et } 19 \mathrm{~F}))^{*} \text { ont entamé des démarches pour obtenir des informations sur leur donneur }(36 \%), 95 \% \text { d'entre eux sont les } \\
\text { premiers enfants nés de leurs parents } \\
\text { - les enfants de couples L }(36,5 \%) \text { et ceux de mères seules }(58,1 \%) \text { ont davantage entamé des démarches pour obtenir } \\
\text { des informations sur leur donneur que ceux de couples } \mathrm{H}(23,3 \%) \text {. } \\
\text { - } 49 \% \text { des enfants de mères L ayant entamé des démarches pour obtenir des informations sur leur donneur sont des } \\
\text { filles, contre } 76 \% \text { chez ceux de mères seules et } 71 \% \text { chez ceux de couples H* } \\
\text { - pas de différence de recherche d'identité du donneur selon que les enfants ont ou non au moins un parent du même } \\
\text { genre qu'eux. } \\
\text { - en se fondant sur des études antérieures, les auteurs estiment que } 65 \% \text { des couples H du programme ont dévoilé à } \\
\text { leur enfant qu'il a été conçu par AMP. Ils estiment ainsi que } 40 \% \text { des enfants qui étaient informés de leur méthode de } \\
\text { conception ont entamé les démarches pour obtenir des informations sur leur donneur. }\end{array}$} \\
\hline
\end{tabular}




\section{Tableau III. Principales caractéristiques des 24 publications complémentaires}

\begin{tabular}{|c|c|c|c|}
\hline $\begin{array}{c}\mathbf{N}^{\circ} \text { de la } \\
\text { recherche / } \\
\mathrm{N}^{\circ} \text { de la } \\
\text { référence } \\
\text { - } \\
\text { Référence } \\
\text { - } \\
\text { Période de } \\
\text { recueil des } \\
\text { données }\end{array}$ & $\begin{array}{c}\text { Familles homoparentales } \\
\text { Nb enf et parents } \\
\text { Singleton vs fratrie } \\
\text { Genre } \\
\text { Âge } \\
\text { Rang de naissance } \\
\text { Identité donneur }\end{array}$ & $\begin{array}{c}\text { Groupe(s) de comparaison } \\
\text { Nb enf et parents } \\
\text { Méthode de conception } \\
\text { Singleton vs fratrie } \\
\text { Genre } \\
\hat{A} g e \\
\text { Rang de naissance } \\
\text { Identité donneur }\end{array}$ & $\begin{array}{l}\quad \text { Outils } \\
\text { - évaluation portant au } \\
\text { moins sur les enfants } \\
\text { évaluation portant } \\
\text { uniquement sur les } \\
\text { parents }\end{array}$ \\
\hline $\begin{array}{c}\text { RC1/1 } \\
(57) \\
\text { Bos, Van Balen, } \\
\text { Van Den Boom, } \\
\text { \& Sandfort } \\
(\mathbf{2 0 0 4 )} \\
\text { T1 }\end{array}$ & $\begin{array}{c}\bullet 100 \text { enf de } 100 \text { couples } \mathrm{L} \\
\text { Singletons ou issus de fratrie } \\
52 \mathrm{G} \text { et } 48 \mathrm{~F} \\
\text { Age moy. }=5,8 \text { ans }(\mathrm{e}-\mathrm{t}=1,37) \\
1^{\text {ers }} \text { nés }=\text { oui } * \\
\text { Donneur connu }(42 \%) \text { et } \\
\text { donneur anonyme }(52 \%)^{*}\end{array}$ & aucun & $\begin{array}{l}-\mathrm{QP} / \mathrm{E}=\mathrm{CBCL} \\
\square \mathrm{QP} / \mathrm{P}=\mathrm{IHP}, \mathrm{MSQ}, \\
\mathrm{NVOS}, \mathrm{PJ}, \mathrm{SAP}\end{array}$ \\
\hline
\end{tabular}

- CBCL : dans l'ensemble, du point de vue des problèmes de comportements internalisés, externalisés et totaux, les enfants de l'échantillon ont un fonctionnement ajusté. Pas de différence d'évaluation entre mères biologiques et mères non-biologiques.

- CBCL et IHP / MSQ / NVOS / PJ / SAP : plus les mères ont fait l'expérience du rejet en tant que mères lesbiennes au sein de l'environnement social, plus les niveaux de problèmes de comportements internalisés, externalisés et totaux chez les enfants sont élevés. Aucune autre dimension parentale (stigmatisation sociale perçue, homophobie internalisée, sentiment de charge parentale, sentiment de compétence parentale, sentiment de pression sociale à démontrer ses qualités de parents) n'est corrélée aux scores de problèmes de comportements.

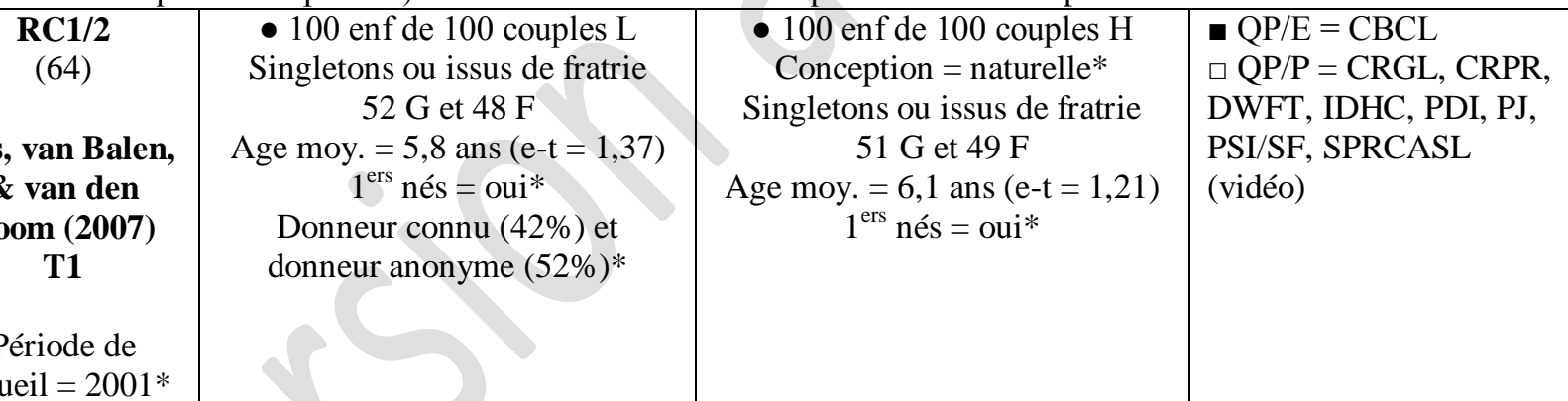

- CBCL : pas de différence de problèmes de comportements internalisés, externalisés et totaux entre enfants de couples L et ceux de couples H. Pour les deux groupes confondus (enfants de couples L et ceux de couples H), plus les parents sont satisfaits de l'implication parentale de leur partenaire et soutenus par lui/elle, moins les enfants ont de problèmes internalisés; plus les parents sont satisfaits de l'implication parentale de leur partenaire, moins les enfants ont de problèmes externalisés et totaux.

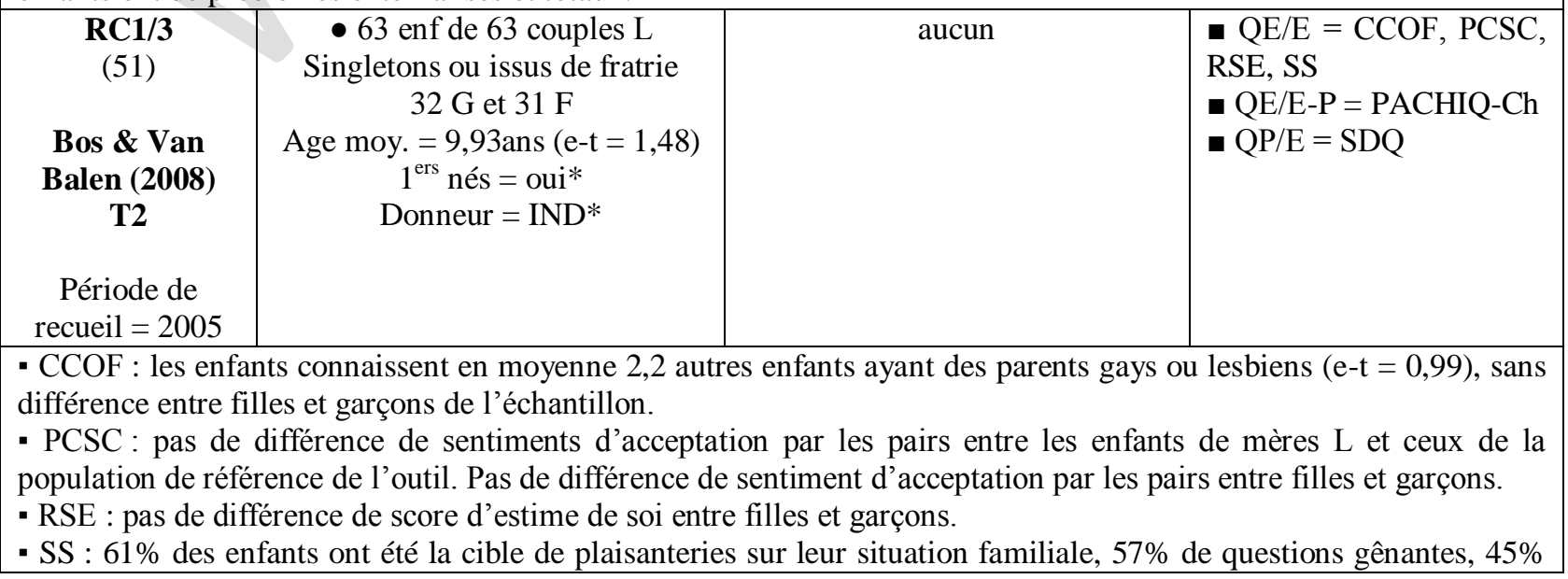


d'expressions blessantes pour parler de l'orientation sexuelle de leurs mères, $31 \%$ de médisance, $26 \%$ d'exclusion, $21 \%$ de remarques désapprobatrices (les autres formes de stigmatisation sont moins fréquentes). Les filles ont davantage que les garçons le sentiment de faire l'objet de médisance (pas de différence concernant les autres formes de stigmatisation).

- PACHIQ-Ch: pas de différence de qualité des relations enfants-mères biologiques et enfants-mères non biologiques, ni pour les filles ni pour les garçons. Pour l'ensemble de l'échantillon, la qualité des relations ne diffère pas de celle de la population de référence de l'outil.

- SDQ : les garçons ont des scores de conduites problématiques et d'hyperactivité plus élevés que les filles. 4 enfants ont des scores de conduites problématiques qui dépassent le seuil pathologique, 10 enfants au niveau des problèmes émotionnels et 4 enfants au niveau de l'hyperactivité.

- SDQ et CCOF / PCSC / SS / PACHIQ-Ch : pour les garçons, plus le niveau de stigmatisation est élevé, plus celui d'hyperactivité est élevé; plus les enfants se sentent acceptés par leurs pairs, plus leur estime de soi est élevée. Pour les filles, l'estime de soi est plus élevée lorsque le niveau de stigmatisation est faible, la qualité des relations avec les parents est élevée, le sentiment d'acceptation par les pairs est élevé; plus les filles ont de contact avec d'autres enfants de familles homoparentales, moins leur niveau d'hyperactivité est élevé; plus le sentiment d'acceptation par les pairs est élevé, moins le niveau de conduites problématiques est élevé.

\begin{tabular}{|c|c|c|c|}
\hline $\begin{array}{c}\text { Bos, Gartrell, } \\
\text { van Balen, } \\
\text { Peyser, \& } \\
\text { Sandfort (2008) } \\
\text { T2 (Pays-Bas) / } \\
\text { T4 (Etats-Unis) } \\
\\
\text { Recueil aux } \\
\text { Pays-Bas en } \\
2005 \text { et aux } \\
\text { Etats-Unis entre } \\
1997 \text { et 2002* }\end{array}$ & $\begin{array}{c}\text { Pays-Bas } \\
\text { - } 74 \text { enf de } 74 \text { couples L } \\
\text { Dont } 2 \text { couples séparés } \\
\text { AMP } \\
\text { Singletons ou issus de fratrie } \\
36 \text { G et } 38 \mathrm{~F} \\
\text { Age moy. }=11 \text { ans }(\mathrm{e}-\mathrm{t}=1,61) \\
1^{\text {ers }} \text { nés }=\text { oui } * \\
\text { Donneur }=\text { IND } *\end{array}$ & $\begin{array}{c}\text { États-Unis } \\
\text { - } 79 \text { enf de } 78 \text { familles L ( } 37 \\
\text { couples, } 34 \text { mères séparées, } 7 \\
\text { mères seules depuis la naissance } \\
\text { de l'enfant). } \\
\text { AMP } \\
\text { Singletons ou issus de fratrie } \\
38 \text { G et } 41 \mathrm{~F} \\
\text { Age moy. = } 10 \text { ans } \\
\text { une partie présents à T1 (voir } \\
\text { Gartrell et al., } 1996 \text { ) } \\
1^{\text {ers }} \text { nés = (voir Gartrell et al., } \\
1996 \text { ) } \\
\text { Donneur = 28 connus, } 31 \\
\text { anonymes, } 19 \text { pourront connaître } \\
\text { l'identité du donneur à leurs } 18 \\
\text { ans }\end{array}$ & $\begin{array}{l}\text { QE/E }=\text { DIT, } \mathrm{EH} \\
\text { QP/E }=\mathrm{CBCL}\end{array}$ \\
\hline
\end{tabular}

- DIT : les enfants de l'échantillon néerlandais ont davantage dévoilé leur situation familiale à leurs pairs que ceux de l'échantillon américain, que ce soient les filles ou les garçons. Globalement, les filles ont davantage dévoilé leur situation familiale à leurs pairs que les garçons.

- EH : les garçons $(15 \%)$ et les filles $(22 \%)$ de l'échantillon néerlandais rapportent moins d'expériences homophobes que les garçons $(37 \%)$ et filles $(46 \%)$ de l'échantillon américain.

- CBCL : les enfants de l'échantillon néerlandais présentent moins de problèmes de comportements internalisés, externalisés et totaux que ceux de l'échantillon américain.

- DIT, EH et CBCL : pour l'échantillon américain, pas de lien entre dévoilement de la situation familiale aux pairs et problèmes de comportements internalisés, externalisés et totaux; les enfants qui ont fait l'expérience de l'homophobie rapportent davantage que les autres des problèmes de comportements externalisés, d'anxiété/dépression, délinquance et comportements agressifs. Pour l'échantillon néerlandais, pas de lien entre dévoilement de la situation familiale aux pairs et problèmes de comportements internalisés, externalisés et totaux; les enfants qui ont fait l'expérience de l'homophobie rapportent davantage que les autres des problèmes de comportements internalisés et de troubles de la pensée.

\begin{tabular}{|c|c|c|c|}
\hline $\begin{array}{c}\text { Bos \& Sandfort } \\
\text { (2010) } \\
\text { T2 } \\
\text { Période de } \\
\text { recueil }=2005 \\
\end{array}$ & $\begin{array}{c}\bullet 63 \text { enf de } 63 \text { couples } \mathrm{L} \\
\text { Singletons ou issus de fratrie } \\
32 \mathrm{G} \text { et } 31 \mathrm{~F} \\
\text { Age moy. }=9,93 \text { ans }(\mathrm{e}-\mathrm{t}=1,48) \\
1^{\text {ers }} \text { nés }=\text { oui }^{*} \\
\text { Donneur }=\mathrm{IND}^{*}\end{array}$ & $\begin{array}{c}\text { - } 68 \text { enf de } 68 \text { couples } \mathrm{H} \text { (dont } \\
26 \text { n'avaient pas participé à T1) } \\
\text { Conception = naturelle* } \\
\text { Singletons ou issus de fratrie } \\
34 \mathrm{G} \text { et } 34 \mathrm{~F} \\
\text { Age moy. }=9,99 \text { ans }(\mathrm{e}-\mathrm{t}=1,32) \\
1^{\text {ers }} \text { nés }=\text { oui }^{*}\end{array}$ & $\begin{array}{l}\text { - } \mathrm{QE} / \mathrm{E}=\mathrm{MGIQ}, \mathrm{PCSC} \text {, } \\
\text { SQS }\end{array}$ \\
\hline \multicolumn{4}{|c|}{$\begin{array}{l}\text { - MGIQ : pas de différence entre enfants de mères L et ceux du groupe de comparaison quant au sentiment d'être des } \\
\text { membres typiques de leur catégorie de genre, à la satisfaction liée au genre et au sentiment de pression des pairs à se } \\
\text { conformer aux normes de genre. Les enfants de mères L ont moins le sentiment que ceux du groupe de comparaison } \\
\text { que leur genre est supérieur à l'autre genre et ils ressentent moins de pression de leurs parents à se conformer aux } \\
\text { normes de genre. }\end{array}$} \\
\hline
\end{tabular}


- PCSC : pas de différence d'estime de soi globale et de sentiment de compétence sociale entre enfants de mères L et ceux du groupe de comparaison.

- SQS : les enfants de mères L sont moins certains que les autres de s'engager à l'avenir dans des relations hétérosexuelles.

- PCSC et MGIQ / SQS : tous groupes confondus, plus les enfants ont un sentiment de typicalité de genre élevé, plus leur estime de soi globale et leur sentiment de compétence sociale sont élevés; plus les enfants sont incertains quant à leur implication future dans des relations hétérosexuelles, plus leur estime de soi globale est faible. Moins les enfants sont satisfaits de leur genre et plus ils sont incertains quant à leur implication future dans des relations hétérosexuelles et moins ils se sentent compétent socialement.

\begin{tabular}{|c|c|c|c|}
\hline $\begin{array}{c}\text { RC1/6 } \\
\text { (50) } \\
\text { Bos, van } \\
\text { Gelderen, \& } \\
\text { Gartrell (2015) } \\
\text { T3 } \\
\text { Recueil entre } \\
2010 \text { et } 2011 \\
\text { (mères L) et } \\
\text { entre 2009 et } \\
2010 \text { (groupe de } \\
\text { comparaison) }\end{array}$ & $\begin{array}{c}\bullet 51 \text { enf de } 51 \text { couples } \mathrm{L} \\
\text { Singletons ou issus de fratrie } \\
26 \mathrm{G} \text { et } 25 \mathrm{~F} \\
\text { Age moy. }=15,09 \text { ans (e-t }= \\
1,03 \text { ) } \\
1^{\text {ers }} \text { nés = oui* } \\
\text { Donneur = parmi les } 67 \\
\text { adolescents de l'échantillon } \\
\text { total, } 33 \text { avec donneur connu, } 12 \\
\text { avec donneur anonyme, } 22 \\
\text { pourront connaître l'identité du } \\
\text { donneur à leurs } 18 \text { ans* }\end{array}$ & $\begin{array}{c}\bullet 51 \text { enf de } 51 \text { couples } \mathrm{H} \\
\text { Conception }=\mathrm{IND}^{*} \\
\text { Singletons ou issus de fratrie* } \\
27 \mathrm{G} \text { et } 24 \mathrm{~F} \\
\text { Age moy. }=15,63 \text { ans }(\mathrm{e}-\mathrm{t}= \\
1,06) \\
1^{\text {ers }} \text { nés }=\text { oui } *\end{array}$ & $\begin{array}{c}\text { - } \mathrm{QE} / \mathrm{E}=\mathrm{RSES}, \mathrm{SIAS}, \\
\text { SUQ, YSR } \\
\text { - QE/E-P = ADP, FI, } \\
\text { IPPA, PCS }\end{array}$ \\
\hline
\end{tabular}

- RSES, SIAS, SUQ, YSR : les enfants de mères L ont une estime de soi plus élevée et moins de problèmes de conduite que ceux du groupe de comparaison (que ce soit pour les filles ou les garçons). Pas de différence d'anxiété sociale ni de consommation de drogue ou d'alcool entre enfants de mères L et ceux du groupe de comparaison (pour les filles comme pour les garçons).

- ADP, FI, PCS : pas de différence de perception de la qualité des relations avec les parents, de comportements de contrôle de la part des parents ni d'ouverture ou confidence aux parents entre enfants de mères L et ceux du groupe de comparaison (que ce soit pour les filles ou les garçons).

- RSES et FI / PCS / ADP : chez les enfants de couples H (mais pas chez ceux de mères L), plus la qualité des relatons avec les parents est élevée, plus l'estime de soi est élevée. Tous groupes confondus ou pris séparément, plus les parents sont perçus comme contrôlants, plus l'estime de soi des enfants est faible. Tous groupes confondus ou pris séparément, pas de lien entre degré d'ouverture aux parents et estime de soi.

- SIAS et FI / PCS / ADP : tous groupes confondus ou pris séparément, pas de lien entre (1) qualité des relations avec les parents, comportements contrôlants des parents, degré d'ouverture aux parents et (2) anxiété sociale.

- SUQ et FI / PCS / ADP : tous groupes confondus ou pris séparément, pas de lien entre (1) qualité des relations avec les parents, comportements contrôlants des parents, degré d'ouverture aux parents et (2) consommation de drogues ou d'alcool.

- YSR et FI / PCS / ADP : tous groupes confondus, les scores de problèmes de conduite sont moins élevés lorsque le degré d'ouverture aux parents est élevé, lorsque les parents sont perçus comme peu contrôlants et lorsque la qualité des relations avec les parents est élevée.

\begin{tabular}{|c|c|c|c|}
\hline $\begin{array}{l}\text { RC1/7 } \\
\quad(65) \\
\text { van Rijn-van } \\
\text { Gelderen, Bos, } \\
\text { \& Gartrell } \\
\text { (2015) } \\
\text { T3 } \\
\text { Recueil entre } \\
2010 \text { et 2011 } \\
\text { (mères L) et } \\
2003 \text { (Z-HLS) }\end{array}$ & $\begin{array}{c}\bullet 67 \text { enf de } 67 \text { couples } \mathrm{L} \\
\text { Dont } 13 \text { couples séparés } \\
\text { Singletons ou issus de fratrie } \\
31 \mathrm{G} \text { et } 36 \mathrm{~F} \\
\text { Age moy. }=16,04 \text { ans (e-t }= \\
1,32 \text { ) } \\
1^{\text {ers }} \text { nés = oui* } \\
\text { Donneur = parmi les } 67 \\
\text { adolescents de l'échantillon } \\
\text { total, } 33 \text { avec donneur connu, } 12 \\
\text { avec donneur anonyme, } 22 \\
\text { pourront connaître l'identité du } \\
\text { donneur à leurs } 18 \text { ans* }\end{array}$ & $\begin{array}{l}\text { - } 67 \text { enf de } 67 \text { couples H issus } \\
\text { de l'étude « Zuid-Holland } \\
\text { Longitudinal Study » (Z-HLS) } \\
\text { (nouvel échantillon n'ayant pas } \\
\text { participé à T1 et T2) } \\
\text { Conception = IND } \\
\text { Dont } 12 \text { couples séparés } \\
\text { Singletons vs fratrie = IND } \\
31 \text { G et } 36 \mathrm{~F} \\
\text { Age moy. }=16,03 \text { ans (e-t = } \\
1,33 \text { ) } \\
1^{\text {ers }} \text { nés }=\text { IND }\end{array}$ & $\begin{array}{l}\square \mathrm{QE} / \mathrm{E}=\mathrm{EH}, \mathrm{YSR} \\
\square \mathrm{QP} / \mathrm{E}=\mathrm{CBCL}(\mathrm{T} 1, \mathrm{~T} 3) \\
\square \mathrm{QP} / \mathrm{P}=\mathrm{CRPR}(\mathrm{T} 1)\end{array}$ \\
\hline
\end{tabular}

- CBCL (T3) et YSR : pas de différence de problèmes comportementaux internalisés et externalisés entre enfants de mères $\mathrm{L}$ et ceux de parents $\mathrm{H}$

- EH : les enfants de mères L rapportent peu d'expériences de stigmatisation (en moyenne 1,12 sur une échelle en 3 points).

- YSR et CBCL (T1, T3) / CRPR / EH : pas de lien entre l'évaluation des problèmes de comportements totaux des enfants évalués par leurs mères L à T1 et celle faite par les enfants à T3; pas de lien entre l'implication émotionnelle 
des mères L auprès de leurs enfants à T1 et les problèmes de comportements totaux évalués par leurs enfants à T3; plus les enfants de mères L rapportent d'expériences de stigmatisation, plus les scores de problèmes de comportements internalisés et externalisés sont élevés.

\begin{tabular}{|c|c|c|c|}
\hline $\begin{array}{c}\text { RC2/1 } \\
(58) \\
\text { Gartrell, } \\
\text { Rodas, Deck, } \\
\text { Peyser, \& } \\
\text { Banks (2005) } \\
\text { T4 } \\
\text { Recueil entre } \\
\text { 1997 et 2002* }\end{array}$ & $\begin{array}{c}\bullet 78 \text { enf de } 77 \text { familles L } \\
\text { Singletons ou issus de fratrie } \\
\text { Env. } 50 \% \text { de F et G* } \\
\text { Age moy. = } 10 \text { ans } \\
1^{\text {ers }} \text { nés = une partie à T1, voir } \\
\text { Gartrell et al. (66) } \\
\text { Donneur = } 27 \text { connus, } 30 \\
\text { anonymes, } 18 \text { pourront connaître } \\
\text { 1'identité du donneur à } 18 \text { ans }\end{array}$ & aucun & $\begin{array}{l}\text { - } \mathrm{QP} / \mathrm{E}=\mathrm{CBCL} \\
\text { - entretien enfants } \\
\square \text { entretien parents }\end{array}$ \\
\hline
\end{tabular}

- CBCL : les scores de compétences sociales, de problèmes internalisés, externalisés et totaux ainsi que les problèmes liés à la sexualité ne sont pas différents de ceux de la population normale de référence de l'outil (hormis pour les filles qui ont un score moyen de problèmes externalisés inférieur à celui de la population normale de référence). Pour l'ensemble des enfants, les scores de problèmes internalisés, externalisés, totaux et liés à la sexualité sont inférieurs à ceux de la population clinique de référence de l'outil.

- entretien enfants : 43\% des enfants rapportent des expériences d'homophobie et $69 \%$ de ces derniers se sont sentis en colère, bouleversés ou tristes. $57 \%$ ont dévoilé leur situation familiale à tous leurs pairs, $39 \%$ à une partie de leurs pairs, et $4 \%$ cachent ces informations à leurs camarades de classe.

- entretien mères : 74\% des enfants ont dévoilé leur conception par AMP (école ou autres environnements), 85\% ont de bons résultats scolaires, $81 \%$ ont de bonnes relations avec leurs pairs, $64 \%$ ont d'autres enfants de mères lesbiennes dans leur école, $44 \%$ ont des enseignants ou administratifs gays ou lesbiens dans leurs écoles, $47 \%$ ont des enseignements sur des thèmes LGBT.

- CBCL et entretien mères : pas de différence de problèmes ou compétences sociales selon que les enfants ont ou pourront avoir accès à l'identité du donneur et ceux qui ne le pourront pas.

\begin{tabular}{|c|c|c|c|}
\hline $\begin{array}{c}\text { RC2/2 } \\
\text { (59) } \\
\text { Bos, Gartrell, } \\
\text { Peyser, \& van } \\
\text { Balen (2008) } \\
\text { T4 } \\
\text { Recueil entre } \\
1997 \text { et } 2002^{*}\end{array}$ & $\begin{array}{c}\bullet 78 \text { enf de } 77 \text { familles L } \\
\text { Singletons ou issus de fratrie } \\
\text { Env. } 50 \% \text { de F et } \mathrm{G}^{*} \\
\text { Age moy. = } 10 \text { ans } \\
1^{\text {ers }} \text { nés }=\text { une partie à T1, voir } \\
\text { Gartrell et al. (66) } \\
\text { Donneur = } 27 \text { connus, } 30 \\
\text { anonymes, } 18 \text { pourront connaître } \\
\text { l'identité du donneur à } 18 \text { ans }\end{array}$ & aucun & $\begin{array}{l}\mathrm{QE} / \mathrm{E}=\mathrm{EH} \\
\text { - } \mathrm{QP} / \mathrm{E}=\mathrm{CBCL} \\
\square \text { entretien parents }\end{array}$ \\
\hline
\end{tabular}

- CBCL : pas de différences de problèmes internalisés, externalisés et totaux entre les enfants de mères L et ceux de la population normale de référence de l'outil.

- CBCL et EH: les enfants qui rapportent avoir fait l'expérience de l'homophobie ont des niveaux d'anxiété/dépression, de problèmes de relations sociales, d'attention, de délinquance, de comportements agressifs, internalisés et externalisés plus élevés que les autres enfants. Les enfants qui ont des enseignements sur les thèmes LGBT ont des scores de repli sur soi, de comportements agressifs, de problèmes de relations sociales, internalisés et externalisés moins élevés que les autres enfants.

- CBCL et entretien mères : pas de différences de problèmes internalisés, externalisés et totaux selon que les mères L participent ou non à la communauté lesbienne.

- CBCL et EH / entretien mères : le fait d'appartenir à une école qui délivre des enseignements sur les thèmes LGBT pourrait réduire les effets de l'homophobie sur les compétences sociales et les comportements agressifs des enfants. Les effets de l'homophobie sur les comportements délinquants sont moins forts chez les enfants dont les mères participent à la communauté lesbienne que chez les autres enfants. 


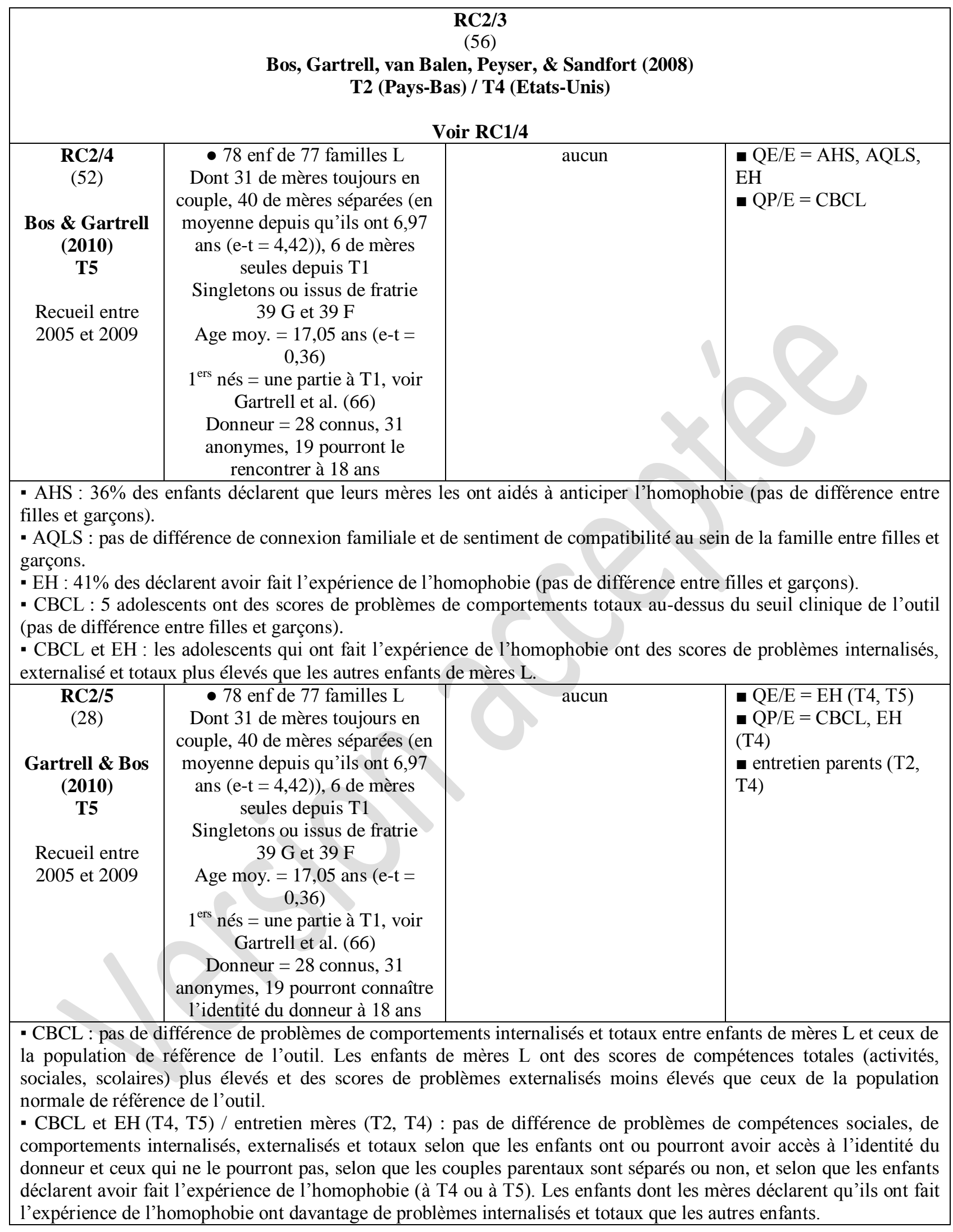




\begin{tabular}{|c|c|c|c|}
\hline $\begin{array}{c}\text { Bos \& Gartrell } \\
\text { (2011) } \\
\text { T5 }\end{array}$ & $\begin{array}{c}\text { - } 74 \text { enf de } 74 \text { ou } 75 \text { familles L } \\
\text { Pour les } 78 \text { enfants initiaux, } 31 \\
\text { sont de mères toujours en } \\
\text { couple, } 40 \text { de mères séparées (en } \\
\text { moyenne depuis qu'ils ont } 6,97 \\
\text { ans (e-t }=4,42) \text { ), } 6 \text { de mères } \\
\text { seules depuis T1 } \\
\text { Singletons ou issus de fratrie } \\
36 \mathrm{G} \text { et } 38 \mathrm{~F} \\
\text { Age moy. }=17,05 \text { ans (e-t = } \\
0,36 \text { ) } \\
1^{\text {ers }} \text { nés }=\text { une partie à T1, voir } \\
\text { Gartrell et al. (66) } \\
\text { Donneur }=26 \text { connus, } 30 \\
\text { anonymes, } 18 \text { pourront le } \\
\text { rencontrer à } 18 \text { ans }\end{array}$ & aucun & $\begin{array}{l}\text { QPP/E = CBCL (T4, } \\
\mathrm{T} 5), \mathrm{DOI}(\mathrm{T} 2)\end{array}$ \\
\hline \multicolumn{4}{|c|}{$\begin{array}{l}\text { - CBCL (T4, T5) : les scores de troubles de la pensée et de comportements délinquants sont plus élevés à T5 qu’à T4; } \\
\text { les scores de problèmes dans les relations sociales, de comportements agressifs, externalisés et totaux sont moins } \\
\text { élevés à T5 qu'à T4 (pas de différence pour les problèmes internalisés). } 6 \text { enfants }(5,4 \% \text { ) qui avaient des scores } \\
\text { « normaux » de problèmes internalisés et/ou externalisés au regard de la population de référence à T4 obtiennent à } \\
\text { T5 des scores qui dépassent les seuils cliniques fixés pour l'outil. } \\
\text { - DOI (T2) : parmi les } 48 \text { enfants qui ne connaissent pas (encore) l'identité de leur donneur, } 39,6 \% \text { indiquent que cela } \\
\text { ne les dérange pas qu'il reste anonyme, } 22,9 \% \text { regrettent de ne pas le connaître, } 37,5 \% \text { n'ont pas d'opinion sur la } \\
\text { question. } \\
\text { - CBCL (T4, T5) et DOI (T2) : pas de différence de problèmes de compétences sociales, de comportements } \\
\text { internalisés, externalisés et totaux (à T4 et à T5) selon que les enfants ont ou pourront avoir accès à l'identité du } \\
\text { donneur et ceux qui ne le pourront pas. Pas de différence de stabilité entre T4 et T5 des scores de problèmes de } \\
\text { compétences sociales, de comportements internalisés, externalisés et totaux selon que les enfants ont ou pourront } \\
\text { avoir accès à l'identité du donneur et ceux qui ne le pourront pas. }\end{array}$} \\
\hline $\begin{array}{l}\text { Recueil entre } \\
2005 \text { et } 2009 \\
\text { (mères L) et } \\
\text { entre } 2002 \text { et } \\
2003 \text { (NSFG - } \\
\text { Cycle 6) }\end{array}$ & $\begin{array}{c}\bullet 78 \text { enf de } 77 \text { familles } \mathrm{L} \\
\text { Dont } 31 \text { de mères toujours en } \\
\text { couple, } 40 \text { de mères séparées (en } \\
\text { moyenne depuis qu'ils ont } 6,97 \\
\text { ans (e-t }=4,42) \text { ), } 6 \text { de mères } \\
\text { seules depuis T1 } \\
\text { Singletons ou issus de fratrie } \\
39 \mathrm{G} \text { et } 39 \mathrm{~F} \\
\text { Age moy. }=17,05 \text { ans (e-t }= \\
0,36 \text { ) } \\
1^{\text {ers }} \text { nés = une partie à T1, voir } \\
\text { Gartrell et al. (66) } \\
\text { Donneur = 28 connus, } 31 \\
\text { anonymes, } 19 \text { pourront connaître } \\
\text { l'identité du donneur à } 18 \text { ans }\end{array}$ & \begin{tabular}{|} 
- 434 enf issus de l'étude «U.S. \\
National Survey of Family \\
Growth - Cycle 6» \\
(NSFG) \\
Conception = IND \\
Couples vs parents séparés = \\
IND \\
Singletons vs fratrie = IND \\
199 G et $235 \mathrm{~F}$ \\
Age moy. $=17$ ans \\
$1^{\text {ers }}$ nés $=$ IND
\end{tabular} & $\begin{array}{l}\text { - QE/E = victime } \\
\text { d'agression, identité } \\
\text { d'orientation sexuelle, } \\
\text { expériences sexuelles }\end{array}$ \\
\hline \multicolumn{4}{|c|}{$\begin{array}{l}\text { - victime d'agression : } 1 \text { enfant déclare avoir été agressé verbalement par sa mère non-biologique, aucun enfant ne } \\
\text { déclare d'agression physique ou sexuelle par un parent ou autre adulte proche. } \\
\text { - identité d'orientation sexuelle : } 81 \% \text { des filles et } 92 \% \text { des garçons se décrivent comme exclusivement ou de façon } \\
\text { prédominante hétérosexuel.le.s, } 0 \% \text { des filles et } 5,4 \% \text { des garçons se décrivent comme exclusivement ou de façon } \\
\text { prédominante homosexuel.le.s. Les filles }(19 \%) \text { se décrivent davantage comme non exclusivement ou de façon } \\
\text { prédominante hétéro- ou homosexuelles que les garçons }(2,7 \%) \text {. } \\
\text { - expériences sexuelles : comparés aux enfants du même âge issus de la cohorte NSFG, les garçons de mères L sont } \\
\text { moins nombreux à déclarer avoir eu un premier rapport hétérosexuel }(37,8 \% \text { contre } 58,8 \%) \text {, sans différence du côté } \\
\text { des filles }(53,8 \% \text { contre } 63,2 \%) \text {. Pas de différence entre les enfants de mères L et ceux de la cohorte NSFG } \\
\text { concernant la contraction d'une infection sexuellement transmissible, et l'implication dans une grossesse. Les filles } \\
\text { de mères L sont moins nombreuses que celles de la cohorte NSFG à avoir eu recours à une méthode contraception } \\
(47,6 \% \text { contre } 73,5 \%) \text { et plus nombreuses à avoir eu recours à une méthode de contraception d'urgence (35\% contre } \\
5,3 \%) \text {. Les filles de mères L sont plus nombreuses à déclarer avoir eu un premier rapport homosexuel ( } 15,4 \% \text { contre } \\
5,1 \%) \text {, sans différence du côté des garçons }(5,6 \% \text { contre } 6,6 \%) \text {. Pas de différence entre filles et garçons de mères L } \\
\text { concernant le fait d'avoir eu ses premiers rapports sexuels (hétéro- ou homosexuels). }\end{array}$} \\
\hline
\end{tabular}




\begin{tabular}{|c|c|c|c|}
\hline $\begin{array}{c}\text { RC2/8 } \\
(68) \\
\text { Gartrell, Bos, } \\
\text { Peyser, Deck, \& } \\
\text { Rodas (2011) } \\
\text { T5 }\end{array}$ & $\begin{array}{c}\bullet 40 \text { enf de } 40 \text { couples L } \\
\text { séparés depuis T1 (en moyenne } \\
\text { depuis qu'ils ont } 6,97 \text { ans (e-t = } \\
4,42) \text { ) } \\
\text { Singletons ou issus de fratrie } \\
21 \mathrm{G} \text { et } 19 \mathrm{~F} \\
\text { Age moy. }=17,43 \text { ans (e-t = } \\
0,49) \\
1^{\text {ers }} \text { nés = une partie à T1, voir } \\
\text { Gartrell et al. (66) } \\
\text { Donneur = IND }\end{array}$ & aucun & $\begin{array}{c}\text { - } \mathrm{QE} / \mathrm{E}=\mathrm{STPI}, \mathrm{YQOL}- \\
\mathrm{R} \\
\square \mathrm{QE} / \mathrm{E}-\mathrm{P}=\mathrm{QPCR} \\
\square \mathrm{QP} / \mathrm{E}=\mathrm{CBCL} \\
\square \mathrm{QE} / \mathrm{P}=\mathrm{QRSM} \\
\square \mathrm{QP} / \mathrm{P}=\mathrm{MPSAB}, \\
\mathrm{QRSM}\end{array}$ \\
\hline
\end{tabular}

Pour 25 enfants, la garde est partagée entre la mère biologique et la mère non-biologique et pour 10 d'entre eux la mère biologique a la garde principale; la garde est plus souvent partagée quand la mère non-biologique a adopté l'enfant.

- STPI : pas de différence d'anxiété chez les enfants selon qu'ils ont ou non été adoptés par leur mère non-biologique ni selon que la garde est partagée entre les mères ou non.

- YQOL-R : pas de différence de satisfaction de vie entre enfants adoptés et enfants non adoptés par leur mère nonbiologique.

- QPCR : comparativement aux enfants n'ayant pas été adoptés par leur mère non-biologique, les adolescents ayant été adoptés par leur mère non-biologique indiquent plus souvent être proches de leurs deux mères et passer plus de temps avec leur mère non-biologique, et rapportent une qualité de relation plus faible avec leurs mères.

- CBCL : pas de différence de problèmes de comportements internalisés, externalisés ou totaux selon qu'ils ont ou non été adoptés par leur mère non-biologique ni selon que la garde est partagée entre les mères ou non.

- CBCL et STPI / YQOL-R : comparativement aux enfants ayant des scores faibles de satisfaction de vie, ceux qui ont des scores élevés ont des scores de problèmes de comportements internalisés, totaux et d'anxiété plus faibles, que les enfants aient été adoptés ou non par leur mère non-biologique ou que la garde soit partagée ou non.

- CBCL et QRSM : chez les enfants sans adoption par la mère non-biologique, ceux qui rapportent une faible qualité de relation entre leurs mères ont des scores de problèmes de comportements externalisés et totaux plus faibles que ceux qui rapportent une qualité élevée de relation entre leurs mères (ce lien n'apparaît pas chez les enfants adoptés par leur mère non-biologique). Pour les enfants dont les mères se partagent la garde, plus les mères biologiques rapportent une communication faible avec la mère non-biologique, plus les scores de comportements internalisés, externalisés et totaux sont élevés.

\begin{tabular}{|c|c|c|c|}
\hline $\begin{array}{c}\text { Goldberg, Bos, } \\
\text { \& Gartrell } \\
(2011) \\
\text { T5 }\end{array}$ & $\begin{array}{c}\text { • } 78 \text { enf de } 77 \text { familles L } \\
\text { Dont } 31 \text { de mères toujours en } \\
\text { couple, } 40 \text { de mères séparées (en } \\
\text { moyenne depuis qu'ils ont } 6,97 \\
\text { ans (e-t }=4,42) \text { ), } 6 \text { de mères } \\
\text { seules depuis T1 } \\
\text { Singletons ou issus de fratrie } \\
39 \mathrm{G} \text { et } 39 \mathrm{~F}\end{array}$ & $\begin{array}{c}\bullet 78 \text { enf issus de la cohorte } \\
\text { «2008 Monitoring the Future: A } \\
\text { Continuing Study of the } \\
\text { Lifestyles and Values of Youth } \\
\text { (MTF) } \\
\text { Conception = IND } \\
\text { Couples vs parents séparés = } \\
\text { IND } \\
\text { Singletons vs fratrie = IND } \\
39 \text { G et } 39 \mathrm{~F} \\
\text { Age moy. }=17,05 \text { ans }(\mathrm{e}-\mathrm{t}= \\
0,36) \\
1^{\text {ers }} \text { nés }=\text { IND }\end{array}$ & $\begin{array}{l}\text { QE/E = EH, SUQ, } \\
\text { YOQL-R }\end{array}$ \\
\hline \multicolumn{4}{|c|}{$\begin{array}{l}\text { EH : } 46 \% \text { des filles et } 35,9 \% \text { des garçons déclarent avoir été victimes d'homophobie. } \\
\text { SUQ : les enfants de mères L sont plus nombreux que ceux de la cohorte MTF à avoir déjà fumé de la marijuana ou } \\
\text { du haschisch. Les garçons de mères L sont plus nombreux à avoir déjà consommé des substances hallucinogènes (pas } \\
\text { de différence concernant les filles). Pas de différences entre enfants de mères L et ceux de la cohorte MTF } \\
\text { concernant la consommation de cocaïne, barbituriques et tranquillisants. } \\
\text { SUQ et EH : chez les filles comme chez les garçons, pas de lien entre expériences de l'homophobie et } \\
\text { consommation de tabac, alcool ou marijuana/haschisch. } \\
\text { SUQ et YOQL-R : pour l'ensemble de l'échantillon, pas de lien entre satisfaction de vie et consommation de tabac, } \\
\text { alcool ou marijuana/haschisch. }\end{array}$} \\
\hline
\end{tabular}




\begin{tabular}{|c|c|c|c|}
\hline $\begin{array}{l}\text { Bos, Goldberg, } \\
\text { van Gelderen, } \\
\text { \& Gartrell } \\
(\text { (2012) } \\
\text { T5 }\end{array}$ & $\begin{array}{c}\text { - } 78 \text { enf de } 77 \text { familles } \mathrm{L} \\
\text { Dont } 31 \text { de mères toujours en } \\
\text { couple, } 40 \text { de mères séparées (en } \\
\text { moyenne depuis qu'ils ont } 6,97 \\
\text { ans (e-t }=4,42) \text { ), } 6 \text { de mères } \\
\text { seules depuis T1 } \\
\text { Singletons ou issus de fratrie } \\
39 \mathrm{G} \text { et } 39 \mathrm{~F} \\
\text { Age moy. }=17,05 \text { ans (e-t }= \\
0,36 \text { ) } \\
1^{\text {ers }} \text { nés }=\text { une partie à T1, voir } \\
\text { Gartrell et al. (66) } \\
\text { Donneur }=28 \text { connus, } 31 \\
\text { anonymes, } 19 \text { pourront connaître } \\
\text { l'identité du donneur à } 18 \text { ans }\end{array}$ & aucun & $\begin{array}{l}\text { - } \mathrm{QE} / \mathrm{E}=\mathrm{BSRI}, \mathrm{MRM} \text {, } \\
\mathrm{STPI} \\
\text { - } \mathrm{QP} / \mathrm{E}=\mathrm{CBCL}\end{array}$ \\
\hline \multicolumn{4}{|c|}{$\begin{array}{l}\text { - MRM : 47,8\% des filles et 51,4\% des garçons déclarent avoir des modèles masculins importants dans leur vie (par } \\
\text { ordre de fréquence : ami, oncle, père biologique/donneur, grand-père, enseignant, frère, autre). } \\
\text { - MRM et BSRI, STPI, CBCL : pas de lien entre le fait de disposer d'un modèle masculin important dans sa vie et (1) } \\
\text { les scores de personnalité (anxiété, peur, dépression, curiosité) des enfants (chez les filles comme chez les garçons), } \\
\text { 2) les problèmes de comportements internalisés, externalisés et totaux et 3) les scores de féminité et de masculinité } \\
\text { (chez les garçons comme chez les filles). } \\
\text { - BSRI et MRM, STPI : pas de lien entre les scores de féminité et de masculinité et l'anxiété, la peur et la dépression } \\
\text { (chez les garçons comme chez les filles). Plus les adolescents ont des scores de féminité élevés, plus ils ont des } \\
\text { scores de curiosité élevé (chez les garçons comme chez les filles, qu'ils déclarent ou non avoir des modèles } \\
\text { masculins importants dans leur vie). } \\
\text { - BSRI et MRM / CBCL (résultats valables chez les filles et les garçons) : plus les adolescents ont des scores faibles } \\
\text { de féminité, plus ils ont des scores élevés de problèmes de comportements internalisés (pas de lien avec les } \\
\text { problèmes externalisés et totaux); pas de lien entre les scores de masculinité et les problèmes de comportements } \\
\text { internalisés, externalisés et totaux. Chez les adolescents sans modèle masculin important dans leur vie, ceux qui ont } \\
\text { de faibles scores de masculinité ont des scores plus élevés de problèmes de comportements internalisés que ceux qui } \\
\text { ont des scores de masculinité élevés (cette différence n'apparaît pas chez les adolescents qui disposent d'un modèle } \\
\text { masculin important dans leur vie). }\end{array}$} \\
\hline $\begin{array}{c}\text { Recueil entre } \\
2005 \text { et 2009 } \\
\text { (mères L) et } \\
\text { entre 2006 et } \\
2008 \text { (NSFG- } \\
\text { Cycle } 7 \text { ) }\end{array}$ & $\begin{array}{c}\bullet 78 \text { enf de } 77 \text { familles } \mathrm{L} \\
\text { Dont } 31 \text { de mères toujours en } \\
\text { couple, } 40 \text { de mères séparées (en } \\
\text { moyenne depuis qu'ils ont } 6,97 \\
\text { ans (e-t }=4,42) \text { ), } 6 \text { de mères } \\
\text { seules depuis T1 } \\
\text { Singletons ou issus de fratrie } \\
39 \mathrm{G} \text { et } 39 \mathrm{~F} \\
\text { Age moy. }=17,05 \text { ans (e-t }= \\
0,36) \\
1^{\text {ers }} \text { nés }=\text { une partie à T1, voir } \\
\text { Gartrell et al. (66) } \\
\text { Donneur }=28 \text { connus, } 31 \\
\text { anonymes, } 19 \text { pourront le } \\
\text { rencontrer à } 18 \text { ans }\end{array}$ & $\begin{array}{l}\text { - } 504 \text { enf de couples H issus de } \\
\text { l'étude « U.S. National Survey } \\
\text { of Family Growth - Cycle } 7 \text { » } \\
\text { (NSFG) } \\
\text { Conception = IND } \\
\text { Couples vs parents séparés = } \\
\text { IND } \\
\text { Singletons vs fratrie = IND } \\
267 \text { G et } 237 \mathrm{~F} \\
\text { Age moy. }=17 \text { ans } \\
1^{\text {ers }} \text { nés }=\text { IND }\end{array}$ & $\begin{array}{l}\text { - QE/E = victime } \\
\text { d'agression, identité } \\
\text { d'orientation sexuelle, } \\
\text { expériences sexuelles }\end{array}$ \\
\hline \multicolumn{4}{|c|}{$\begin{array}{l}\text { Cette publication présente une réplication de Gartrell et al. (2011) [5.7] où les enfants de mères L étaient comparés à } \\
\text { des enfants de la cohorte NSFG - Cycle } 6 \text { pour lesquels les données avaient été recueillies entre } 2002 \text { et } 2003 \text {. Ici les } \\
\text { enfants de mères L sont comparés à des enfants de la cohorte NSFG - Cycle } 7 \text { pour lesquels les données ont étée } \\
\text { recueillies entre } 2006 \text { et } 2008 \text {, donc de façon plus synchrone avec le recueil de données relatif aux enfants de mères } \\
\text { L. } \\
\text { - Pour les garçons, les résultats sont les mêmes que lorsqu'ils étaient comparés à la cohorte NSFG - Cycle } 6 \text { à une } \\
\text { exception près : alors que les garçons de mères L avaient eu leur premier rapport hétérosexuel plus tardivement que } \\
\text { ceux de la cohorte NSFG - Cycle } 6 \text {, il n'y a plus de différence en comparaison avec ceux de la cohorte NSFG - } \\
\text { Cycle } 7 \text {. } \\
\text { - Pour les filles, les résultats sont les mêmes que lorsqu'elles étaient comparées à la cohorte NSFG - Cycle } 6 \text { à une } \\
\text { exception près : alors que les filles de mères L avaient davantage au recours à une contraception d'urgence que celles } \\
\text { de la cohorte NSFG - Cycle } 6 \text {, il n'y a plus de différence en comparaison avec celles de la cohorte NSFG - Cycle } 7 \text {. }\end{array}$} \\
\hline
\end{tabular}




\begin{tabular}{|c|c|c|c|}
\hline $\begin{array}{l}\text { Gartrell, Bos, } \\
\text { Peyser, Deck, \& } \\
\text { Rodas (2012) } \\
\text { T5 }\end{array}$ & $\begin{array}{c}\bullet 78 \text { enf de } 77 \text { familles } \mathrm{L} \\
\text { Dont } 31 \text { de mères toujours en } \\
\text { couple, } 40 \text { de mères séparées (en } \\
\text { moyenne depuis qu'ils ont } 6,97 \\
\text { ans }(\mathrm{e}-\mathrm{t}=4,42) \text { ), } 6 \text { de mères } \\
\text { seules depuis T1 } \\
\text { Singletons ou issus de fratrie } \\
39 \mathrm{G} \text { et } 39 \mathrm{~F} \\
\text { Age moy. }=17,05 \text { ans (e-t }= \\
0,36) \\
1^{\text {ers }} \text { nés }=\text { une partie à } \mathrm{T} 1 \text {, voir } \\
\text { Gartrell et al. (66) } \\
\text { Donneur = } 28 \text { connus, } 31 \\
\text { anonymes, } 19 \text { pourront connaître } \\
\text { l'identité du donneur à } 18 \text { ans }\end{array}$ & aucun & $\begin{array}{l}\text { - } \mathrm{QE} / \mathrm{E}=\mathrm{AEAA}, \mathrm{EAT} \text {, } \\
\mathrm{FR}, \mathrm{HP}, \mathrm{MHP}, \mathrm{OW}, \mathrm{PFF}, \\
\mathrm{RM} \\
\text { - } \mathrm{QE} / \mathrm{E}-\mathrm{P}=\mathrm{FI}\end{array}$ \\
\hline \multicolumn{4}{|c|}{ 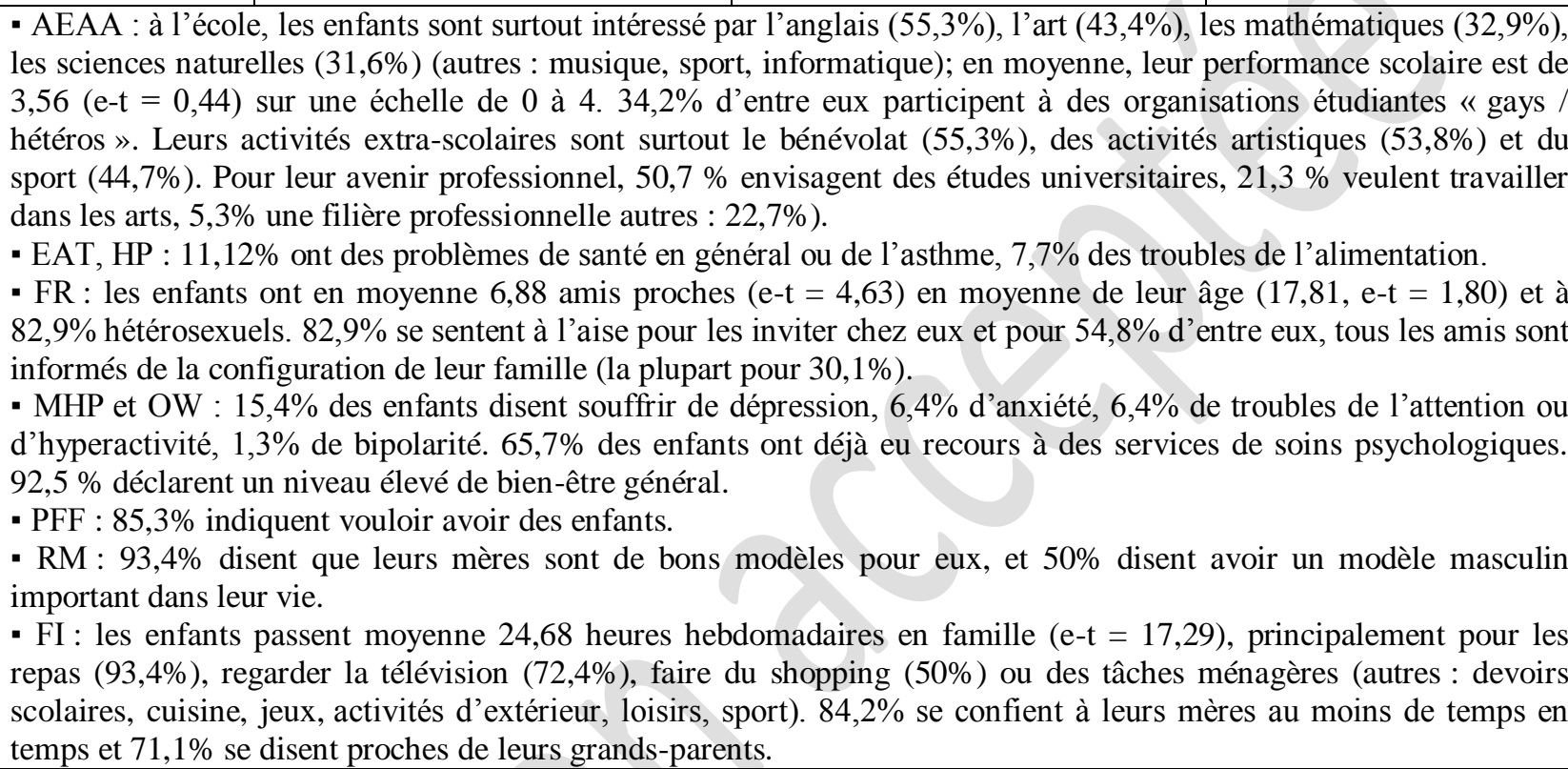 } \\
\hline $\begin{array}{c}\text { van Gelderen, } \\
\text { Bos, Gartrell, } \\
\text { Hermanns, \& } \\
\text { Perrin (2012) } \\
\text { T5 }\end{array}$ & $\begin{array}{c}\bullet 78 \text { enf de } 77 \text { familles L } \\
\text { Dont } 31 \text { de mères toujours en } \\
\text { couple, } 40 \text { de mères séparées (en } \\
\text { moyenne depuis qu'ils ont } 6,97 \\
\text { ans }(\mathrm{e}-\mathrm{t}=4,42) \text { ), } 6 \text { de mères } \\
\text { seules depuis T1 } \\
\text { Singletons ou issus de fratrie } \\
39 \mathrm{G} \text { et } 39 \mathrm{~F} \\
\text { Age moy. }=17,05 \text { ans (e-t }= \\
0,36) \\
1^{\text {ers }} \text { nés }=\text { une partie à T1, voir } \\
\text { Gartrell et al. (66) } \\
\text { Donneur }=28 \text { connus, } 31 \\
\text { anonymes, } 19 \text { pourront le } \\
\text { rencontrer à } 18 \text { ans }\end{array}$ & $\begin{array}{c}\text { - } 78 \text { enf de couples H issus de } \\
\text { l'étude « Washington Healthy } \\
\text { Youth Survey » (HYS) } \\
\text { Conception = IND } \\
\text { Couples vs parents séparés = } \\
\text { IND } \\
\text { Singletons vs fratrie = IND } \\
39 \mathrm{G} \text { et } 39 \mathrm{~F} \\
\text { Age moy. }=17,05 \text { ans } \\
1^{\text {ers }} \text { nés }=\text { IND }\end{array}$ & $\begin{array}{l}\square \mathrm{QE} / \mathrm{E}=\mathrm{EH}, \mathrm{YQOL}-\mathrm{R} \\
\mathrm{QP} / \mathrm{E}=\mathrm{DOI}(\mathrm{T} 2)\end{array}$ \\
\hline
\end{tabular}

- EH : $41 \%$ des enfants de mères L indiquent avoir fait l'expérience de l'homophobie (moqueries, stéréotypes ou exclusion), dans 28 cas de la part des camarades de classe, dans 22 cas par des enseignants, dans 21 cas par des membres de la famille élargie (autres : 10 cas).

- YQOL-R : pas de différence de satisfaction de vie entre enfants de mères L et enfants HYS, ni entre les enfants de mères L qui se sont séparées et ceux dont les mères sont toujours en couple.

- YQOL-R et DOI (T2) : pas de différence de satisfaction de vie entre les enfants qui connaissent l'identité de leur donneur, ceux qui pourront la connaître à leurs 18 ans et ceux qui ne pourront jamais la connaître (pour les filles comme pour les garçons).

- YQOL-R et EH : pas de différence de satisfaction de vie entre les enfants de mères qui ont fait l'expérience de l'homophobie et les autres enfants de mères L (pour les filles comme pour les garçons). 


\begin{tabular}{|c|c|c|c|}
\hline $\begin{array}{c}\text { van Gelderen, } \\
\text { Gartrell, Bos, } \\
\text { van Rooij, \& } \\
\text { Hermanns } \\
\left(\begin{array}{c}(2012) \\
\text { T5 }\end{array}\right.\end{array}$ & $\begin{array}{l}\text { - } 78 \text { enf de } 77 \text { familles } \mathrm{L} \\
\text { Dont } 31 \text { de mères toujours en } \\
\text { couple, } 40 \text { de mères séparées (en } \\
\text { moyenne depuis qu'ils ont } 6,97 \\
\text { ans (e-t }=4,42) \text { ), } 6 \text { de mères } \\
\text { seules depuis T1 } \\
\text { Singletons ou issus de fratrie } \\
\quad 39 \mathrm{G} \text { et } 39 \mathrm{~F} \\
\text { Age moy. }=17,05 \text { ans (e-t }= \\
0,36 \text { ) } \\
1^{\text {ers }} \text { nés }=\text { une partie à T1, voir } \\
\text { Gartrell et al. (66) } \\
\text { Donneur }=28 \text { connus, } 31 \\
\text { anonymes, } 19 \text { pourront connaître } \\
\text { l'identité du donneur à } 18 \text { ans }\end{array}$ & Aucun & - $\mathrm{QE} / \mathrm{E}=\mathrm{EH}$ \\
\hline \multicolumn{4}{|c|}{$\begin{array}{l}\text { - EH : } \\
\text { - } 50 \% \text { des enfants ont fait l'expérience de l'homophobie du fait de leur configuration familiale, les filles davantage } \\
\text { que les garçons. } \\
\text { - sources de la stigmatisation : la stigmatisation provient surtout des pairs, parfois des membres de la famille } \\
\text { étendue. } \\
\text { - contexte de la stigmatisation : la stigmatisation a surtout pris place à l'école, parfois dès l'école élémentaire. } \\
\text { - formes de la stigmatisation : les enfants disent avoir été exclus de groupes, ridiculisés ou rejetés. Les filles sont } \\
\text { plus nombreuses (50\%) à décrire plusieurs formes de stigmatisation que les garçons ( } 27 \%) \text {. } \\
\text { - stratégies de coping adaptatives et non adaptatives : } 64 \% \text { des enfants disent avoir utilisé des stratégies de coping } \\
\text { adaptatives (mise à distance des incidents, confrontation aux personnes qui les stigmatisent, recherche de soutien } \\
\text { ans l'environnement social), } 56 \% \text { des stratégies non adaptatives (non divulgation de la situation familiale, ignorer } \\
\text { les incidents, éviter les personnes concernées). Les stratégies sont davantage utilisées chez les filles }(92 \% \text { d'entre } \\
\text { elles) que chez les garçons }(80 \% \text { d'entre eux). Les filles ( } 45,8 \% \text { d'entre elles) utilisent plus fréquemment des } \\
\text { stratégies adaptatives que les garçons (13,3\% d'entre eux) et moins fréquemment des stratégies non adaptatives } \\
(16,7 \% \text { vs } 40 \%) \text {. Les garçons }(73,3 \%) \text { utilisent plus fréquemment une combinaison de stratégies adaptatives et non } \\
\text { adaptatives que les filles }(45,8 \%) \text {. }\end{array}$} \\
\hline $\begin{array}{l}\text { Bos, Gartrell, } \\
\text { \& van Gelderen } \\
(2013) \\
\text { T5 }\end{array}$ & $\begin{array}{c}\bullet 78 \text { enf de } 77 \text { familles L } \\
\text { Dont } 31 \text { de mères toujours en } \\
\text { couple, } 40 \text { de mères séparées (en } \\
\text { moyenne depuis qu'ils ont } 6,97 \\
\text { ans }(\mathrm{e}-\mathrm{t}=4,42) \text { ), } 6 \text { de mères } \\
\text { seules depuis T1 } \\
\text { Singletons ou issus de fratrie } \\
39 \mathrm{G} \text { et } 39 \mathrm{~F} \\
\text { Age moy. }=17,05 \text { ans (e-t }= \\
0,36 \text { ) } \\
1^{\text {ers }} \text { nés }=\text { une partie à T1, voir } \\
\text { Gartrell et al. (66) } \\
\text { Donneur }=28 \text { connus, } 31 \\
\text { anonymes, } 19 \text { pourront le } \\
\text { rencontrer à } 18 \text { ans }\end{array}$ & $\begin{array}{c}\text { - } 93 \text { enf issus de la population } \\
\text { «normale » de référence de la } \\
\text { base de données d'Achenbach } \\
\text { (créateur du CBCL) } \\
\text { Conception = IND } \\
\text { Couples vs parents séparés = } \\
\text { IND } \\
\text { Singletons vs fratrie = IND } \\
44 \mathrm{G} \text { et } 49 \mathrm{~F} \\
\text { Age moy. }=17 \text { ans } \\
1^{\text {ers }} \text { nés }=\text { IND }\end{array}$ & $\begin{array}{l}\text { - } \mathrm{QE} / \mathrm{E}=\mathrm{EH} \\
\text { QP/E }=\mathrm{CBCL} \text { (T4 et } \\
\mathrm{T} 5)\end{array}$ \\
\hline \multicolumn{4}{|c|}{$\begin{array}{l}\text { - CBCL (T5) : pas de différence de scores de problèmes affectifs, d'anxiété, somatiques, d'hyperactivité, } \\
\text { oppositionnels entre enfants de mères L et ceux de la base de données d'Achenbach (pour les filles comme pour les } \\
\text { garçons). Les enfants de mères L présentent plus de problèmes de conduite que ceux de la base de données } \\
\text { d'Achenbach. } \\
\text { - EH et CBCL (T5) : les enfants de mères L qui rapportent avoir été stigmatisés ont des scores de problèmes affectifs, } \\
\text { d'anxiété et de problèmes de conduite que ceux qui n'ont pas été stigmatisés. } \\
\text { - EH et CBCL (T4, T5) (échantillon restreint à } 71 \text { enfants) : pas de différence de scores au CBCL selon que les } \\
\text { enfants de mères L ont fait ou non l'expérience de l'homophobie (indépendamment du moment du recueil T4/T5); } \\
\text { entre T4 et T5, les enfants présentent moins de problèmes oppositionnels (pas de différence pour les autres scores). } \\
8,4 \% \text { des enfants de mères L présentaient des problèmes affectifs à T4 alors qu'ils n'en présentaient pas à T4, 4,2\% } \\
\text { pour les problèmes d'anxiété, 7\% pour les problèmes somatiques, } 5,6 \% \text { pour les problèmes d'hyperactivité et de } \\
\text { déficit d'attention, } 4,2 \% \text { pour les problèmes oppositionnels, et } 5,6 \% \text { pour les problèmes de conduite. Ce changement } \\
\text { dans les problèmes de conduites est davantage observé chez les enfants qui ont fait l'expérience de l'homophobie que } \\
\text { chez les autres (pas de différence pour les autres scores). }\end{array}$} \\
\hline
\end{tabular}




\begin{tabular}{|c|c|c|c|}
\hline $\begin{array}{l}\text { RC2/16 } \\
\quad(53) \\
\text { van Gelderen, } \\
\text { Gartrell, Bos, } \\
\text { \& Hermanns } \\
\quad(2013) \\
\quad \text { T5 }\end{array}$ & $\begin{array}{c}\bullet 78 \text { enf de } 77 \text { familles } \mathrm{L} \\
\text { Dont } 31 \text { de mères toujours en } \\
\text { couple, } 40 \text { de mères séparées (en } \\
\text { moyenne depuis qu'ils ont } 6,97 \\
\text { ans (e-t }=4,42) \text { ), } 6 \text { de mères } \\
\text { seules depuis T1 } \\
\text { Singletons ou issus de fratrie } \\
39 \mathrm{G} \text { et } 39 \mathrm{~F} \\
\text { Age moy. }=17,05 \text { ans (e-t }= \\
0,36 \text { ) } \\
1^{\text {ers }} \text { nés }=\text { une partie à T1, voir } \\
\text { Gartrell et al. (66) } \\
\text { Donneur }=28 \text { connus, } 31 \\
\text { anonymes, } 19 \text { pourront connaître } \\
\text { l'identité du donneur à } 18 \text { ans }\end{array}$ & aucun & $\begin{array}{l}\text { - } \mathrm{QE} / \mathrm{E}=\mathrm{BSRI}, \mathrm{EH}, \mathrm{PGF} \text {, } \\
\mathrm{STPI}, \mathrm{YQOL}-\mathrm{R} \\
\text { - QE/E-P = FC }\end{array}$ \\
\hline \multicolumn{4}{|c|}{$\begin{array}{l}\text { - EH : } 41 \% \text { des enfants de mères L indiquent avoir fait l'expérience de l'homophobie } \\
\text { - EH, STPI / YQOL-R : les enfants qui ont fait l'expérience de l'homophobie ont plus de problèmes psychologiques } \\
\text { et sont moins satisfaits de leur vie que les autres (que ce soit pour les filles ou les garçons). } \\
\text { - EH et FC / PGF / STPI : les enfants qui ont fait l'expérience de l'homophobie, ceux qui évaluent positivement leur } \\
\text { relations avec leurs parents et ceux qui se sentent intégrés dans leur groupe de pairs ont moins de problèmes } \\
\text { psychologiques que les autres. } \\
\text { - YQOL-R et BSRI / FC / PGF / STPI : les enfants qui évaluent positivement leur relations avec leurs parents et ceux } \\
\text { qui se sentent intégrés dans leur groupe de pairs sont plus satisfaits de leur vie que les autres (pas d'effet de } \\
\text { l'androgynie psychologique). }\end{array}$} \\
\hline $\begin{array}{l}\text { Baiocco, } \\
\text { Santamaria, } \\
\text { Ioverno, } \\
\text { Fontanesi, } \\
\text { Baumgartner, } \\
\text { Laghi, \& } \\
\text { Lingiardi } \\
\quad(2015)\end{array}$ & $\begin{array}{c}\bullet 20 \text { enf de } 20 \text { couples } \mathrm{L} \\
\text { Singletons ou issus de fratrie } \\
\text { Couples } L \text { et gays confondus }= \\
16 \text { G et } 24 \mathrm{~F} \\
\text { Age moy. }=3 \text { ans et } 5 \text { mois (e-t } \\
\quad=32,7 \text { mois) } \\
\quad 1^{\text {ers }} \text { nés }=85 \% \text { des enf } \\
\quad \text { Donneur = IND }\end{array}$ & $\begin{array}{c}-40 \text { enf de } 40 \text { couples } \mathrm{H} \\
\text { Conception naturelle } \\
\text { Singletons ou issus de fratrie } \\
19 \mathrm{G} \text { et } 21 \mathrm{~F} \\
\text { Age moy. }=3 \text { ans et } 10 \text { mois } \\
\text { (e-t }=17,3 \text { mois) } \\
1 \text { ers nés }=85 \% \text { des enf } \\
\text { - } 20 \text { enf de } 20 \text { couples gays } \\
\text { GPA } \\
\text { Singletons ou issus de fratrie } \\
\text { Couples L et gays confondus = } \\
16 \text { G et } 24 \mathrm{~F} \\
\text { Age moy. = } 3 \text { ans et } 6 \text { mois (e-t } \\
=16,7 \text { mois) } \\
1^{\text {ers }} \text { nés }=95 \% \text { des enf } \\
\text { Donneuse = IND }\end{array}$ & $\begin{array}{l}\text { - } \mathrm{QP} / \mathrm{E}=\mathrm{ERC}, \mathrm{SDQ} \\
\square \mathrm{QP} / \mathrm{E}-\mathrm{P}=\mathrm{FACES} \mathrm{IV} \\
\square \mathrm{QP} / \mathrm{P}=\mathrm{DAS}\end{array}$ \\
\hline \multicolumn{4}{|c|}{$\begin{array}{l}\text { - ERC : pas de différence de régulation des émotions (labilité, régulation, symptômes, problèmes comportementaux) } \\
\text { entre les enfants de couples L et ceux de couples gays et H. } \\
\text { - SDQ : pas de différence d'hyperactivité, de problèmes avec les pairs, de comportements prosociaux et de difficultés } \\
\text { totales entre les enfants de couples L et ceux de couples gays et H. } \\
\text { - FACES IV : pas de différence entre les familles de mères L et celles de pères gays (cohésion, flexibilité, } \\
\text { désengagement, enchevêtrement, rigidité, chaos, communication, satisfaction); les familles de mères L et de pères } \\
\text { gays apparaissent plus flexibles et ont davantage recours à la communication dans leur fonctionnement que les } \\
\text { familles de couples H. }\end{array}$} \\
\hline
\end{tabular}

